\title{
Supporting information: Emerging chalcogenide thin films for solar energy harvesting devices
}

\author{
Shreyash Hadke ${ }^{1,2}$, Menglin Huang ${ }^{3 *}$, Chao Chen ${ }^{4,5 *}$, Ying Fan Tay ${ }^{1,6}$, Shiyou Chen ${ }^{7}$, Jiang Tang ${ }^{4,5}$, Lydia \\ Wong ${ }^{1 *}$
}

${ }^{1}$ School of Materials Science and Engineering, Nanyang Technological University, Singapore 639798, Singapore

2Energy Research Institute @ NTU (ERI@N), Interdisciplinary Graduate Programme, Nanyang Technological University, Singapore 637553, Singapore

${ }^{3}$ Key Laboratory for Computational Physical Sciences (MOE), Key State Key Laboratory of ASIC and System and School of Microelectronics, Fudan University, Shanghai 200433, China

${ }^{4}$ School of Optical and Electronic Information, Huazhong University of Science and Technology, Wuhan, Hubei 430074, China ${ }^{5}$ Wuhan National Laboratory for Optoelectronics, Huazhong University of Science and Technology, Wuhan, Hubei 430074, China ${ }^{6}$ Institute of Materials Research and Engineering (IMRE), Agency of Science, Technology and Research (A*STAR), 2 Fusionopolis Way, Singapore 138634, Singapore

${ }^{7}$ School of microelectronics, Fudan University, Shanghai 200433, China

\section{1) EQE data presented in Figure 1 (main manuscript)}

Table S1: EQE data for Binary quasi-1D chalcogenides. Here, data for $\mathrm{Sb}_{2} \mathrm{~S}_{3}$ were obtained by tracing Figure $5 \mathrm{c}$ in ref $^{1}$, data for $\mathrm{Sb}_{2}(\mathrm{~S}, \mathrm{Se})_{3}$ were obtained from the raw data available in the supporting information of $\mathrm{ref}^{2}$, and data for $\mathrm{Sb}_{2} \mathrm{Se}_{3}$ were provided by the corresponding author(s) of ref ${ }^{3}$.

\begin{tabular}{|c|c|c|c|c|c|}
\hline \multicolumn{2}{|l|}{$\mathrm{Sb}_{2} \mathrm{~S}_{3}$} & \multicolumn{2}{|l|}{$\mathrm{Sb}_{2}(\mathrm{~S}, \mathrm{Se})_{3}$} & \multicolumn{2}{|l|}{$\mathrm{Sb}_{2} \mathrm{Se}_{3}$} \\
\hline Wavelength (nm) & EQE (\%) & Wavelength (nm) & EQE (\%) & Wavelength (nm) & EQE (\%) \\
\hline 302.1 & 2.17538 & 300.019 & 15.346 & 300 & 2.002 \\
\hline 317.48753 & 1.6709 & 309.964 & 29.548 & 310 & 2.533 \\
\hline 330.37401 & 3.18141 & 319.981 & 39.761 & 320 & 3.235 \\
\hline 334.70538 & 5.62826 & 329.995 & 48.425 & 330 & 4.313 \\
\hline 337.29432 & 8.3946 & 340.005 & 54.179 & 340 & 6.24 \\
\hline 339.3615 & 12.05232 & 350.013 & 58.347 & 350 & 8.665 \\
\hline 342.6893 & 15.21577 & 360.017 & 60.956 & 360 & 12.671 \\
\hline 344.0738 & 18.90763 & 370.018 & 65.703 & 370 & 21.575 \\
\hline 347.12118 & 22.25031 & 380.015 & 68.179 & 380 & 36.46 \\
\hline 350.19081 & 26.74006 & 390.008 & 68.765 & 390 & 48.271 \\
\hline 351.93841 & 30.36684 & 399.998 & 68.175 & 400 & 53.897 \\
\hline 354.82023 & 35.43516 & 409.984 & 68.327 & 410 & 57.167 \\
\hline 357.37236 & 39.46426 & 419.966 & 68.982 & 420 & 59.321 \\
\hline 360.82372 & 43.33254 & 430.019 & 68.552 & 430 & 61.846 \\
\hline 362.84867 & 47.07816 & 439.993 & 70.502 & 440 & 64.081 \\
\hline 365.2549 & 50.55454 & 449.963 & 70.565 & 450 & 66.181 \\
\hline 368.26743 & 54.03062 & 460.003 & 69.234 & 460 & 68.255 \\
\hline 369.53856 & 56.9926 & 469.964 & 71.177 & 470 & 70.264 \\
\hline 373.80938 & 60.23167 & 479.995 & 73.521 & 480 & 72.278 \\
\hline 375.89348 & 63.11473 & 490.021 & 77.634 & 490 & 74.273 \\
\hline 379.22859 & 65.47497 & 499.969 & 85.251 & 500 & 76.223 \\
\hline 382.00042 & 68.10232 & 509.985 & 90.785 & 510 & 78.087 \\
\hline 386.35926 & 70.30744 & 519.997 & 92.159 & 520 & 79.704 \\
\hline 391.96352 & 72.2325 & 530.004 & 92.529 & 530 & 81.061 \\
\hline 394.29889 & 73.72522 & 540.006 & 91.9 & 540 & 82.153 \\
\hline 397.82174 & 75.1261 & 550.003 & 92.178 & 550 & 82.925 \\
\hline 403.0674 & 76.67668 & 559.994 & 91.911 & 560 & 83.415 \\
\hline 407.54187 & 77.99017 & 569.98 & 91.002 & 570 & 83.818 \\
\hline 412.25034 & 79.395 & 580.034 & 90.258 & 580 & 84.141 \\
\hline 420.31006 & 80.42987 & 590.009 & 90.229 & 590 & 84.346 \\
\hline 429.33668 & 81.17989 & 599.978 & 88.887 & 600 & 84.674 \\
\hline 441.02623 & 81.69901 & 610.015 & 90.284 & 610 & 85.162 \\
\hline 450.58032 & 82.18724 & 619.972 & 89.472 & 620 & 85.614 \\
\hline 462.55886 & 82.65227 & 629.997 & 87.987 & 630 & 85.924 \\
\hline 473.06986 & 82.80592 & 640.016 & 86.884 & 640 & 86.3 \\
\hline 484.247 & 82.84604 & 650.029 & 85.045 & 650 & 86.736 \\
\hline 493.88871 & 82.80323 & 660.035 & 84.223 & 660 & 86.861 \\
\hline
\end{tabular}




\begin{tabular}{|c|c|c|c|c|c|}
\hline 504.88264 & 82.69837 & 670.035 & 82.157 & 670 & 86.898 \\
\hline 515.53773 & 82.48962 & 680.028 & 79.986 & 680 & 87.037 \\
\hline 526.03043 & 81.76972 & 690.014 & 77.527 & 690 & 87.095 \\
\hline 535.51025 & 80.85534 & 699.994 & 76.271 & 700 & 87.16 \\
\hline 546.49849 & 79.76314 & 709.967 & 75.983 & 710 & 87.218 \\
\hline 555.48017 & 78.91025 & 720.036 & 72.315 & 720 & 87.233 \\
\hline 565.27173 & 77.98261 & 730.016 & 70.81 & 730 & 87.281 \\
\hline 571.28891 & 77.2765 & 739.99 & 71.704 & 740 & 87.247 \\
\hline 579.0239 & 76.27015 & 750.031 & 71.89 & 750 & 87.125 \\
\hline 587.92782 & 75.33184 & 759.99 & 68.962 & 760 & 87.048 \\
\hline 597.30477 & 74.15732 & 770.017 & 67.962 & 770 & 86.881 \\
\hline 603.73526 & 73.54269 & 780.036 & 63.864 & 780 & 86.698 \\
\hline 613.25797 & 71.96757 & 789.973 & 58.962 & 790 & 86.581 \\
\hline 621.20045 & 70.82093 & 799.978 & 54.552 & 800 & 86.209 \\
\hline 629.25651 & 69.38327 & 809.974 & 48.519 & 810 & 85.74 \\
\hline 635.33462 & 68.0996 & 820.036 & 38.719 & 820 & 85.655 \\
\hline 641.23878 & 66.56093 & 830.017 & 33.909 & 830 & 85.513 \\
\hline 645.30179 & 64.9903 & 839.989 & 26.832 & 840 & 84.997 \\
\hline 649.99347 & 63.26539 & 850.027 & 19.371 & 850 & 84.699 \\
\hline 652.77005 & 61.80363 & 859.983 & 12.404 & 860 & 84.316 \\
\hline 656.11016 & 60.14247 & 870.005 & 7.701 & 870 & 83.487 \\
\hline 660.40107 & 58.12957 & 880.017 & 4.252 & 880 & 82.675 \\
\hline 662.71975 & 56.59644 & 890.022 & 2.211 & 890 & 81.684 \\
\hline 665.19466 & 54.92487 & 900.017 & 1.115 & 900 & 80.05 \\
\hline 666.86022 & 53.7987 & 910.004 & 0.506 & 910 & 78.785 \\
\hline 669.34684 & 52.39978 & 919.982 & 0.291 & 920 & 77.292 \\
\hline 670.16924 & 51.15647 & 930.024 & 0.106 & 930 & 74.586 \\
\hline 672.49212 & 49.76575 & 939.983 & 0.03623 & 940 & 72.068 \\
\hline 675.55238 & 47.58157 & 950.007 & 0.04599 & 950 & 69.185 \\
\hline 678.71024 & 45.16082 & 960.021 & 0.06342 & 960 & 64.933 \\
\hline 681.76115 & 43.15186 & 970.026 & 0.07749 & 970 & 60.187 \\
\hline 684.53774 & 40.69056 & 980.02 & 0.08715 & 980 & 54.791 \\
\hline 688.1049 & 38.42329 & 990.006 & 0.09124 & 990 & 48.459 \\
\hline 689.95972 & 35.93451 & 999.98 & 0.07917 & 1000 & 41.273 \\
\hline 692.50866 & 33.74876 & & & 1010 & 33.43 \\
\hline 695.04339 & 31.47318 & & & 1020 & 26.05 \\
\hline 698.29912 & 29.086 & & & 1030 & 19.053 \\
\hline 701.42768 & 26.88975 & & & 1040 & 12.891 \\
\hline 702.8508 & 25.66661 & & & 1050 & 8.299 \\
\hline 705.70217 & 23.99815 & & & 1060 & 5.02 \\
\hline 707.81756 & 22.09176 & & & 1070 & 2.869 \\
\hline 709.69426 & 20.14973 & & & 1080 & 1.656 \\
\hline 711.95221 & 18.59472 & & & 1090 & 0.767 \\
\hline 714.0231 & 17.27356 & & & 1100 & 0.581 \\
\hline 717.75561 & 15.48597 & & & -- & -- \\
\hline 720.46659 & 13.46133 & & & -- & -- \\
\hline 725.22128 & 11.9885 & & & -- & -- \\
\hline 730.0691 & 10.53789 & & & -- & -- \\
\hline 733.94449 & 9.50097 & & & -- & -- \\
\hline 739.62807 & 8.11552 & & & -- & -- \\
\hline 746.00131 & 7.3238 & & & -- & -- \\
\hline 755.22942 & 6.56896 & & & -- & -- \\
\hline 763.47815 & 6.00064 & & & -- & -- \\
\hline 772.37701 & 5.43106 & & & -- & -- \\
\hline 783.03816 & 4.83261 & & & -- & -- \\
\hline 794.83195 & 4.31736 & & & -- & -- \\
\hline 805.09579 & 3.58655 & & & -- & -- \\
\hline 815.04849 & 2.82575 & & & -- & -- \\
\hline 829.17813 & 2.05749 & & & -- & -- \\
\hline 844.57202 & 1.32693 & & & -- & -- \\
\hline 866.9129 & 0.98917 & & & -- & -- \\
\hline 889.22237 & 0.69247 & & & -- & -- \\
\hline
\end{tabular}

Table S2: EQE data for Binary quasi-2D chalcogenides. Here, data for SnS were obtained by tracing Figure $3 \mathrm{~b}$ in ref ${ }^{4}$ and data for GeSe were provided by the corresponding author(s) of ref ${ }^{5}$

\begin{tabular}{|l|l|l|l|}
\hline \multicolumn{2}{|l|}{ SnS } & GeSe & EQE (\%) \\
\hline Wavelength (nm) & EQE (\%) & Wavelength (nm) & 1.7957 \\
\hline 326.64757 & 4.17884 & 299.972 & 2.6657 \\
\hline 335.30446 & 5.95641 & 310.02 & 7.6078 \\
\hline 339.94208 & 9.67106 & 319.988 & 14.4611 \\
\hline 349.37191 & 17.89289 & 330.03 & 20.4297 \\
\hline 355.86458 & 24.03761 & 339.993 & 24.9979 \\
\hline 360.42491 & 28.07798 & 350.029 & 28.3391 \\
\hline 368.84992 & 36.00485 & 359.985 & 32.2576 \\
\hline 379.12998 & 41.73599 & 370.014 & 35.5277 \\
\hline 389.41004 & 46.12066 & 379.963 & 389.986 \\
\hline 399.14904 & 49.83759 & 400.005 & 3584 \\
\hline 412.02047 & 53.39305 & & 40.1491 \\
\hline
\end{tabular}




\begin{tabular}{|c|c|c|c|}
\hline 427.10359 & 55.84092 & 410.02 & 41.1199 \\
\hline 442.13291 & 58.38102 & 420.031 & 41.7039 \\
\hline 457.94377 & 60.64194 & 429.962 & 42.0683 \\
\hline 475.4379 & 62.61332 & 439.965 & 42.6436 \\
\hline 492.75169 & 63.8791 & 449.965 & 43.2911 \\
\hline 510.06547 & 64.9813 & 460.036 & 44.7182 \\
\hline 527.37925 & 65.95562 & 470.027 & 46.1449 \\
\hline 544.69304 & 66.77739 & 480.013 & 48.3096 \\
\hline 562.00682 & 67.8744 & 489.995 & 51.1607 \\
\hline 579.32061 & 68.98234 & 499.973 & 54.8711 \\
\hline 596.63439 & 69.91985 & 510.021 & 59.9113 \\
\hline 613.94818 & 70.5529 & 519.989 & 66.4044 \\
\hline 631.26196 & 71.03178 & 530.029 & 73.2745 \\
\hline 648.57574 & 71.40473 & 539.987 & 78.9294 \\
\hline 665.88953 & 71.66627 & 550.016 & 82.1873 \\
\hline 683.20331 & 71.83677 & 559.964 & 83.5399 \\
\hline 700.5171 & 71.75433 & 569.983 & 83.3085 \\
\hline 717.83088 & 71.68816 & 579.996 & 82.2847 \\
\hline 735.14467 & 71.36009 & 590.004 & 81.3359 \\
\hline 752.45845 & 70.91545 & 600.006 & 80.0366 \\
\hline 769.77223 & 70.03029 & 610.003 & 79.3543 \\
\hline 783.70442 & 68.08821 & 619.994 & 78.4569 \\
\hline 798.12992 & 66.07684 & 629.979 & 77.8716 \\
\hline 809.37985 & 63.56308 & 640.033 & 77.7335 \\
\hline 821.71359 & 61.90152 & 650.006 & 77.6536 \\
\hline 834.15787 & 59.74045 & 659.973 & 77.597 \\
\hline 848.73455 & 57.982 & 670.009 & 77.4385 \\
\hline 866.08016 & 56.5371 & 679.963 & 77.4484 \\
\hline 883.39394 & 55.46894 & 689.986 & 77.4667 \\
\hline 898.27298 & 53.7924 & 700.003 & 77.3918 \\
\hline 912.17811 & 51.99382 & 710.013 & 76.8234 \\
\hline 922.24058 & 50.2354 & 720.016 & 76.8297 \\
\hline 924.85106 & 49.14767 & 730.012 & 76.2172 \\
\hline 929.92474 & 47.30047 & 740.001 & 75.3749 \\
\hline 932.59574 & 46.29336 & 749.983 & 75.2054 \\
\hline 934.38298 & 45.40139 & 760.033 & 74.3967 \\
\hline 936.76596 & 44.33102 & 770.001 & 73.6694 \\
\hline 939.66374 & 42.91248 & 780.036 & 72.7147 \\
\hline 943.31915 & 41.29832 & 789.989 & 72.1799 \\
\hline 945.10638 & 40.22795 & 800.008 & 71.0801 \\
\hline 946.89362 & 39.15758 & 810.02 & 69.6649 \\
\hline 949.9438 & 37.34487 & 820.025 & 68.3195 \\
\hline 952.85106 & 35.4113 & 830.021 & 66.118 \\
\hline 955.23404 & 33.80575 & 840.009 & 65.4628 \\
\hline 957.02128 & 32.55699 & 849.989 & 64.0475 \\
\hline 959.6828 & 30.30854 & 860.035 & 61.3633 \\
\hline 962.97872 & 27.82953 & 869.998 & 58.616 \\
\hline 965.3617 & 25.51041 & 880.027 & 53.869 \\
\hline 967.14894 & 23.81566 & 889.974 & 51.1912 \\
\hline 969.96286 & 21.21011 & 899.985 & 47.4974 \\
\hline 972.51064 & 19.62339 & 909.988 & 44.0461 \\
\hline 974.89362 & 18.19623 & 919.982 & 39.7469 \\
\hline 977.2766 & 16.76908 & 929.967 & 36.9053 \\
\hline 980.24292 & 15.03019 & 940.016 & 33.3469 \\
\hline 985.02128 & 12.48761 & 949.982 & 30.3452 \\
\hline 989.69336 & 9.72891 & 960.013 & 27.9649 \\
\hline 1001.52445 & 6.93824 & 970.034 & 24.7835 \\
\hline 1015.73618 & 4.0441 & 979.971 & 21.1659 \\
\hline 1032.72533 & 2.40153 & 989.973 & 17.8471 \\
\hline 1050.03912 & 1.42123 & 999.964 & 14.4934 \\
\hline 1066.27079 & 0.88215 & 1010.019 & 10.4546 \\
\hline 1078.17401 & 0.62514 & 1019.991 & 7.046 \\
\hline \multirow[t]{18}{*}{1094.04255} & 0.62438 & 1030.025 & 4.4067 \\
\hline & & 1039.976 & 2.5458 \\
\hline & & 1049.989 & 1.4415 \\
\hline & & 1059.992 & 0.846 \\
\hline & & 1069.984 & 0.4653 \\
\hline & & 1079.965 & 0.2496 \\
\hline & & 1090.008 & 0.1398 \\
\hline & & 1099.966 & 0.2044 \\
\hline & & 1109.987 & 0.1297 \\
\hline & & 1119.996 & 0.1386 \\
\hline & & 1129.993 & 0.1647 \\
\hline & & 1139.979 & 0.1392 \\
\hline & & 1150.025 & 0.3577 \\
\hline & & 1159.987 & 0.2626 \\
\hline & & 1170.009 & 0.2817 \\
\hline & & 1180.019 & 0.1442 \\
\hline & & 1190.016 & 0.4887 \\
\hline & & 1200.018 & 0.164 \\
\hline
\end{tabular}


Table S3: EQE data for ternary quasi-2D chalcogenides. Here, data for $\mathrm{CuSbS}_{2}$ were obtained by tracing Figure 16a in ref ${ }^{6}$ and data for $\mathrm{CuSbSe}_{2}$ were obtained by tracing Figure $5 \mathrm{c}$ in ref ${ }^{7}$.

\begin{tabular}{|c|c|c|c|}
\hline \multicolumn{2}{|l|}{$\mathrm{CuSbS}_{2}$} & \multicolumn{2}{|l|}{$\mathrm{CuSbSe}_{2}$} \\
\hline Wavelength (nm) & EQE (\%) & Wavelength (nm) & EQE (\%) \\
\hline 319.72335 & 0.02289 & 302.05887 & 3.78403 \\
\hline 322.71337 & 0.01003 & 310.20553 & 3.64524 \\
\hline 328.48229 & 0.1079 & 315.72156 & 4.30773 \\
\hline 333.90388 & 0.18502 & 320.41106 & 5.24003 \\
\hline 339.67199 & 0.47108 & 325.51382 & 6.49308 \\
\hline 345.0922 & 0.8664 & 329.97874 & 7.68918 \\
\hline 349.3644 & 1.57052 & 333.80581 & 8.99919 \\
\hline 353.42704 & 2.44588 & 337.05604 & 10.1372 \\
\hline 356.58573 & 3.39065 & 342.73565 & 11.04963 \\
\hline 359.29245 & 4.36756 & 346.56272 & 11.96094 \\
\hline 361.99816 & 5.57584 & 350.38979 & 12.87225 \\
\hline 364.7043 & 6.68772 & 357.40609 & 14.01139 \\
\hline 366.507 & 7.74818 & 363.1467 & 15.20749 \\
\hline 368.30954 & 8.8472 & 368.86399 & 16.0894 \\
\hline 370.11157 & 10.06191 & 370.163 & 16.97315 \\
\hline 371.91352 & 11.2959 & 370.80085 & 18.05533 \\
\hline 373.71497 & 12.64557 & 371.4387 & 19.0236 \\
\hline 375.51575 & 14.1495 & 373.35223 & 20.50448 \\
\hline 377.31678 & 15.59558 & 375.26577 & 21.81448 \\
\hline 379.11772 & 17.06094 & 377.17931 & 23.35232 \\
\hline 380.91892 & 18.46846 & 378.455 & 24.66233 \\
\hline 382.72053 & 19.77958 & 379.09284 & 25.85842 \\
\hline 384.52248 & 21.01357 & 381.64422 & 27.3393 \\
\hline 386.32452 & 22.22827 & 383.55776 & 29.16192 \\
\hline 388.1273 & 23.26945 & 383.98209 & 28.36196 \\
\hline 389.93001 & 24.32991 & 384.83345 & 30.07323 \\
\hline 391.7333 & 25.25541 & 385.4713 & 31.09845 \\
\hline 393.53659 & 26.1809 & 386.10914 & 32.23759 \\
\hline 395.33946 & 27.2028 & 388.02268 & 33.60456 \\
\hline 398.04487 & 28.48177 & 389.29837 & 34.91456 \\
\hline 401.20367 & 29.40084 & 390.57406 & 35.99675 \\
\hline 404.81443 & 30.28777 & 391.84975 & 36.96501 \\
\hline 407.97278 & 31.30967 & 393.76329 & 38.27502 \\
\hline 410.67973 & 32.23516 & 394.40113 & 39.47111 \\
\hline 413.83816 & 33.23778 & 396.95252 & 40.43938 \\
\hline 417.90019 & 34.25582 & 398.86605 & 41.46461 \\
\hline 422.86645 & 35.14018 & 400.47457 & 42.61177 \\
\hline 427.38059 & 36.09138 & 402.53613 & 43.41886 \\
\hline 430.99119 & 37.01687 & 407.80456 & 44.45266 \\
\hline 434.60179 & 37.94236 & 411.69862 & 45.54355 \\
\hline 438.21272 & 38.79073 & 417.42517 & 46.46399 \\
\hline 442.27535 & 39.66995 & 423.15173 & 47.5225 \\
\hline 445.88597 & 40.5903 & 427.96203 & 48.43783 \\
\hline 449.04443 & 41.58649 & 432.77234 & 49.22532 \\
\hline 453.5586 & 42.53126 & 436.6664 & 50.26167 \\
\hline 458.97695 & 43.35392 & 441.11726 & 50.87543 \\
\hline 464.39542 & 44.15087 & 447.79228 & 50.11506 \\
\hline 469.81483 & 44.7293 & 456.62753 & 49.60226 \\
\hline 474.32942 & 45.57767 & 463.10672 & 49.69869 \\
\hline 477.48741 & 46.68312 & 467.0335 & 50.64543 \\
\hline 480.19413 & 47.66003 & 470.5676 & 51.67301 \\
\hline 483.35257 & 48.66265 & 473.90537 & 52.74053 \\
\hline 486.511 & 49.66526 & 477.43947 & 53.82753 \\
\hline 489.21817 & 50.53934 & 482.24977 & 54.70195 \\
\hline 492.3758 & 51.72834 & 487.6491 & 55.53692 \\
\hline 494.85599 & 52.84664 & 492.09945 & 56.6434 \\
\hline 497.33655 & 53.88139 & 496.22257 & 57.86384 \\
\hline 499.68145 & 54.94057 & 499.62578 & 58.96448 \\
\hline 501.84516 & 56.10514 & 504.23974 & 60.06609 \\
\hline 503.64845 & 57.03064 & 507.283 & 60.78783 \\
\hline 505.45132 & 58.05253 & 515.23473 & 60.92516 \\
\hline 507.25436 & 59.03587 & 523.48097 & 60.53653 \\
\hline 509.05757 & 59.98064 & 531.04002 & 59.87944 \\
\hline 510.86027 & 61.0411 & 533.44517 & 59.15076 \\
\hline 512.66356 & 61.9666 & 536.19392 & 58.56781 \\
\hline 514.4666 & 62.94993 & 539.97345 & 57.6443 \\
\hline 516.26972 & 63.91399 & 544.70271 & 56.86001 \\
\hline 518.07276 & 64.89732 & 549.84179 & 56.36539 \\
\hline 519.87605 & 65.82281 & 558.31606 & 56.77279 \\
\hline 521.67934 & 66.74831 & 563.8946 & 55.81584 \\
\hline 523.48263 & 67.6738 & 567.55959 & 54.78007 \\
\hline 525.73666 & 68.84994 & 573.30199 & 53.90424 \\
\hline 527.9911 & 69.92968 & 580.05933 & 53.38776 \\
\hline 531.60048 & 71.13668 & 584.29698 & 52.723 \\
\hline
\end{tabular}




\begin{tabular}{|c|c|c|c|}
\hline 536.56675 & 72.01847 & 592.19963 & 52.2321 \\
\hline 541.98572 & 72.69973 & 600.44586 & 51.93551 \\
\hline 547.40703 & 72.84113 & 608.98661 & 51.37302 \\
\hline 552.82985 & 72.63546 & 615.66214 & 50.6279 \\
\hline 558.25301 & 72.35267 & 620.03068 & 49.92332 \\
\hline 563.67789 & 71.67141 & 626.65712 & 49.23409 \\
\hline 568.65045 & 71.10583 & 632.46876 & 48.77124 \\
\hline 572.26953 & 70.07751 & 640.20452 & 48.29611 \\
\hline 575.43681 & 69.04275 & 646.02924 & 47.47989 \\
\hline 579.50696 & 68.19053 & 652.67204 & 47.19012 \\
\hline 584.48081 & 67.32674 & 660.82011 & 47.00749 \\
\hline 589.90687 & 66.37554 & 665.13957 & 46.25361 \\
\hline 595.33142 & 65.7714 & 670.44072 & 45.56108 \\
\hline 600.7553 & 65.3215 & 674.76018 & 44.78966 \\
\hline 606.17929 & 64.8459 & 680.84669 & 44.30266 \\
\hline 611.602 & 64.66595 & 688.17668 & 44.90947 \\
\hline 616.12245 & 64.16464 & 694.45299 & 45.59264 \\
\hline 620.64496 & 63.18773 & 700.31698 & 45.6356 \\
\hline 626.06851 & 62.81496 & 704.21104 & 44.92992 \\
\hline 631.49066 & 62.76355 & 707.74514 & 44.10591 \\
\hline 634.61384 & 62.92068 & 713.14446 & 43.56971 \\
\hline 636.91314 & 62.63501 & 722.07789 & 43.37284 \\
\hline 641.88698 & 61.77378 & 729.36206 & 43.97266 \\
\hline 644.3489 & 61.26019 & 736.60697 & 43.79909 \\
\hline 646.85982 & 61.14394 & 740.92643 & 43.11534 \\
\hline 652.28286 & 60.88685 & 747.1602 & 42.65183 \\
\hline 657.70601 & 60.60407 & 756.24088 & 42.51924 \\
\hline 660.33423 & 60.37064 & 763.00367 & 42.9254 \\
\hline 662.22733 & 59.90352 & 767.4322 & 43.95494 \\
\hline 664.50769 & 59.36249 & 773.15876 & 43.6413 \\
\hline 666.40016 & 59.4472 & 777.74 & 42.72086 \\
\hline 669.37084 & 59.5404 & 785.98624 & 42.47541 \\
\hline 672.17068 & 59.17726 & 794.23248 & 42.36291 \\
\hline 674.58503 & 58.88807 & 802.47872 & 41.93337 \\
\hline 677.59434 & 58.77879 & 807.97621 & 41.07941 \\
\hline 680.14457 & 58.71016 & 813.4737 & 40.32771 \\
\hline 681.66385 & 58.07182 & 818.97119 & 39.48397 \\
\hline 685.73421 & 57.17203 & 824.46869 & 38.855 \\
\hline 691.15703 & 56.96637 & 830.65337 & 38.40245 \\
\hline 694.39614 & 57.04967 & 837.21981 & 38.93682 \\
\hline 695.22436 & 56.7607 & 845.08428 & 39.51977 \\
\hline 697.94013 & 55.65525 & 853.33052 & 39.28454 \\
\hline 699.61624 & 55.03336 & 861.57676 & 38.9675 \\
\hline 702.01065 & 54.71691 & 869.823 & 38.72205 \\
\hline 707.43514 & 54.12562 & 874.04429 & 38.07482 \\
\hline 712.85847 & 53.80427 & 878.75643 & 37.27593 \\
\hline 714.90853 & 53.72869 & 882.87954 & 36.46185 \\
\hline 716.32694 & 52.77594 & 887.59168 & 35.7781 \\
\hline 717.83699 & 51.86331 & 895.93609 & 35.44937 \\
\hline 721.00341 & 51.02779 & 904.18233 & 35.3471 \\
\hline 724.30132 & 50.88213 & 912.52674 & 35.03298 \\
\hline 725.07187 & 50.56505 & 917.92606 & 34.49824 \\
\hline 726.88426 & 49.39533 & 926.85949 & 34.35251 \\
\hline 728.69659 & 48.23846 & 932.35698 & 34.19143 \\
\hline 731.86348 & 47.29369 & 934.12403 & 33.32358 \\
\hline 735.03004 & 46.42604 & 937.85447 & 32.41191 \\
\hline 737.74547 & 45.39771 & 941.97759 & 31.7001 \\
\hline 740.46123 & 44.29227 & 946.7879 & 30.93153 \\
\hline 743.17711 & 43.16111 & 952.28539 & 30.16449 \\
\hline 745.29033 & 42.08137 & 957.09569 & 29.22104 \\
\hline 746.80049 & 41.14302 & 961.21881 & 28.50514 \\
\hline 748.61241 & 40.08256 & 966.02912 & 27.70998 \\
\hline 750.42424 & 39.04138 & 970.83943 & 26.80744 \\
\hline 752.23682 & 37.82667 & 974.76621 & 25.81102 \\
\hline 754.04957 & 36.5734 & 978.57028 & 24.87962 \\
\hline 755.86255 & 35.26872 & 983.89597 & 24.35292 \\
\hline 756.771 & 34.16327 & 991.45502 & 24.04202 \\
\hline 758.58412 & 32.82645 & 992.8294 & 23.40384 \\
\hline 759.49246 & 31.7467 & 996.95252 & 22.38676 \\
\hline 761.30443 & 30.67339 & 1001.41923 & 21.42029 \\
\hline 764.02011 & 29.58722 & 1007.35848 & 20.80557 \\
\hline 765.83216 & 28.49463 & 1013.17012 & 20.3357 \\
\hline 767.64349 & 27.56914 & 1021.00405 & 19.83253 \\
\hline 769.45482 & 26.64364 & 1023.06561 & 19.12072 \\
\hline 771.26648 & 25.64103 & 1028.36676 & 18.40365 \\
\hline 773.07857 & 24.542 & 1032.68622 & 17.53756 \\
\hline 774.89073 & 23.4237 & 1036.12215 & 16.77461 \\
\hline 776.70273 & 22.34396 & 1039.55808 & 16.07712 \\
\hline 778.51414 & 21.39919 & 1043.6812 & 15.30395 \\
\hline 780.32508 & 20.56367 & 1045.44825 & 14.52026 \\
\hline 782.58812 & 19.66389 & 1049.96405 & 13.68748 \\
\hline 785.33873 & 18.41276 & 1054.08717 & 12.75827 \\
\hline
\end{tabular}




\begin{tabular}{|c|c|c|c|}
\hline 788.09113 & 17.15735 & 1056.05056 & 11.88604 \\
\hline 790.01707 & 16.0721 & 1060.17368 & 11.05764 \\
\hline 792.82432 & 14.55265 & 1063.51144 & 10.18103 \\
\hline 795.26507 & 13.72531 & 1065.67117 & 9.24131 \\
\hline 797.98073 & 12.64557 & 1067.04555 & 8.50086 \\
\hline 801.14832 & 11.54013 & 1071.85585 & 7.74405 \\
\hline 803.41253 & 10.37041 & 1077.25518 & 6.93757 \\
\hline 806.58018 & 9.2521 & 1080.78928 & 6.25907 \\
\hline 809.29516 & 8.32661 & 1085.59958 & 5.47363 \\
\hline 812.0107 & 7.27258 & 1090.60623 & 4.74604 \\
\hline 814.7258 & 6.32138 & 1096.36551 & 3.85774 \\
\hline 817.44078 & 5.39589 & 1102.19023 & 3.15061 \\
\hline 821.51158 & 4.39327 & 1111.58582 & 2.17482 \\
\hline 826.93607 & 3.80198 & 1122.02047 & 1.56686 \\
\hline 832.36141 & 3.01788 & 1128.89234 & 1.36232 \\
\hline 837.78696 & 2.18237 & 1138.76683 & 0.91131 \\
\hline 843.21118 & 1.65535 & 1151.56949 & 0.62596 \\
\hline 848.63511 & 1.19261 & 1157.06699 & 0.62596 \\
\hline 854.05909 & 0.71993 & 1168.10773 & 0.51261 \\
\hline 857.34378 & 0.6307 & 1177.67541 & 0.51261 \\
\hline 862.19349 & 0.37304 & 1187.88094 & 0.51261 \\
\hline 867.61603 & 0.23147 & & \\
\hline 873.03855 & 0.09505 & & \\
\hline 878.4609 & -0.00282 & & \\
\hline 883.88283 & -0.00282 & & \\
\hline 889.30475 & -0.00282 & & \\
\hline 894.72668 & -0.00282 & & \\
\hline 900.1485 & 0.02084 & & \\
\hline 905.57053 & -0.00282 & & \\
\hline 910.99246 & -0.00282 & & \\
\hline 916.41439 & -0.00282 & & \\
\hline 921.83631 & -0.00282 & & \\
\hline 927.25824 & -0.00282 & & \\
\hline 932.68017 & -0.00282 & & \\
\hline 938.10209 & -0.00282 & & \\
\hline 943.52402 & -0.00282 & & \\
\hline 949.46244 & -0.03166 & & \\
\hline
\end{tabular}

Table S4: EQE for ternary 3D chalcogenides. Here, data for $\mathrm{Cu}_{2} \mathrm{SnS}_{3}$ were provided by corresponding author(s) of $\mathrm{ref}^{8}$, data for $\mathrm{Cu}_{2}(\mathrm{Sn}, \mathrm{Ge}) \mathrm{S}_{3}$ were obtained by tracing Figure $5 \mathrm{~b}$ in ref ${ }^{9}$, data for $\mathrm{Cu}_{2} \mathrm{GeS}_{3}$ were obtained by tracing Figure $8 \mathrm{~b}$ in $\mathrm{ref}^{10}$, and data for $\mathrm{AgBiS}_{2}$ were provided by the corresponding author(s) of ref ${ }^{11}$.

\begin{tabular}{|c|c|c|c|c|c|c|c|}
\hline \multicolumn{2}{|l|}{$\mathrm{Cu}_{2} \mathrm{SnS}_{3}$} & \multicolumn{2}{|c|}{$\mathrm{Cu}_{2}(\mathrm{Sn}, \mathrm{Ge}) \mathrm{S}_{3}$} & \multicolumn{2}{|l|}{$\mathrm{Cu}_{2} \mathrm{GeS}_{3}$} & \multicolumn{2}{|l|}{$\mathrm{AgBiS}_{2}$} \\
\hline $\begin{array}{l}\text { Wavelength } \\
(\mathrm{nm})\end{array}$ & EQE (\%) & $\begin{array}{l}\text { Wavelength } \\
(\mathrm{nm})\end{array}$ & EQE (\%) & $\begin{array}{l}\text { Wavelength } \\
(\mathrm{nm})\end{array}$ & EQE (\%) & $\begin{array}{l}\text { Wavelength } \\
(\mathrm{nm})\end{array}$ & EQE (\%) \\
\hline 300 & -0.07565 & 307.67205 & 2.12151 & 302.40136 & 0.95406 & 299.972 & 19.8775 \\
\hline 310 & 0.26124 & 325.20722 & 3.91741 & 311.60719 & 1.42083 & 310.02 & 25.3444 \\
\hline 320 & 0.76332 & 341.13201 & 5.97833 & 321.00481 & 2.14602 & 319.988 & 28.7795 \\
\hline 330 & 3.9885 & 351.50997 & 7.90393 & 328.29276 & 3.24516 & 330.03 & 33.6021 \\
\hline 340 & 7.6842 & 360.9734 & 10.06822 & 332.89568 & 4.44556 & 339.993 & 38.2283 \\
\hline 350 & 14.224 & 371.55017 & 12.25751 & 336.34786 & 5.5004 & 350.029 & 41.8457 \\
\hline 360 & 22.837 & 384.07529 & 14.08105 & 340.51926 & 6.73719 & 359.985 & 46.8668 \\
\hline 370 & 33.802 & 396.60041 & 15.80985 & 344.01939 & 8.06716 & 370.014 & 54.9489 \\
\hline 380 & 48.544 & 410.37804 & 17.75179 & 347.47158 & 9.52284 & 379.963 & 60.6264 \\
\hline 390 & 59.117 & 426.2432 & 19.82399 & 350.3484 & 10.86951 & 389.986 & 64.6049 \\
\hline 400 & 64.986 & 439.18582 & 21.6932 & 352.64986 & 12.01928 & 400.005 & 66.6322 \\
\hline 410 & 68.395 & 451.43261 & 23.5671 & 354.95131 & 13.1796 & 410.02 & 68.5904 \\
\hline 420 & 69.469 & 460.65746 & 25.60077 & 357.71854 & 14.45745 & 420.031 & 70.1696 \\
\hline 430 & 70.799 & 469.24611 & 27.57993 & 360.18439 & 15.67805 & 429.962 & 71.3767 \\
\hline 440 & 70.775 & 476.76119 & 29.64028 & 362.23926 & 16.95854 & 439.965 & 71.9173 \\
\hline 450 & 71.588 & 482.18874 & 31.66512 & 364.54072 & 18.35093 & 449.965 & 72.0011 \\
\hline 460 & 73.775 & 486.78128 & 33.92677 & 366.84218 & 19.75122 & 460.036 & 71.2564 \\
\hline 470 & 75.907 & 491.79133 & 36.13937 & 369.14364 & 21.17525 & 470.027 & 70.1622 \\
\hline 480 & 77.446 & 496.80138 & 39.00661 & 371.19851 & 22.53146 & 480.013 & 69.3254 \\
\hline 490 & 78.9 & 502.08977 & 42.10504 & 373.45887 & 23.9521 & 489.995 & 68.4799 \\
\hline 500 & 80.735 & 506.32047 & 44.84429 & 375.76033 & 25.44733 & 499.973 & 67.4906 \\
\hline 510 & 82.856 & 510.16151 & 47.33487 & 378.15768 & 27.02606 & 510.021 & 66.8652 \\
\hline 520 & 84.899 & 512.66654 & 49.52548 & 380.40434 & 28.54402 & 519.989 & 66.7596 \\
\hline 530 & 85.878 & 515.17156 & 51.63715 & 383.52775 & 29.85654 & 530.029 & 66.3031 \\
\hline 540 & 86.475 & 518.63088 & 53.86722 & 386.97994 & 31.11179 & 539.987 & 66.4065 \\
\hline 550 & 86.605 & 522.20949 & 56.6076 & 390.92529 & 32.33239 & 550.016 & 66.5995 \\
\hline 560 & 86.566 & 524.35665 & 58.91154 & 394.54187 & 33.45352 & 559.964 & 67.365 \\
\hline 570 & 86.698 & 529.92337 & 61.3666 & 399.06259 & 34.60066 & 569.983 & 67.9903 \\
\hline 580 & 85.906 & 535.31613 & 64.47983 & 404.24087 & 35.75095 & 579.996 & 68.1334 \\
\hline 590 & 85.458 & 540.10252 & 66.97704 & 409.99452 & 36.92815 & 590.004 & 68.6083 \\
\hline 600 & 85.352 & 547.97545 & 69.41293 & 415.74816 & 38.08003 & 600.006 & 69.2764 \\
\hline
\end{tabular}




\begin{tabular}{|c|c|c|c|c|c|c|c|}
\hline 610 & 85.188 & 556.92197 & 71.61411 & 420.92644 & 39.1971 & 610.003 & 69.3132 \\
\hline 620 & 85.394 & 566.94206 & 73.59116 & 425.97686 & 40.35214 & 619.994 & 69.6287 \\
\hline 630 & 85.366 & 585.72975 & 75.32926 & 430.13227 & 41.47027 & 629.979 & 69.49 \\
\hline 640 & 85.024 & 605.76994 & 76.17294 & 437.61201 & 42.42263 & 640.033 & 69.7977 \\
\hline 650 & 85.1 & 625.81013 & 76.51338 & 445.09175 & 43.41117 & 650.006 & 69.1775 \\
\hline 660 & 85.266 & 645.85033 & 76.42457 & 452.57149 & 44.466 & 659.973 & 69.1867 \\
\hline 670 & 85.167 & 665.89052 & 75.59569 & 460.77043 & 45.39162 & 670.009 & 68.1468 \\
\hline 680 & 84.881 & 685.93072 & 74.73721 & 466.38024 & 46.44065 & 679.963 & 67.0716 \\
\hline 690 & 84.141 & 705.97091 & 73.93793 & 471.99004 & 47.59095 & 689.986 & 66.3489 \\
\hline 700 & 83.146 & 726.01111 & 73.13866 & 478.01538 & 48.61502 & 700.003 & 65.2435 \\
\hline 710 & 82.476 & 746.0513 & 72.32458 & 483.51331 & 49.68392 & 710.013 & 64.1484 \\
\hline 720 & 81.657 & 766.0915 & 71.5401 & 488.24409 & 50.77919 & 720.016 & 62.859 \\
\hline 730 & 80.448 & 786.13169 & 70.84444 & 493.35844 & 51.80062 & 730.012 & 61.4759 \\
\hline 740 & 79.492 & 806.17189 & 70.69642 & 498.72851 & 52.78513 & 740.001 & 59.9177 \\
\hline 750 & 78.358 & 826.21208 & 70.14877 & 504.22643 & 53.79777 & 749.983 & 58.034 \\
\hline 760 & 76.623 & 846.25227 & 69.52711 & 510.31716 & 54.64995 & 760.033 & 56.3361 \\
\hline 770 & 76.213 & 866.29247 & 69.70473 & 516.05337 & 53.89622 & 770.001 & 54.7333 \\
\hline 780 & 75.643 & 886.33266 & 69.18668 & 520.33664 & 52.82732 & 780.036 & 52.4068 \\
\hline 790 & 74.649 & 906.37286 & 68.62423 & 527.82916 & 51.5355 & 789.989 & 50.89 \\
\hline 800 & 74.21 & 926.41305 & 67.94336 & 532.62935 & 50.43335 & 800.008 & 48.8824 \\
\hline 810 & 73.559 & 945.20073 & 66.28349 & 537.06787 & 49.42071 & 810.02 & 47.1488 \\
\hline 820 & 73.118 & 954.14725 & 64.42274 & 540.68445 & 48.36286 & 820.025 & 44.7996 \\
\hline 830 & 72.378 & 961.48339 & 62.68886 & 545.20517 & 47.32197 & 830.021 & 43.3917 \\
\hline 840 & 72.283 & 966.49344 & 60.79428 & 549.72589 & 46.211 & 840.009 & 41.301 \\
\hline 850 & 71.693 & 970.25098 & 58.91154 & 552.68491 & 45.28877 & 849.989 & 39.8734 \\
\hline 860 & 71.568 & 977.76605 & 56.61042 & 556.1371 & 44.30777 & 860.035 & 37.7854 \\
\hline 870 & 71.233 & 984.20754 & 54.10405 & 560.16465 & 43.11317 & 869.998 & 35.9521 \\
\hline 880 & 70.98 & 992.7962 & 51.53847 & 565.21507 & 41.94846 & 880.027 & 33.8597 \\
\hline 890 & 70.651 & 996.55373 & 49.56495 & 570.45728 & 40.91472 & 889.974 & 32.0018 \\
\hline 900 & 70.252 & 1000.31127 & 47.85193 & 576.68583 & 39.90007 & 899.985 & 30.193 \\
\hline 910 & 69.858 & 1005.32132 & 46.21786 & 583.54537 & 38.60879 & 909.988 & 28.1581 \\
\hline 920 & 69.777 & 1009.91386 & 44.29565 & 590.0836 & 37.59903 & 919.982 & 26.0848 \\
\hline 930 & 69.19 & 1016.05714 & 42.11068 & 599.28943 & 36.70505 & 929.967 & 24.1487 \\
\hline 940 & 68.71 & 1021.60398 & 40.05258 & 607.34454 & 35.70032 & 940.016 & 21.8964 \\
\hline 950 & 68.321 & 1030.6499 & 37.487 & 615.39964 & 34.71141 & 949.982 & 19.6939 \\
\hline 960 & 67.723 & 1041.64417 & 34.96583 & 624.60547 & 33.70668 & 960.013 & 17.9384 \\
\hline 970 & 67.331 & 1052.63844 & 33.00053 & 633.8113 & 32.93929 & 970.034 & 15.8241 \\
\hline 980 & 67.063 & 1060.43185 & 31.15595 & 643.01713 & 32.30639 & 979.971 & 14.0481 \\
\hline 990 & 66.49 & 1067.94693 & 29.31663 & 652.22297 & 31.48362 & 989.973 & 12.0817 \\
\hline 1000 & 65.483 & 1075.462 & 27.16549 & 661.4288 & 30.89819 & 999.964 & 10.5938 \\
\hline 1010 & 64.559 & 1081.72456 & 25.00448 & 670.63463 & 30.10706 & 1010.019 & 8.8522 \\
\hline 1020 & 63.909 & 1087.98712 & 22.93821 & 679.84046 & 29.57701 & 1019.991 & 7.3427 \\
\hline 1030 & 62.981 & 1092.16216 & 20.77128 & 689.04629 & 29.30802 & 1030.025 & 6.0816 \\
\hline 1040 & 62.37 & 1095.50219 & 18.95959 & 698.25212 & 29.12606 & 1039.976 & 4.9672 \\
\hline 1050 & 61.3 & 1100.92975 & 16.92292 & 707.45796 & 28.53272 & 1049.989 & 4.0479 \\
\hline 1060 & 60.302 & 1105.52229 & 14.64942 & 716.66379 & 28.08969 & 1059.992 & 3.2682 \\
\hline 1070 & 59.404 & 1110.11484 & 12.32461 & 725.86962 & 27.78906 & 1069.984 & 2.4563 \\
\hline 1080 & 58.207 & 1115.12488 & 9.97612 & 735.07545 & 27.37767 & 1079.965 & 2.0871 \\
\hline 1090 & 56.979 & 1121.98388 & 7.94058 & 744.28128 & 26.75269 & 1090.008 & 1.6766 \\
\hline 1100 & 55.857 & 1130.57253 & 5.5357 & 753.48711 & 25.98529 & 1099.966 & 1.2919 \\
\hline 1110 & 54.45 & 1138.08761 & 3.38456 & 760.96685 & 25.09923 & 1109.987 & 0.9451 \\
\hline 1120 & 52.63 & 1148.94271 & 1.52945 & 764.9944 & 24.03121 & 1119.996 & 0.8915 \\
\hline 1130 & 49.699 & 1169.40041 & 0.44895 & 769.51513 & 22.93833 & 1129.993 & 0.685 \\
\hline 1140 & 47.145 & 1189.44061 & 0.21213 & 773.1317 & 21.88952 & 1139.979 & 0.7579 \\
\hline 1150 & 44.472 & 1209.4808 & 0.16772 & 777.57023 & 20.77742 & 1150.025 & 0.3314 \\
\hline 1160 & 41.927 & 1229.521 & 0.22693 & 780.52925 & 19.79552 & 1159.987 & 0.1567 \\
\hline 1170 & 38.987 & 1249.56119 & 0.21213 & 783.98143 & 18.83351 & 1169.993 & 0.3763 \\
\hline 1180 & 35.139 & 1269.60138 & 0.15292 & 787.43362 & 17.72594 & & \\
\hline 1190 & 31.446 & 1289.64158 & 0.13812 & 791.46117 & 16.56298 & & \\
\hline 1200 & 27.888 & 1309.68177 & 0.22693 & 797.07097 & 15.47123 & & \\
\hline 1210 & 25.399 & 1329.72197 & 0.16772 & 804.11919 & 14.49023 & & \\
\hline 1220 & 23.525 & 1349.76216 & 0.13812 & 813.32502 & 13.53297 & & \\
\hline 1230 & 21.962 & 1369.80236 & 0.10852 & 821.38012 & 12.64163 & & \\
\hline 1240 & 20.992 & 1389.84255 & 0.18252 & 826.27072 & 11.63426 & & \\
\hline 1250 & 20.235 & 1401.11516 & 0.1382 & 832.50384 & 11.04883 & & \\
\hline 1260 & 19.466 & & & 840.94252 & 10.75611 & & \\
\hline 1270 & 18.806 & & & 848.27841 & 9.59316 & & \\
\hline 1280 & 18.368 & & & 852.44981 & 8.50668 & & \\
\hline 1290 & 17.55 & & & 855.32663 & 7.4413 & & \\
\hline 1300 & 15.982 & & & 858.77882 & 6.27043 & & \\
\hline 1310 & 13.452 & & & 862.231 & 5.13121 & & \\
\hline 1320 & 10.471 & & & 866.25855 & 4.09483 & & \\
\hline 1330 & 7.7696 & & & 871.82041 & 3.05318 & & \\
\hline 1340 & 5.6924 & & & 881.21803 & 1.99835 & & \\
\hline 1350 & 4.0847 & & & 890.42386 & 1.43665 & & \\
\hline 1360 & 2.9212 & & & 899.62969 & 1.049 & & \\
\hline 1370 & 2.1229 & & & 908.83553 & 0.74837 & & \\
\hline 1380 & 1.6217 & & & 918.04136 & 0.4873 & & \\
\hline 1390 & 1.1002 & & & 927.24719 & 0.34489 & & \\
\hline 1400 & 0.88532 & & & 936.45302 & 0.24205 & & \\
\hline 1410 & 0.77014 & & & 945.65885 & 0.1392 & & \\
\hline 1420 & 0.45617 & & & 954.86468 & 0.04427 & & \\
\hline
\end{tabular}




\begin{tabular}{|l|l|l|l|l|l|l|}
\hline 1430 & 0.40698 & & & 964.07052 & 0.03635 & \\
\hline 1440 & 0.35809 & & & 973.27635 & 0.03635 & \\
\hline 1450 & 0.28152 & & & 982.48218 & 0.03635 & \\
\hline & -- & & & 991.68801 & 0.03635 & \\
\hline & -- & & & 998.01702 & & 0.05745 \\
\hline
\end{tabular}

Table S5: EQE data for kesterites. Here, data for $\mathrm{Cu}_{2} \mathrm{ZnSnS}_{4}$ were provided by the corresponding author(s) of ref $^{12}$, data for $\mathrm{Cu}_{2} \mathrm{ZnSn}(\mathrm{S}, \mathrm{Se})_{4}$-IBM were provided by the corresponding author(s) of ref ${ }^{13}$, data for $\mathrm{Cu}_{2} \mathrm{ZnSn}(\mathrm{S}, \mathrm{Se})_{4}$-DGIST were provided by the corresponding author(s) of ref $^{14}$, and data for $\mathrm{Cu}_{2} \mathrm{ZnSnSe}_{4}$ were obtained by tracing Figure $2 \mathrm{c}$ in ref $^{15}$.

\begin{tabular}{|c|c|c|c|c|c|c|c|}
\hline \multicolumn{2}{|l|}{$\mathrm{Cu}_{2} \mathrm{ZnSnS}_{4}$} & \multicolumn{2}{|c|}{$\mathrm{Cu}_{2} \mathrm{ZnSn}(\mathrm{S}, \mathrm{Se})_{4}-\mathrm{IBM}$} & \multicolumn{2}{|c|}{$\mathrm{Cu}_{2} \mathrm{ZnSn}(\mathrm{S}, \mathrm{Se})_{4}-\mathrm{DGIST}$} & \multicolumn{2}{|l|}{$\mathrm{Cu}_{2} \mathrm{ZnSnSe}{ }_{4}$} \\
\hline $\begin{array}{l}\text { Wavelength } \\
(\mathrm{nm})\end{array}$ & EQE (\%) & $\begin{array}{l}\text { Wavelength } \\
(\mathrm{nm})\end{array}$ & EQE (\%) & $\begin{array}{l}\text { Wavelength } \\
(\mathrm{nm})\end{array}$ & EQE (\%) & $\begin{array}{l}\text { Wavelength } \\
(\mathrm{nm})\end{array}$ & EQE (\%) \\
\hline 284.5 & 0.28 & 310 & 30.17 & 309.998 & 1.342 & 319.07154 & 0.15876 \\
\hline 286 & 0.24 & 320 & 34.3 & 320.002 & 1.7489 & 330.92668 & 0.91617 \\
\hline 287 & 0.26 & 330 & 37.94 & 329.994 & 4.1279 & 338.83011 & 2.35002 \\
\hline 288 & 0.32 & 340 & 42.83 & 340.011 & 9.4893 & 342.25493 & 3.82775 \\
\hline 289 & 0.36 & 350 & 45.92 & 350.015 & 15.6496 & 345.94319 & 5.50102 \\
\hline 290 & 0.48 & 360 & 47.76 & 360.006 & 21.2349 & 348.70939 & 7.06634 \\
\hline 291 & 0.57 & 370 & 53.07 & 369.983 & 29.5192 & 350.02663 & 8.70341 \\
\hline 292 & 0.76 & 380 & 60.54 & 379.983 & 41.1198 & 353.31972 & 10.5734 \\
\hline 293 & 1 & 390 & 65.94 & 390.007 & 51.663 & 354.41742 & 12.56293 \\
\hline 294 & 1.13 & 400 & 67.93 & 400.015 & 58.787 & 357.43609 & 14.90192 \\
\hline 295 & 1.41 & 410 & 69.91 & 410.008 & 63.1206 & 359.74126 & 17.69858 \\
\hline 296 & 1.85 & 420 & 71.18 & 419.985 & 65.7674 & 362.35221 & 20.4019 \\
\hline 297.5 & 1.95 & 430 & 74.16 & 429.984 & 67.5359 & 364.95532 & 23.61302 \\
\hline 298.5 & 2.35 & 440 & 75.95 & 440.002 & 69.7538 & 365.17486 & 24.88636 \\
\hline 320.5 & 2.23 & 450 & 77.77 & 450.004 & 71.3996 & 365.17486 & 21.95309 \\
\hline 321.5 & 2.46 & 460 & 78.76 & 459.988 & 73.2836 & 367.62116 & 26.53796 \\
\hline 322.5 & 2.65 & 470 & 79.25 & 469.991 & 74.7638 & 368.46796 & 28.29688 \\
\hline 323.5 & 2.82 & 480 & 80.23 & 479.997 & 77.7512 & 369.91692 & 29.77022 \\
\hline 324.5 & 2.85 & 490 & 81.79 & 490.001 & 81.2358 & 371.10243 & 31.29134 \\
\hline 325.5 & 2.93 & 500 & 83.14 & 499.986 & 85.0906 & 371.3847 & 32.70143 \\
\hline 326.5 & 3.05 & 510 & 84.81 & 509.988 & 89.4539 & 373.95645 & 34.59502 \\
\hline 327.5 & 3.17 & 520 & 86.38 & 520.006 & 92.7133 & 374.01917 & 36.44333 \\
\hline 328.5 & 3.37 & 530 & 87.5 & 530.003 & 94.5476 & 376.37138 & 38.61386 \\
\hline 330 & 3.44 & 540 & 88.66 & 540.015 & 95.6009 & 376.81046 & 40.48407 \\
\hline 331 & 3.58 & 550 & 89.29 & 550.005 & 95.6704 & 379.2254 & 42.18927 \\
\hline 332 & 3.72 & 560 & 89.13 & 560.009 & 96.6045 & 379.66448 & 44.01116 \\
\hline 333 & 3.87 & 570 & 88.69 & 569.99 & 96.1193 & 380.3231 & 46.032 \\
\hline 334 & 4.02 & 580 & 88.5 & 579.984 & 96.0867 & 381.77206 & 47.5872 \\
\hline 335 & 4.16 & 590 & 89 & 589.99 & 96.1994 & 383.61619 & 49.20376 \\
\hline 336 & 4.32 & 600 & 89.01 & 600.008 & 95.8383 & 384.74525 & 51.08935 \\
\hline 337 & 4.48 & 610 & 88.39 & 610 & 96.1272 & 387.34836 & 53.72843 \\
\hline 338 & 4.65 & 620 & 89.18 & 620.003 & 96.5066 & 387.5679 & 54.89945 \\
\hline 339 & 4.83 & 630 & 88.68 & 630.016 & 95.9699 & 389.54376 & 56.44551 \\
\hline 340.5 & 5.01 & 640 & 88.27 & 640.001 & 96.3607 & 392.27232 & 58.9199 \\
\hline 343.5 & 5.2 & 650 & 88.02 & 649.995 & 96.1408 & 393.49547 & 60.76552 \\
\hline 344.5 & 5.4 & 660 & 88.1 & 659.997 & 96.5605 & 396.12995 & 62.40249 \\
\hline 345.5 & 5.58 & 670 & 87.62 & 670.005 & 95.7579 & 399.61122 & 64.6046 \\
\hline 346.5 & 5.77 & 680 & 86.77 & 679.985 & 95.804 & 404.50382 & 66.98201 \\
\hline 347.5 & 5.97 & 690 & 88.24 & 690.005 & 95.6379 & 409.30233 & 69.14115 \\
\hline 348.5 & 6.15 & 700 & 87.71 & 699.994 & 94.9568 & 414.47719 & 71.09365 \\
\hline 349.5 & 6.34 & 710 & 88.49 & 709.988 & 94.6496 & 418.52299 & 72.76949 \\
\hline 350.5 & 6.56 & 720 & 87.88 & 719.985 & 94.0656 & 424.45056 & 74.46076 \\
\hline 351.5 & 6.76 & 730 & 88.26 & 729.985 & 93.4532 & 430.37813 & 76.28165 \\
\hline 352.5 & 6.97 & 740 & 88.43 & 739.987 & 92.7348 & 437.62294 & 77.50214 \\
\hline 354 & 7.2 & 750 & 88.25 & 749.989 & 92.3149 & 445.52636 & 79.18936 \\
\hline 355 & 7.4 & 760 & 88.21 & 759.992 & 91.9895 & 454.74703 & 80.74784 \\
\hline 356 & 7.64 & 770 & 87.53 & 769.994 & 91.4712 & 464.62631 & 82.36718 \\
\hline 357 & 7.86 & 780 & 89 & 779.994 & 90.7116 & 473.84697 & 83.85014 \\
\hline 358 & 8.13 & 790 & 86.92 & 789.992 & 90.2395 & 481.7504 & 85.50943 \\
\hline 359 & 8.31 & 800 & 86.85 & 799.987 & 90.302 & 489.65383 & 86.79353 \\
\hline 360 & 8.85 & 810 & 86.06 & 810.012 & 89.9237 & 497.55725 & 88.31639 \\
\hline 361 & 8.64 & 820 & 86.22 & 819.998 & 89.4279 & 508.75377 & 89.66741 \\
\hline 362 & 8.8 & 830 & 86.13 & 830.012 & 89.2702 & 522.58477 & 90.55188 \\
\hline 363 & 9.36 & 840 & 84.04 & 839.985 & 89.2413 & 536.41576 & 91.51325 \\
\hline 364 & 10.07 & 850 & 84.52 & 849.985 & 89.8383 & 550.90538 & 91.5183 \\
\hline 365.5 & 10.88 & 860 & 83.93 & 860.008 & 88.9456 & 565.39499 & 91.66599 \\
\hline 366.5 & 11.61 & 870 & 82.23 & 869.989 & 88.5352 & 579.88461 & 91.57183 \\
\hline 367.5 & 12.24 & 880 & 82.24 & 879.992 & 88.194 & 594.37422 & 91.06216 \\
\hline 368.5 & 12.9 & 890 & 80.78 & 890.015 & 88.0963 & 608.86384 & 90.73854 \\
\hline 369.5 & 13.56 & 900 & 80.69 & 899.994 & 87.4234 & 623.35345 & 90.2847 \\
\hline
\end{tabular}




\begin{tabular}{|c|c|c|c|c|c|c|c|}
\hline 370.5 & 14.36 & 910 & 79.99 & 909.99 & 87.5763 & 637.84307 & 90.15332 \\
\hline 371.5 & 15.25 & 920 & 77.25 & 920.004 & 86.8344 & 652.33268 & 89.87311 \\
\hline 372.5 & 16.21 & 930 & 78.85 & 930.002 & 86.3211 & 666.8223 & 89.82856 \\
\hline 373.5 & 17.28 & 940 & 76.26 & 940.015 & 85.463 & 681.31191 & 89.7654 \\
\hline 374.5 & 18.4 & 950 & 73.5 & 950.009 & 84.4641 & 695.80153 & 89.88651 \\
\hline 376 & 19.54 & 960 & 73.74 & 960.014 & 83.966 & 710.29114 & 89.67628 \\
\hline 377 & 20.72 & 970 & 72.72 & 969.999 & 83.0248 & 724.78076 & 89.22864 \\
\hline 378 & 21.9 & 980 & 72 & 979.993 & 81.8849 & 739.27037 & 89.02284 \\
\hline 379 & 23.05 & 990 & 71.89 & 989.995 & 80.5341 & 753.75999 & 88.63101 \\
\hline 380 & 24.11 & 1000 & 67.32 & 1000.003 & 78.5083 & 768.2496 & 88.64227 \\
\hline 382 & 26.1 & 1020 & 60.57 & 1019.999 & 72.9345 & 785.34351 & 88.56852 \\
\hline 383 & 27.11 & 1030 & 58.14 & 1030.015 & 69.4757 & 799.97692 & 87.40631 \\
\hline 384 & 28 & 1040 & 55.39 & 1040.001 & 66.5254 & 805.79088 & -- \\
\hline 385 & 28.84 & 1050 & 51.74 & 1050.014 & 62.275 & 809.88638 & 87.76394 \\
\hline 386 & 29.56 & 1060 & 49.94 & 1059.994 & 58.0977 & 815.57252 & 86.95323 \\
\hline 387.5 & 30.33 & 1070 & 45.49 & 1069.998 & 54.3319 & 820.15267 & 87.80676 \\
\hline 388.5 & 31.01 & 1080 & 39.25 & 1079.994 & 49.4124 & 829.47701 & 86.33054 \\
\hline 389.5 & 31.67 & 1090 & 36.38 & 1090.01 & 43.7235 & 835.93842 & 86.52833 \\
\hline 390.5 & 32.32 & 1100 & 32.39 & 1100.014 & 38.1304 & 847.57145 & 85.06647 \\
\hline 392.5 & 33.45 & 1120 & 24.31 & 1120.008 & 28.1393 & 863.74933 & 84.51853 \\
\hline 393.5 & 33.97 & 1130 & 19.23 & 1129.994 & 24.0547 & 876.26309 & 83.13927 \\
\hline 394.5 & 34.49 & 1140 & 17.36 & 1139.989 & 19.7517 & 890.75271 & 82.1521 \\
\hline 395.5 & 34.98 & 1150 & 14.61 & 1149.988 & 16.2343 & 905.24232 & 80.74944 \\
\hline 396.5 & 35.42 & 1160 & 12.44 & 1159.991 & 12.5003 & 919.73194 & 79.03548 \\
\hline 398 & 35.85 & 1170 & 8.333 & 1169.994 & 9.2141 & 926.41221 & 79.35433 \\
\hline 399 & 36.28 & 1180 & 6.681 & 1179.995 & 6.6123 & 932.2457 & 78.23728 \\
\hline 400 & 36.72 & 1190 & 5.762 & 1189.99 & 5.11 & 944.75945 & 76.7774 \\
\hline 401 & 37.1 & 1200 & 4.872 & 1200.004 & 3.6791 & 957.93183 & 75.37209 \\
\hline 402 & 37.47 & 1210 & 4.601 & & & 969.12835 & 73.80887 \\
\hline 403 & 37.88 & 1220 & 3.387 & & & 979.00763 & 72.1697 \\
\hline 404 & 38.26 & 1230 & 3.104 & & & 990.71898 & 70.15047 \\
\hline 405 & 38.63 & 1240 & 4.829 & & & 995.1145 & 70.05099 \\
\hline 406 & 38.99 & 1250 & 1.607 & & & 999.16741 & 69.13847 \\
\hline 407 & 39.39 & 1260 & 1.598 & & & 1006.10687 & 68.72184 \\
\hline 408.5 & 39.78 & 1270 & 1.583 & & & 1011.93858 & 67.57608 \\
\hline 409.5 & 40.14 & 1280 & 1.724 & & & 1025.11095 & 66.30926 \\
\hline 410.5 & 40.48 & 1290 & 1.425 & & & 1036.33588 & 65.96803 \\
\hline 411.5 & 40.83 & 1300 & 0.87 & & & 1037.48092 & 65.02833 \\
\hline 415.5 & 42.29 & 1340 & 2.27 & & & 1074.76478 & 58.82463 \\
\hline 416.5 & 42.62 & 1350 & 2.245 & & & 1081.97171 & 56.5272 \\
\hline 417.5 & 42.97 & 1360 & 1.367 & & & 1089.6556 & 54.99986 \\
\hline 418.5 & 43.3 & 1370 & 1.126 & & & 1098.21765 & 53.35389 \\
\hline 420 & 43.64 & 1380 & 2.752 & & & 1104.54039 & 51.0568 \\
\hline 421 & 43.97 & 1390 & 1.437 & & & 1113.36588 & 49.35753 \\
\hline 422 & 44.29 & 1400 & 3.602 & & & 1119.16173 & 47.36518 \\
\hline 423 & 44.62 & & & & & 1123.24516 & 45.70892 \\
\hline 424 & 44.95 & & & & & 1129.83135 & 44.13092 \\
\hline 425 & 45.27 & & & & & 1133.38789 & 42.58896 \\
\hline 426 & 45.6 & & & & & 1139.05201 & 41.17986 \\
\hline 427 & 45.93 & & & & & 1143.00373 & 39.46564 \\
\hline 428 & 46.26 & & & & & 1148.66785 & 37.62166 \\
\hline 429 & 46.59 & & & & & 1154.85887 & 35.99423 \\
\hline 430.5 & 46.91 & & & & & 1157.58743 & 34.58829 \\
\hline 431.5 & 47.22 & & & & & 1162.76229 & 32.8159 \\
\hline 432.5 & 47.53 & & & & & 1168.68986 & 31.16688 \\
\hline 433.5 & 47.84 & & & & & 1172.4534 & 29.43001 \\
\hline 434.5 & 48.17 & & & & & 1177.25191 & 27.59342 \\
\hline 435.5 & 48.49 & & & & & 1182.52086 & 25.59769 \\
\hline 436.5 & 48.82 & & & & & 1187.39464 & 23.83905 \\
\hline 437.5 & 49.15 & & & & & 1193.2783 & 21.8952 \\
\hline 438.5 & 49.46 & & & & & 1198.47328 & 21.17343 \\
\hline 439.5 & 49.78 & & & & & 1201.62081 & 20.19521 \\
\hline 440.5 & 50.09 & & & & & 1204.25528 & 18.83069 \\
\hline 442 & 50.37 & & & & & 1210.05113 & 17.15971 \\
\hline 443 & 50.65 & & & & & 1214.13456 & 15.39696 \\
\hline 444 & 50.94 & & & & & 1219.4976 & 13.56325 \\
\hline 445 & 51.22 & & & & & 1225.9897 & 11.6527 \\
\hline 446 & 51.49 & & & & & 1231.91727 & 10.00141 \\
\hline 447 & 51.75 & & & & & 1238.50346 & 8.45922 \\
\hline 448 & 52.03 & & & & & 1245.74827 & 6.86467 \\
\hline 449 & 52.3 & & & & & 1253.6517 & 5.40878 \\
\hline 450 & 52.57 & & & & & 1263.53098 & 3.72156 \\
\hline 451 & 52.86 & & & & & 1276.70336 & 2.44579 \\
\hline 452.5 & 53.14 & & & & & 1291.19297 & 1.60745 \\
\hline 453.5 & 53.41 & & & & & 1305.68258 & 0.88074 \\
\hline
\end{tabular}




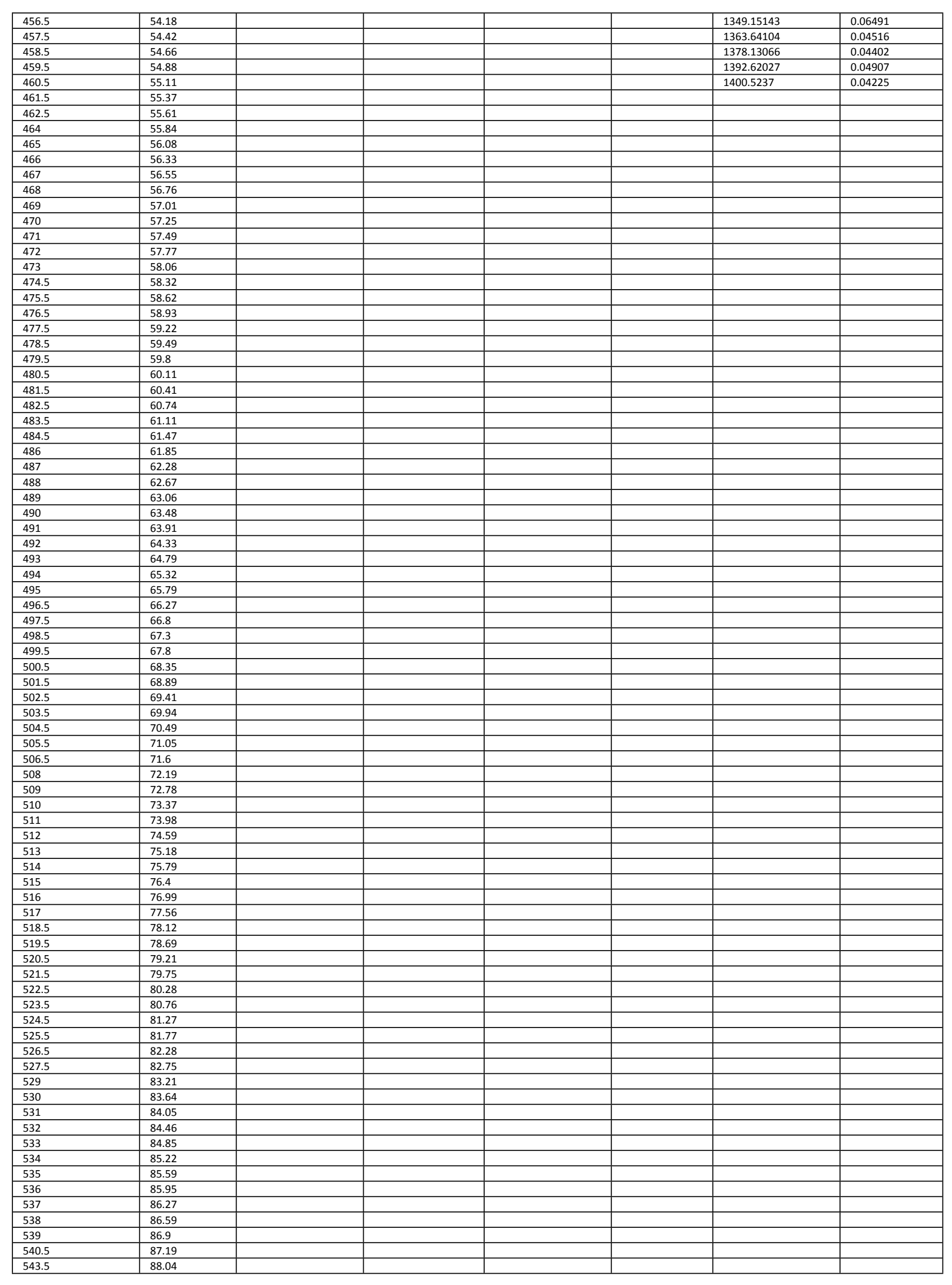




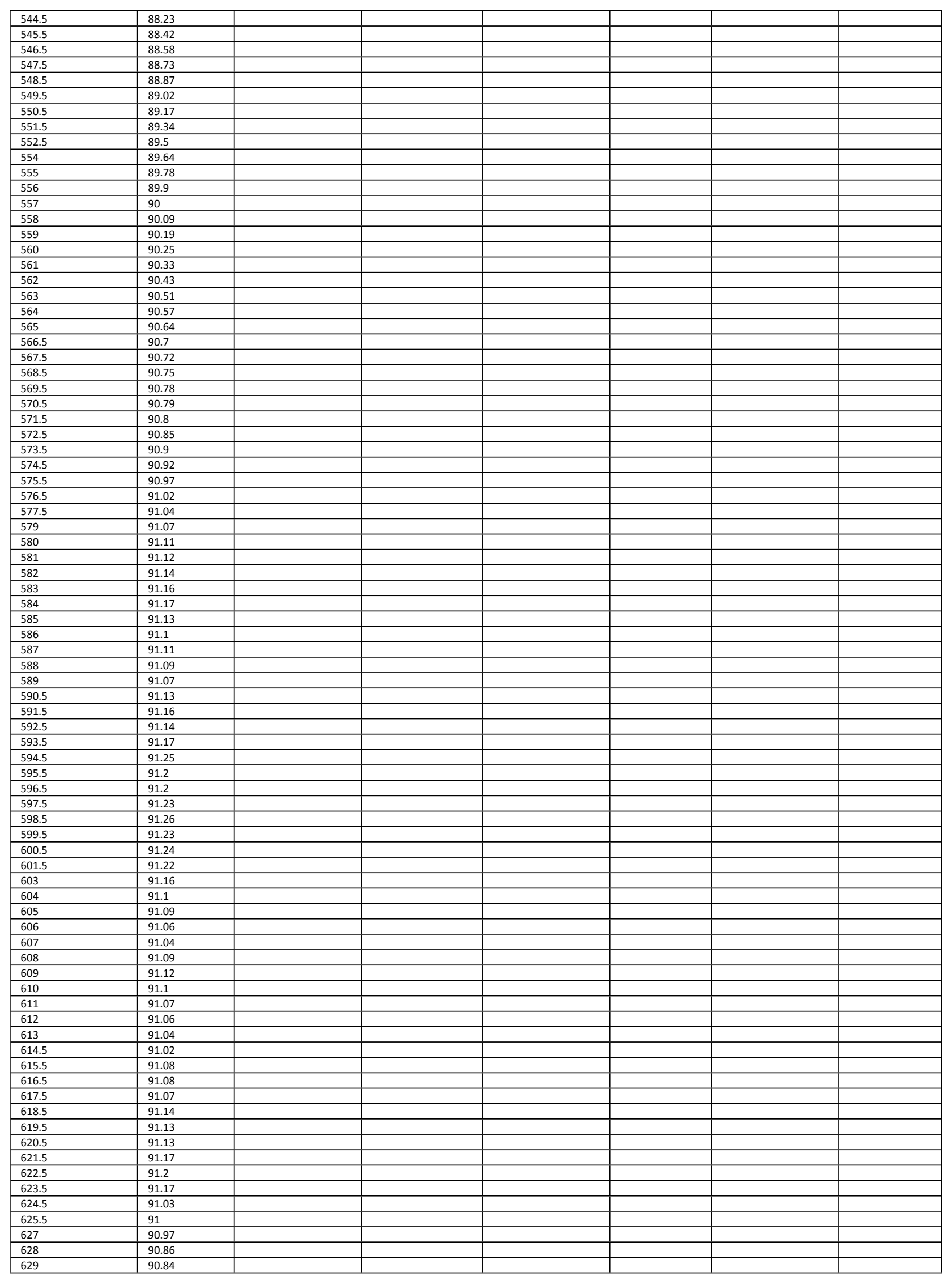




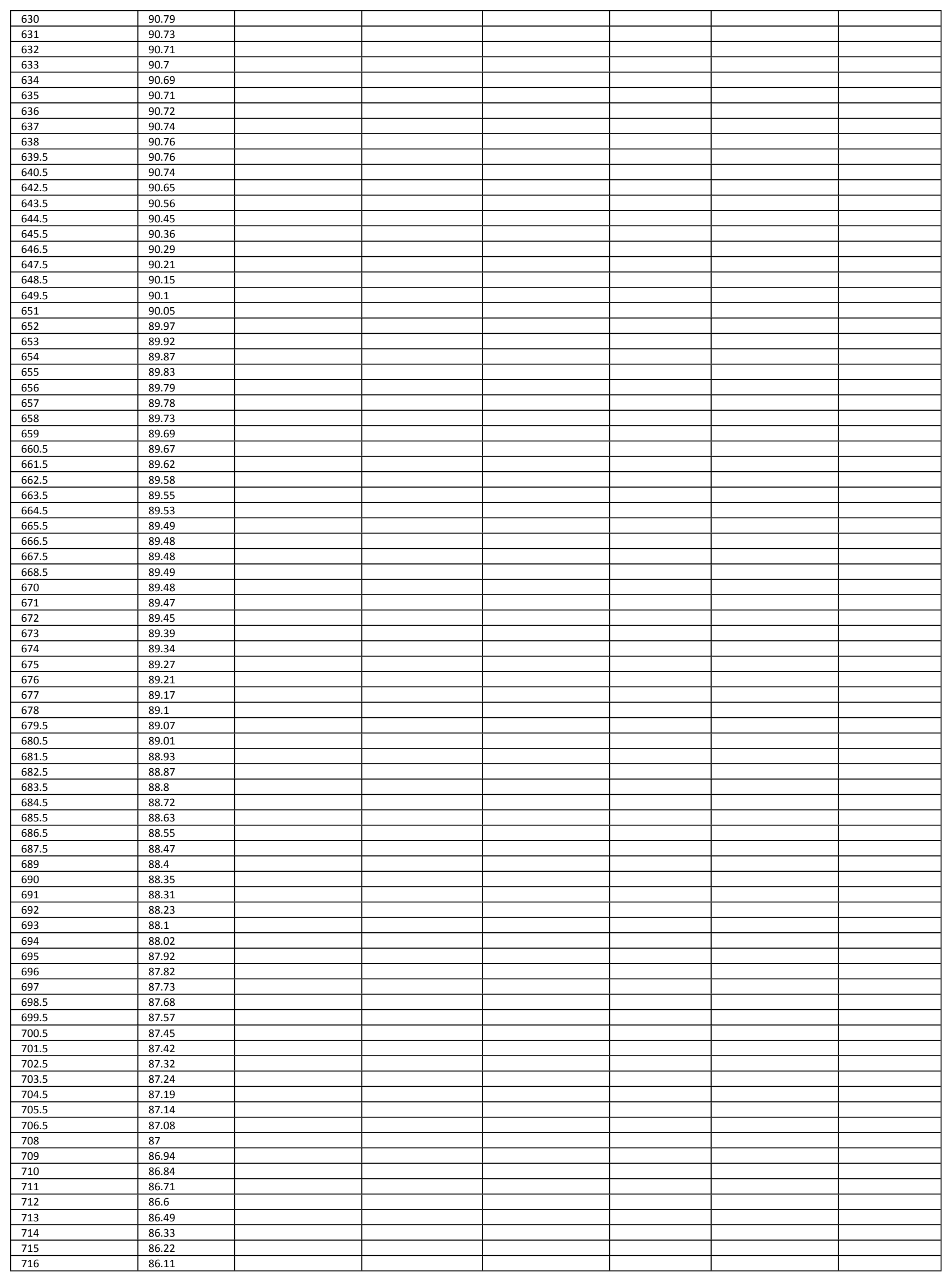




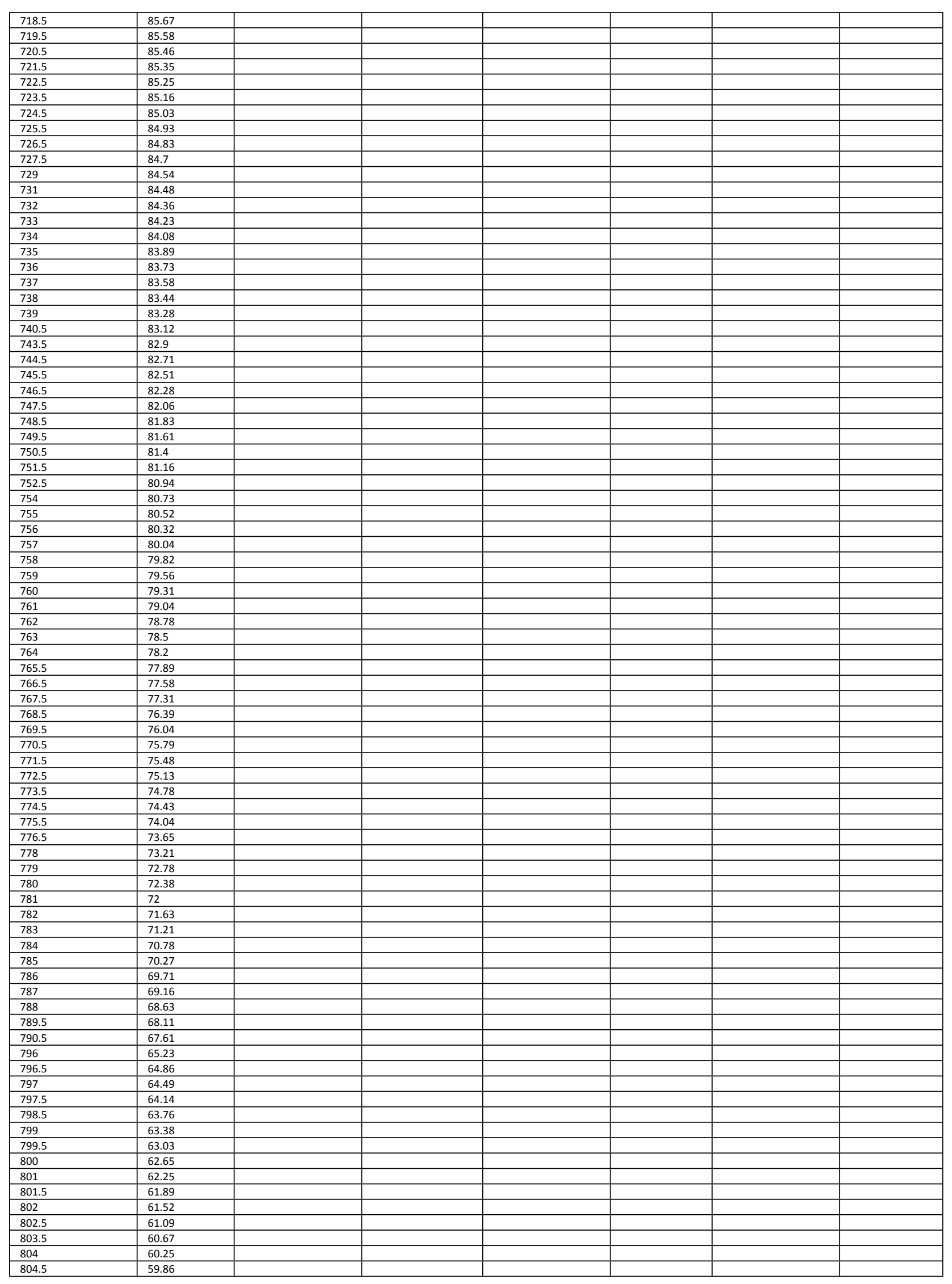




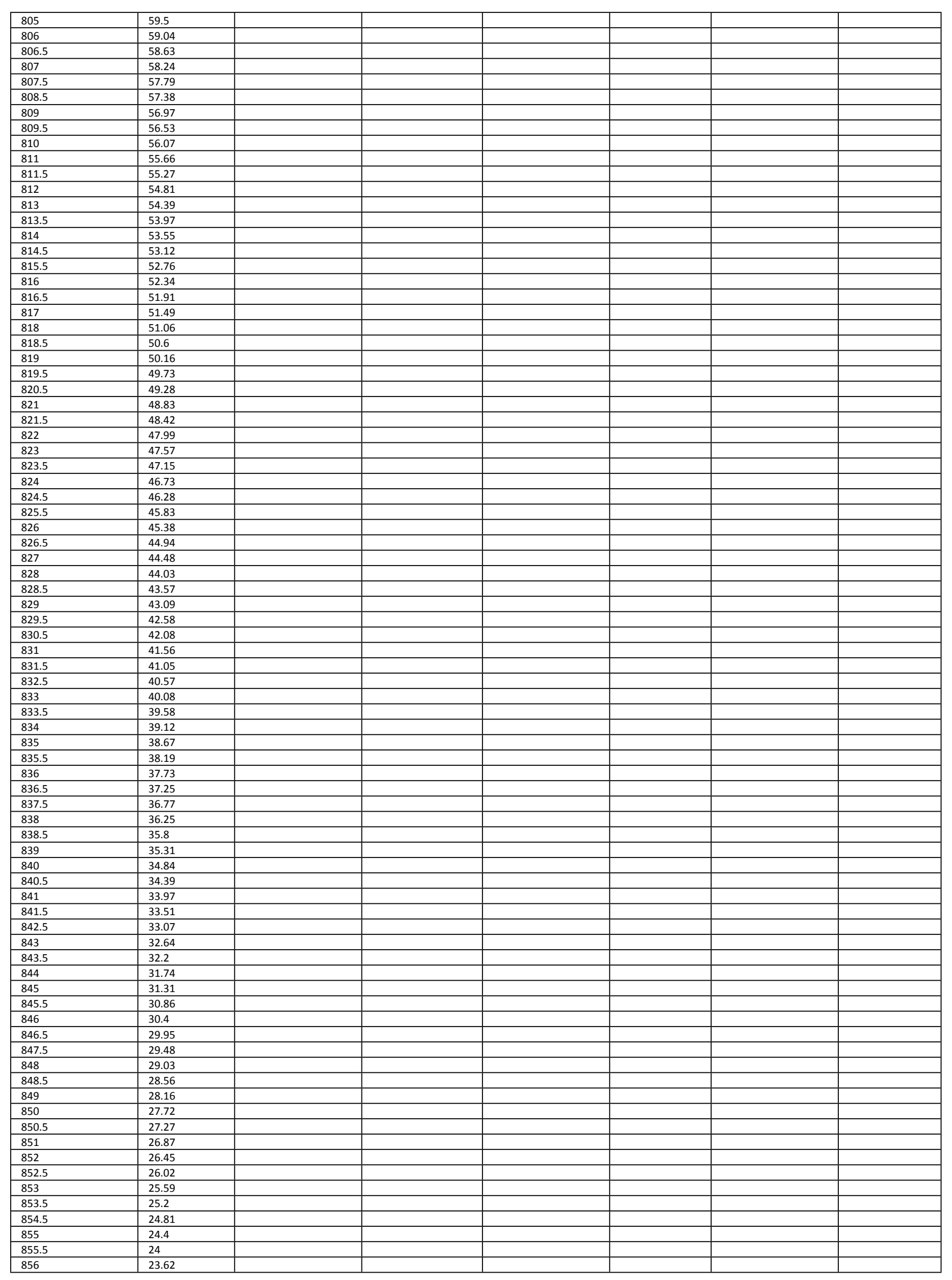




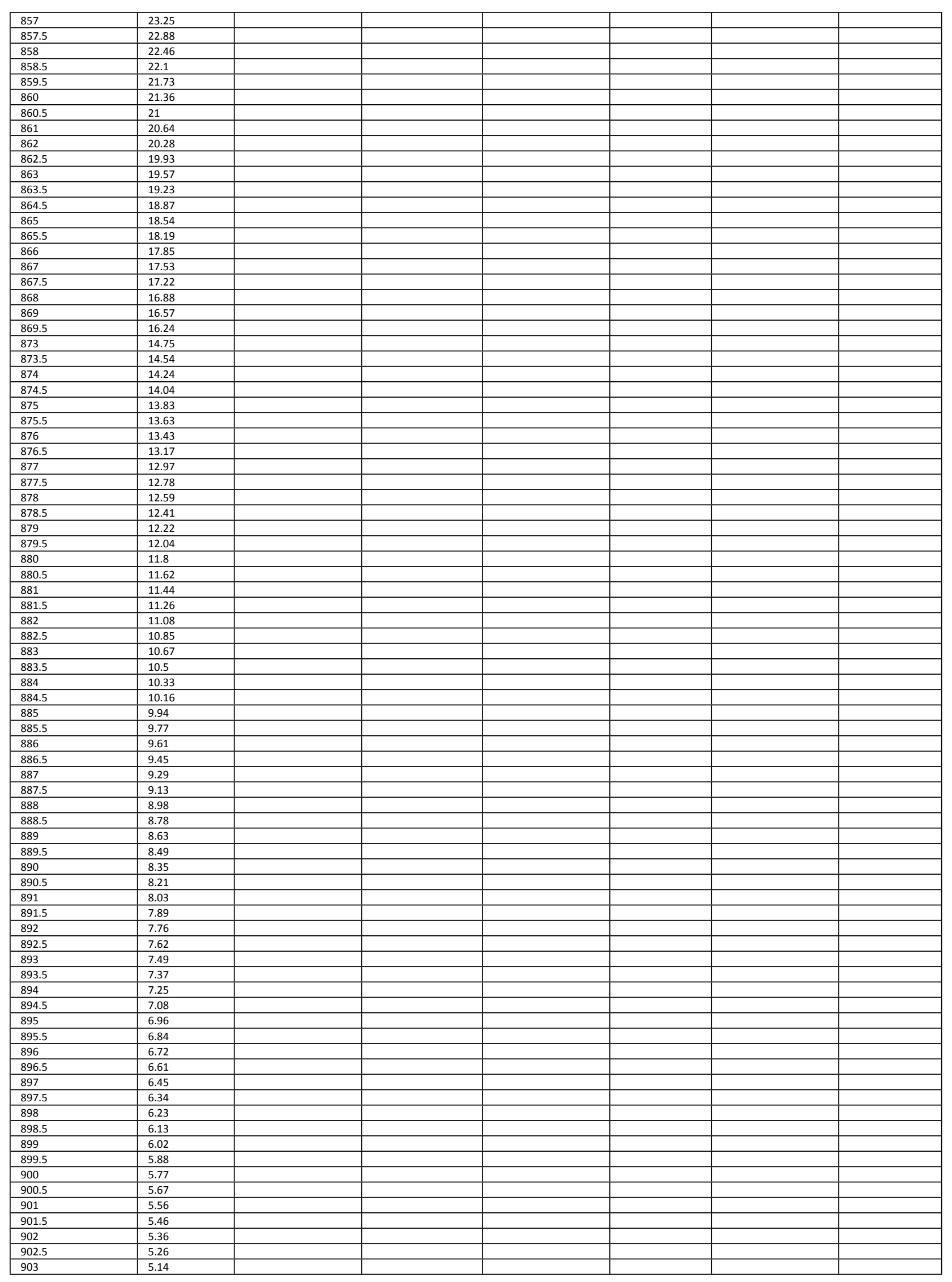




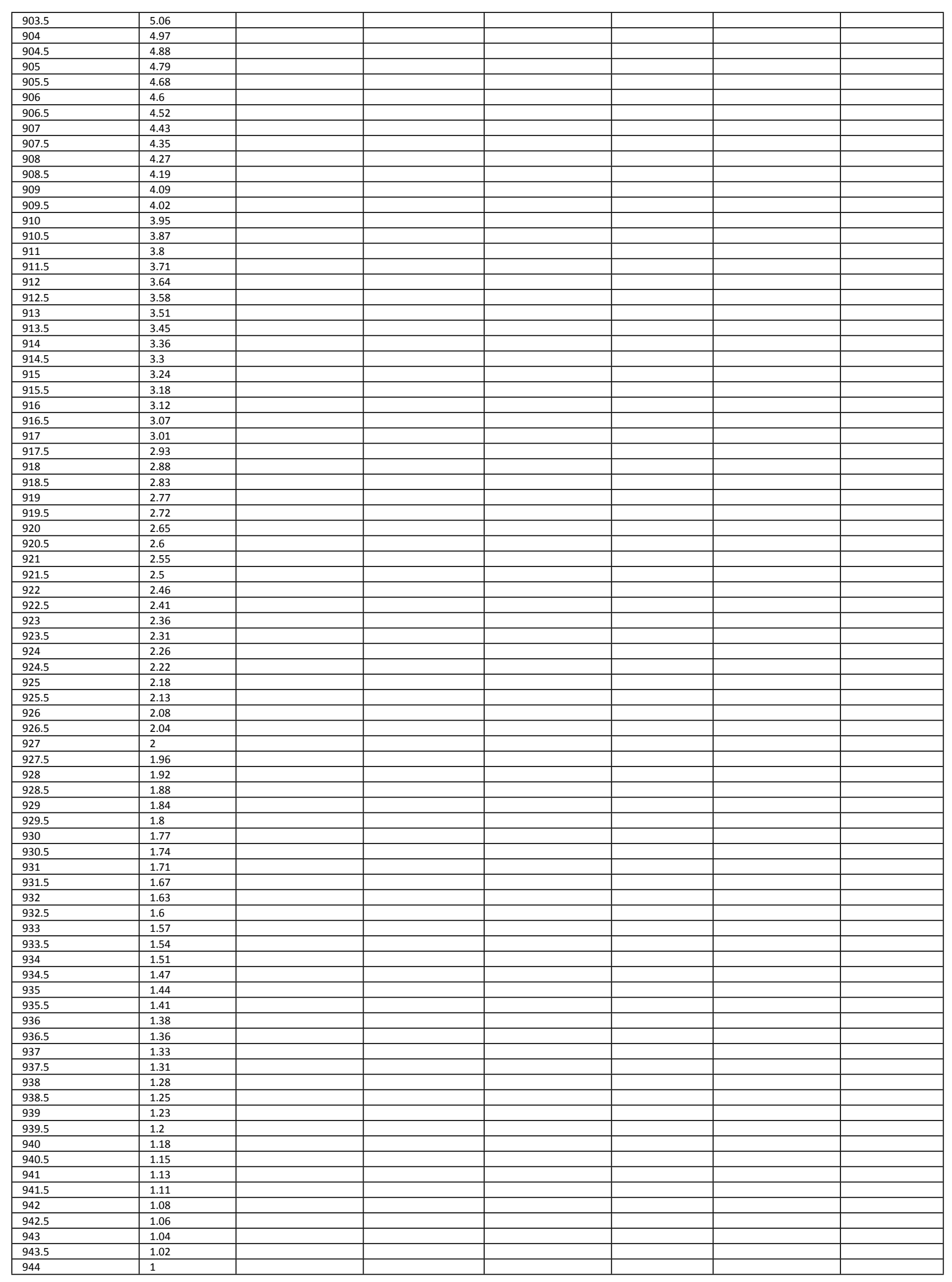




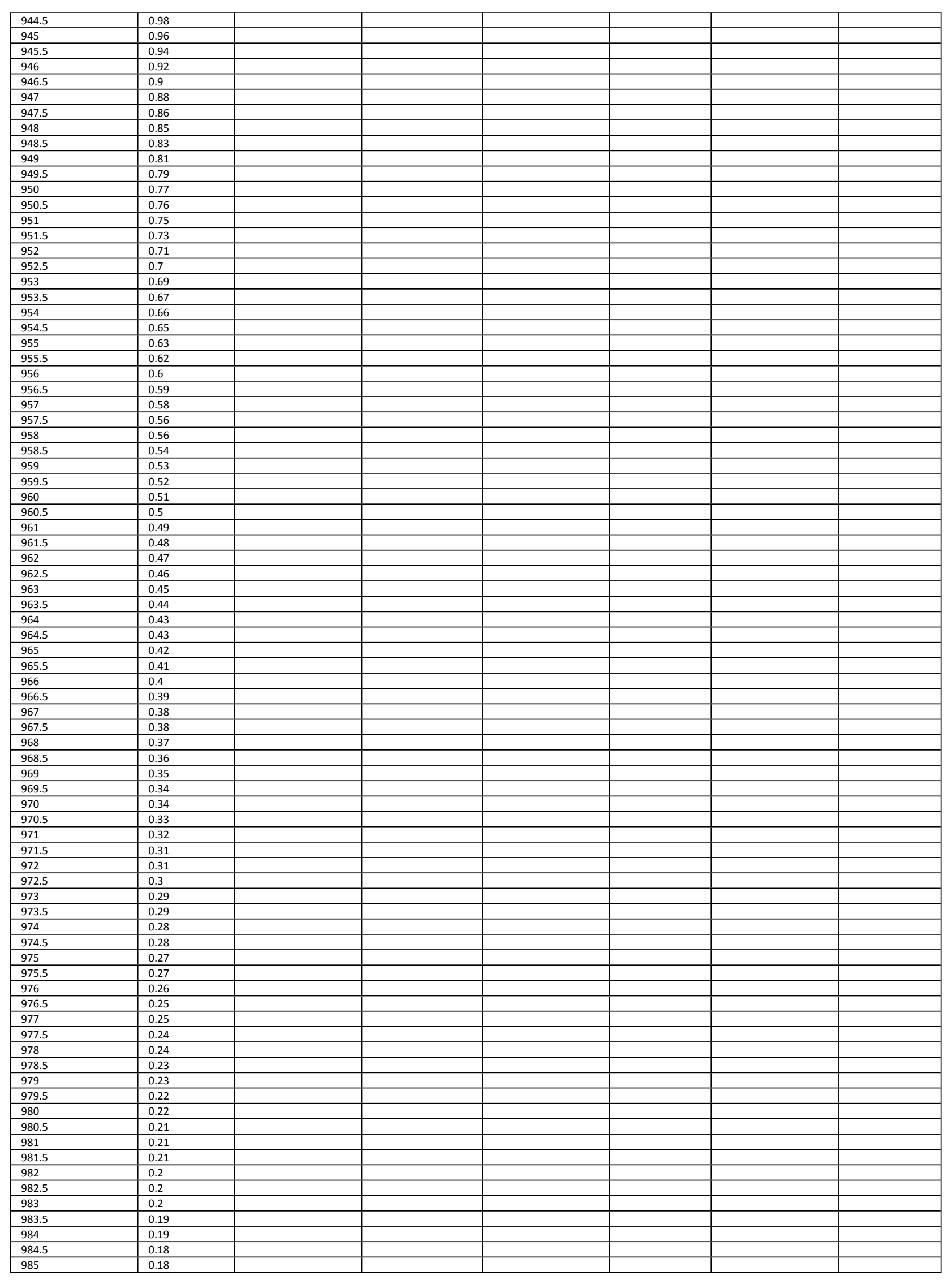




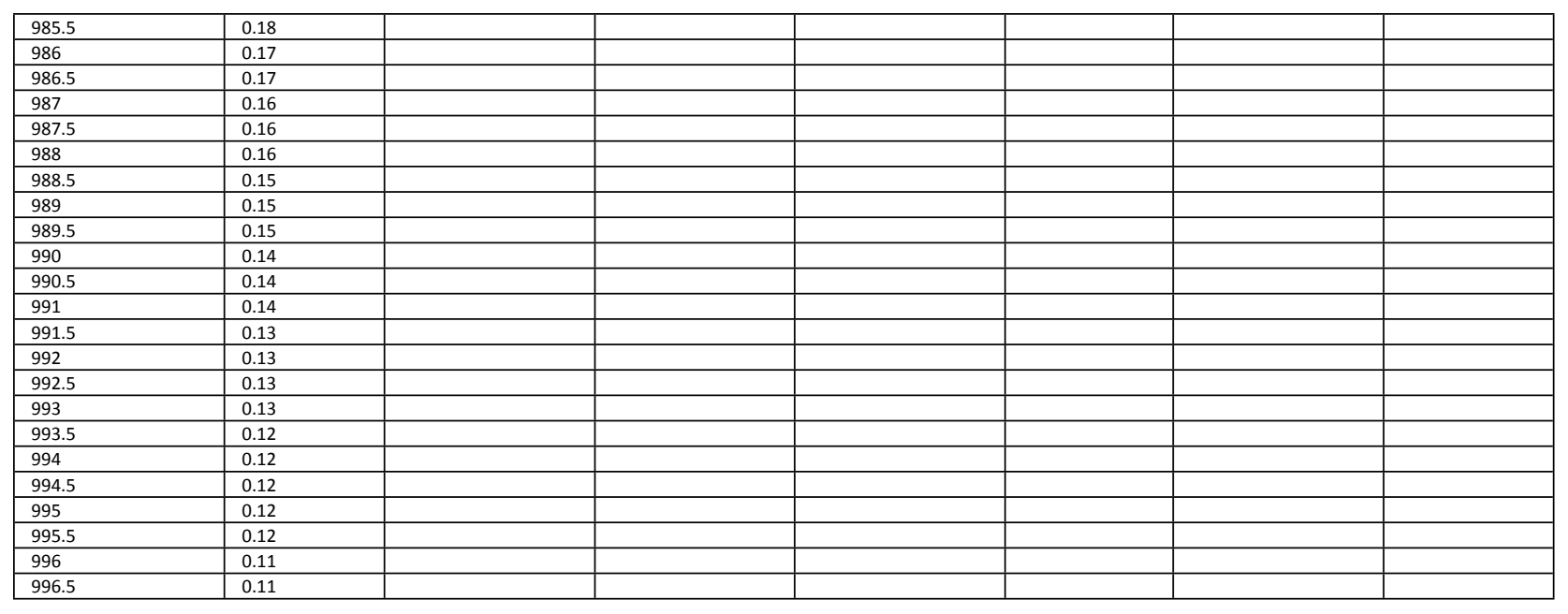

Table S6: EQE data for kesterite-inspired chalcogenides. Here, data for $\mathrm{Cu}_{2} \mathrm{BaSn}(\mathrm{S}, \mathrm{Se})_{4}$ were provided by the corresponding author(s) of ref ${ }^{16}$, data for $\mathrm{Cu}_{2}(\mathrm{Zn}, \mathrm{Cd}) \mathrm{SnS}_{4}$ were obtained by tracing Figure $6 \mathrm{~b}$ in ref ${ }^{17}$, data for $\mathrm{Cu}_{2} \mathrm{CdSnS}_{4}$ were provided by the corresponding author(s) of ref ${ }^{18}$, data for $\mathrm{Ag}_{2} \mathrm{ZnSnSe}_{4}$ were obtained by tracing Figure $3 \mathrm{c}$ in $\mathrm{ref}^{19}$, and data for $\mathrm{Cu}_{2} \mathrm{ZnGeSe}_{4}$ were provided by the corresponding author(s) of ref ${ }^{20}$.

\begin{tabular}{|c|c|c|c|c|c|c|c|c|c|}
\hline \multicolumn{2}{|c|}{$\mathrm{Cu}_{2} \mathrm{BaSn}(\mathrm{S}, \mathrm{Se})_{4}$} & \multicolumn{2}{|c|}{$\mathrm{Cu}_{2}(\mathrm{Zn}, \mathrm{Cd}) \mathrm{SnS}_{4}$} & \multicolumn{2}{|l|}{$\mathrm{Cu}_{2} \mathrm{CdSnS}_{4}$} & \multicolumn{2}{|l|}{$\mathrm{Ag}_{2} \mathrm{ZnSnSe}_{4}$} & \multicolumn{2}{|c|}{$\mathrm{Cu}_{2} \mathrm{ZnGeSe}_{4}$} \\
\hline $\begin{array}{l}\text { Wavelength } \\
(\mathrm{nm})\end{array}$ & $\mathrm{EQE}(\%)$ & $\begin{array}{l}\text { Wavelength } \\
(\mathrm{nm})\end{array}$ & EQE(\%) & $\begin{array}{l}\text { Wavelength } \\
(\mathrm{nm})\end{array}$ & $\mathrm{EQE}(\%)$ & $\begin{array}{l}\text { Wavelength } \\
(\mathrm{nm})\end{array}$ & EQE(\%) & $\begin{array}{l}\text { Wavelength } \\
(\mathrm{nm})\end{array}$ & $\mathrm{EQE}(\%)$ \\
\hline 300 & 5.36 & 301.21103 & 11.77407 & 300 & 1.90933 & 300.0093 & 12.5465 & 340 & 27.841 \\
\hline 310 & 7.97 & 306.35699 & 12.56688 & 305 & 3.9805 & 305.13491 & 14.73458 & 350 & 37.759 \\
\hline 320 & 10.56 & 309.93944 & 13.4032 & 310 & 3.42917 & 308.52436 & 16.45095 & 360 & 48.92 \\
\hline 330 & 13.36 & 314.70929 & 15.19374 & 315 & 2.8185 & 310.69825 & 17.13976 & 370 & 56.67 \\
\hline 340 & 15.9 & 318.11633 & 17.15623 & 320 & 2.40692 & 313.06251 & 18.65179 & 380 & 60.43 \\
\hline 350 & 18.26 & 320.45296 & 19.24147 & 325 & 4.28677 & 316.59319 & 20.14326 & 390 & 63.417 \\
\hline 360 & 20.66 & 322.97117 & 20.5202 & 330 & 3.64038 & 320.10711 & 21.9043 & 400 & 64.349 \\
\hline 370 & 25.64 & 325.16376 & 22.18681 & 335 & 6.08344 & 323.24455 & 23.33837 & 410 & 66.378 \\
\hline 380 & 34.12 & 326.29322 & 23.71064 & 340 & 7.81067 & 325.51457 & 24.34284 & 420 & 67.916 \\
\hline 390 & 40.4 & 327.29555 & 26.23402 & 345 & 9.05881 & 330.08897 & 26.31015 & 430 & 69.652 \\
\hline 400 & 43.49 & 328.09565 & 28.59233 & 350 & 10.96799 & 335.73969 & 28.71519 & 440 & 69.939 \\
\hline 410 & 45.23 & 329.06281 & 31.2965 & 355 & 13.05884 & 340.03171 & 30.75329 & 450 & 71.674 \\
\hline 420 & 47.13 & 330.09592 & 34.20049 & 360 & 15.55731 & 345.39673 & 32.59139 & 460 & 73.596 \\
\hline 430 & 49.18 & 333.47254 & 36.22649 & 365 & 18.11564 & 349.89242 & 34.46959 & 470 & 75.99 \\
\hline 440 & 51.2 & 336.69529 & 38.14791 & 370 & 20.54031 & 354.23729 & 35.89124 & 480 & 78.854 \\
\hline 450 & 53.4 & 339.30258 & 39.82536 & 375 & 22.56899 & 359.87949 & 37.42147 & 490 & 81.951 \\
\hline 460 & 55.73 & 341.85676 & 42.10854 & 380 & 25.23688 & 365.3753 & 39.06344 & 500 & 84.921 \\
\hline 470 & 58.15 & 345.02094 & 43.96461 & 385 & 27.78152 & 370.18034 & 40.5955 & 510 & 87.517 \\
\hline 480 & 60.59 & 348.65367 & 45.76833 & 390 & 30.36172 & 375.06053 & 41.41994 & 520 & 89.052 \\
\hline 490 & 63.48 & 352.86812 & 48.22565 & 395 & 32.93058 & 379.90315 & 42.14502 & 530 & 89.862 \\
\hline 500 & 67.21 & 356.56091 & 50.02937 & 400 & 34.54878 & 386.86524 & 42.60305 & 540 & 90.336 \\
\hline 510 & 71.33 & 360.55299 & 51.89843 & 405 & 36.89215 & 395.39952 & 42.91541 & 550 & 90.398 \\
\hline 520 & 75.18 & 364.03881 & 53.55836 & 410 & 38.5287 & 405.04322 & 43.01503 & 560 & 90.439 \\
\hline 530 & 78.03 & 368.56248 & 55.5058 & 415 & 40.04318 & 414.76998 & 43.23263 & 570 & 90.048 \\
\hline 540 & 80 & 375.3952 & 57.03337 & 420 & 41.74269 & 423.22119 & 43.61639 & 580 & 89.887 \\
\hline 550 & 80.61 & 383.66668 & 58.569 & 425 & 43.24789 & 432.20339 & 44.13897 & 590 & 89.531 \\
\hline 560 & 80.91 & 391.64241 & 59.90913 & 430 & 44.40126 & 440.83111 & 44.86236 & 600 & 89.426 \\
\hline 570 & 80.81 & 401.33598 & 61.48407 & 435 & 45.76965 & 449.6368 & 45.63444 & 610 & 89.251 \\
\hline 580 & 80.38 & 411.49116 & 62.95913 & 440 & 46.30657 & 458.66799 & 46.35268 & 620 & 89.024 \\
\hline 590 & 78.94 & 421.64633 & 64.13469 & 445 & 47.67453 & 466.58596 & 46.94864 & 630 & 88.957 \\
\hline 600 & 78.33 & 431.8015 & 65.10347 & 450 & 49.02897 & 475.25565 & 47.53865 & 640 & 88.175 \\
\hline 610 & 77.1 & 441.95667 & 66.25764 & 455 & 49.01474 & 483.05085 & 48.35347 & 650 & 88.096 \\
\hline 620 & 75.72 & 452.11184 & 67.56869 & 460 & 49.92468 & 493.66085 & 49.09512 & 660 & 87.459 \\
\hline 630 & 74.49 & 462.26702 & 68.97244 & 465 & 50.58605 & 502.90557 & 49.62236 & 670 & 87.328 \\
\hline 640 & 73.08 & 472.42219 & 70.3548 & 470 & 51.60611 & 511.83882 & 50.04534 & 680 & 86.78 \\
\hline 650 & 71.48 & 481.19256 & 72.16603 & 475 & 53.47854 & 521.30751 & 50.43807 & 690 & 85.922 \\
\hline 660 & 69.91 & 488.11654 & 73.81675 & 480 & 54.82428 & 530.0168 & 50.77296 & 700 & 85.062 \\
\hline 670 & 67.91 & 492.91271 & 75.29323 & 485 & 57.02677 & 539.22518 & 51.07251 & 710 & 84.386 \\
\hline 680 & 67.11 & 497.63938 & 76.9963 & 490 & 58.22916 & 548.19477 & 51.23478 & 720 & 83.299 \\
\hline 690 & 65.98 & 501.52822 & 78.81047 & 495 & 60.58834 & 557.62712 & 51.43505 & 730 & 81.784 \\
\hline 700 & 65.25 & 505.60712 & 80.60702 & 500 & 63.4986 & 566.37275 & 51.52383 & 740 & 80.149 \\
\hline
\end{tabular}




\begin{tabular}{|c|c|c|c|c|c|c|c|c|c|}
\hline 710 & 64.42 & 511.19648 & 82.51889 & 505 & 64.7411 & 575.54479 & 51.38973 & 750 & 79.747 \\
\hline 720 & 63.91 & 515.81247 & 84.32252 & 510 & 67.74601 & 585.05195 & 51.19608 & 760 & 76.893 \\
\hline 730 & 63.4 & 520.89005 & 86.17316 & 515 & 68.30426 & 593.94673 & 50.93656 & 770 & 76.715 \\
\hline 740 & 62.47 & 527.35244 & 87.9668 & 520 & 70.43027 & 602.22747 & 50.55192 & 780 & 73.917 \\
\hline 750 & 60.18 & 536.12281 & 89.44467 & 525 & 70.71871 & 610.89588 & 50.5287 & 790 & 72.754 \\
\hline 760 & 54.81 & 546.27798 & 90.31361 & 530 & 70.72961 & 620.90667 & 50.32775 & 800 & 70.827 \\
\hline 770 & 49.93 & 556.43316 & 90.66201 & 535 & 71.2538 & 629.78208 & 50.07553 & 810 & 68.764 \\
\hline 780 & 47.21 & 566.58833 & 90.7323 & 540 & 71.25208 & 639.08465 & 49.65994 & 820 & 65.702 \\
\hline 790 & 37.52 & 576.7435 & 90.74555 & 545 & 71.44733 & 650.12107 & 49.07855 & 830 & 62.968 \\
\hline 800 & 18.37 & 586.89867 & 90.62331 & 550 & 71.45404 & 657.26262 & 48.71636 & 840 & 58.061 \\
\hline 810 & 5.14 & 597.05384 & 90.55812 & 555 & 71.04005 & 667.07022 & 48.30816 & 850 & 52.347 \\
\hline 820 & 1.23 & 607.20902 & 90.42875 & 560 & 70.83446 & 675.4406 & 47.76282 & 860 & 44.051 \\
\hline 830 & 0.31 & 617.36419 & 89.99989 & 565 & 70.91575 & 685.95642 & 47.03927 & 870 & 35.059 \\
\hline 840 & 0 & 627.51936 & 89.7493 & 570 & 70.12934 & 694.55368 & 46.39765 & 880 & 25.738 \\
\hline 850 & 0 & 637.67453 & 89.44879 & 575 & 70.84654 & 701.93705 & 45.63444 & 890 & 18.261 \\
\hline 860 & 0 & 647.8297 & 89.06272 & 580 & 69.86521 & 709.58714 & 44.6855 & 900 & 11.575 \\
\hline 870 & 0 & 657.98487 & 88.49124 & 585 & 70.29923 & 719.91545 & 43.43922 & 910 & 6.7376 \\
\hline 880 & 0 & 668.14005 & 87.94829 & 590 & 69.88702 & 729.73031 & 42.14273 & 920 & 4.4911 \\
\hline 890 & 0 & 678.29522 & 87.33403 & 595 & 69.38786 & 743.37609 & 40.57709 & 930 & 2.4122 \\
\hline \multirow[t]{63}{*}{900} & 0 & 688.45039 & 86.59141 & 600 & 69.68023 & 760.0415 & 38.92019 & 940 & 1.4194 \\
\hline & & 698.60556 & 85.55643 & 605 & 68.90719 & 772.95561 & 37.68158 & 950 & 0.8828 \\
\hline & & 708.76073 & 84.6712 & 610 & 69.07797 & 779.90315 & 36.93353 & 960 & 0.58145 \\
\hline & & 718.91591 & 83.7931 & 615 & 68.37943 & 789.58479 & 36.11326 & 970 & 0.3677 \\
\hline & & 729.07108 & 82.92213 & 620 & 68.74729 & 804.65329 & 34.43627 & 980 & 0.27541 \\
\hline & & 739.22625 & 82.27222 & 625 & 68.30409 & 814.95656 & 33.07311 & 990 & 0.18132 \\
\hline & & 749.38142 & 81.38699 & 630 & 67.95392 & 820.38014 & 32.14325 & 1000 & 0.13902 \\
\hline & & 759.53659 & 80.40906 & 635 & 67.63483 & 830.35385 & 30.19706 & & \\
\hline & & 769.69177 & 79.53809 & 640 & 67.66826 & 834.6247 & 28.91239 & & \\
\hline & & 779.84694 & 78.57442 & 645 & 67.14404 & 838.94579 & 27.53494 & & \\
\hline & & 790.00211 & 77.56796 & 650 & 67.42732 & 843.49513 & 26.29286 & & \\
\hline & & 799.788 & 76.44044 & 655 & 67.00897 & 846.73123 & 25.15106 & & \\
\hline & & 805.34454 & 75.85802 & 660 & 66.60145 & 850.29913 & 24.064 & & \\
\hline & & 810.31245 & 75.17936 & 665 & 66.26727 & 854.47942 & 22.8852 & & \\
\hline & & 820.46762 & 73.63586 & 670 & 66.47363 & 859.29158 & 21.56355 & & \\
\hline & & 830.1612 & 71.95117 & 675 & 65.31366 & 869.36526 & 19.81677 & & \\
\hline & & 838.46997 & 70.1792 & 680 & 65.65595 & 874.8184 & 19.03323 & & \\
\hline & & 845.39396 & 68.43605 & 685 & 65.61256 & 879.66102 & 18.30816 & & \\
\hline & & 851.85634 & 66.62011 & 690 & 65.28074 & 885.64519 & 17.3681 & & \\
\hline & & 857.85712 & 64.89573 & 695 & 64.47615 & 892.82655 & 16.39576 & & \\
\hline & & 863.39631 & 63.09109 & 700 & 64.05141 & 900.35654 & 14.36766 & & \\
\hline & & 869.62789 & 61.39097 & 705 & 63.62277 & 903.87409 & 13.27795 & & \\
\hline & & 871.58496 & 59.98467 & 710 & 63.26364 & 908.9257 & 11.9834 & & \\
\hline & & 875.18792 & 59.09235 & 715 & 62.9468 & 914.55108 & 10.48709 & & \\
\hline & & 877.70587 & 57.72708 & 720 & 62.60655 & 919.47045 & 8.79871 & & \\
\hline & & 882.32185 & 55.90684 & 725 & 62.51602 & 925.1816 & 7.25076 & & \\
\hline & & 886.91253 & 53.93757 & 730 & 61.76752 & 930.06539 & 5.65086 & & \\
\hline & & 892.01543 & 51.74078 & 735 & 61.88351 & 936.55756 & 4.01039 & & \\
\hline & & 896.30614 & 49.90867 & 740 & 61.74361 & 943.08148 & 2.81374 & & \\
\hline & & 901.2474 & 47.99442 & 745 & 61.84797 & 949.81706 & 1.65449 & & \\
\hline & & 905.40179 & 46.08402 & 750 & 61.29306 & 959.70694 & 0.68126 & & \\
\hline & & 909.55618 & 44.24814 & 755 & 60.77771 & 970.03741 & 0.42838 & & \\
\hline & & 913.24897 & 42.65939 & 760 & 60.13732 & 987.87455 & 0.20752 & & \\
\hline & & 917.40336 & 40.8745 & 765 & 60.36084 & 1006.05253 & 0.18032 & & \\
\hline & & 921.55775 & 39.05039 & 770 & 60.24198 & 1024.2305 & 0.22696 & & \\
\hline & & 925.36222 & 37.82767 & 775 & 59.78129 & 1042.40848 & 0.22003 & & \\
\hline & & 929.03341 & 35.70947 & 780 & 59.679 & 1060.58645 & 0.20854 & & \\
\hline & & 932.63611 & 34.39199 & 785 & 59.22972 & 1078.76443 & 0.21851 & & \\
\hline & & 937.51665 & 32.36583 & 790 & 59.14752 & 1095.69267 & 0.16907 & & \\
\hline & & 940.16511 & 31.09464 & 795 & 58.52323 & & & & \\
\hline & & 942.79129 & 29.92978 & 800 & 58.26206 & & & & \\
\hline & & 946.48408 & 28.30181 & 805 & 58.20619 & & & & \\
\hline & & 950.63846 & 26.51692 & 810 & 57.75325 & & & & \\
\hline & & 955.25445 & 24.55549 & 815 & 57.33872 & & & & \\
\hline & & 959.40884 & 22.82943 & 820 & 56.8617 & & & & \\
\hline & & 963.56323 & 21.07199 & 825 & 56.11714 & & & & \\
\hline & & 968.17922 & 19.25175 & 830 & 55.36241 & & & & \\
\hline & & 972.7952 & 17.46289 & 835 & 53.89422 & & & & \\
\hline & & 977.41119 & 15.67403 & 840 & 52.30436 & & & & \\
\hline & & 982.02718 & 13.9793 & 845 & 50.05322 & & & & \\
\hline & & 987.56636 & 12.14776 & 850 & 47.99648 & & & & \\
\hline & & 994.02874 & 10.287 & 855 & 45.08037 & & & & \\
\hline & & 1000.49113 & 8.49347 & 860 & 41.99007 & & & & \\
\hline & & 1007.87671 & 6.8144 & 865 & 38.38253 & & & & \\
\hline & & 1017.10868 & 5.1322 & 870 & 34.37605 & & & & \\
\hline & & 1027.26385 & 3.74068 & 875 & 31.63707 & & & & \\
\hline & & 1037.41902 & 2.64153 & 880 & 28.12838 & & & & \\
\hline & & 1047.5742 & 1.88465 & 885 & 24.76512 & & & & \\
\hline & & 1057.72937 & 1.4344 & 890 & 21.48987 & & & & \\
\hline & & 1067.88454 & 1.08398 & 895 & 17.83318 & & & & \\
\hline & & 1078.03971 & 0.72786 & 900 & 15.36177 & & & & \\
\hline & & 1088.19488 & 0.69975 & 905 & 12.82745 & & & & \\
\hline & & 1097.42686 & 0.73811 & 910 & 10.69918 & & & & \\
\hline
\end{tabular}




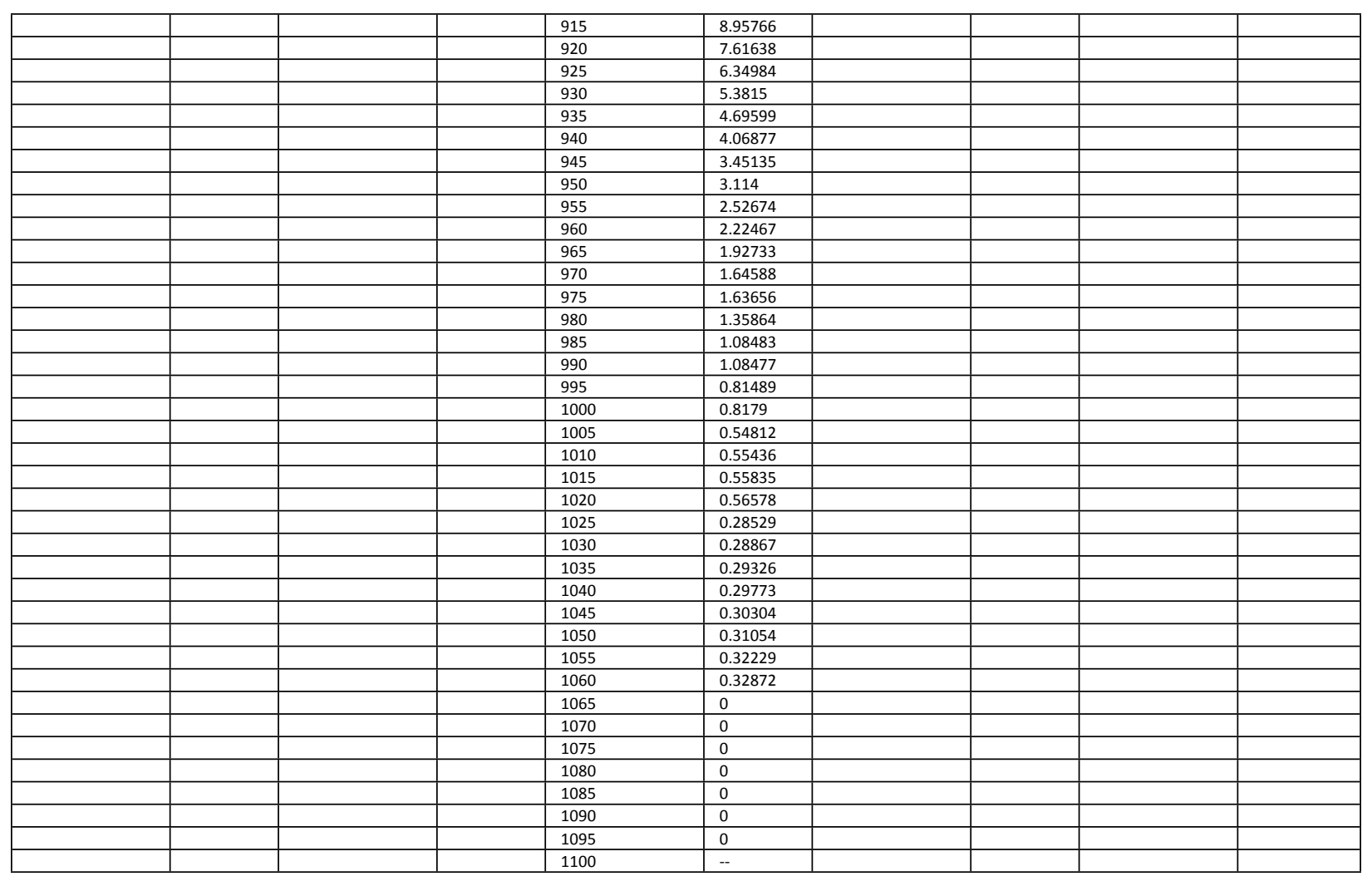

\section{2) IV data presented in Figure 1 (main manuscript)}

Table S7: IV data for binary quasi-1D chalcogenides. Here, data for $\mathrm{Sb}_{2} \mathrm{~S}_{3}$ were obtained by tracing Figure $5 b$ in ref ${ }^{1}$, data for $\mathrm{Sb}_{2}(\mathrm{~S}, \mathrm{Se})_{3}$ were obtained from the raw data available in the supporting information of $\mathrm{ref}^{2}$, and data for $\mathrm{Sb}_{2} \mathrm{Se}_{3}$ were provided by the corresponding author(s) of ref ${ }^{3}$.

\begin{tabular}{|c|c|c|c|c|c|}
\hline \multicolumn{2}{|l|}{$\mathrm{Sb}_{2} \mathrm{~S}_{3}$} & \multicolumn{2}{|c|}{$\mathrm{Sb}_{2}(\mathrm{~S}, \mathrm{Se})_{3}$} & \multicolumn{2}{|c|}{$\mathrm{Sb}_{2} \mathrm{Se}_{3}$} \\
\hline $\mathrm{V}(\mathrm{V})$ & $\mathrm{J}\left(\mathrm{mA} \mathrm{cm} \mathrm{cm}^{-2}\right)$ & $\mathrm{V}(\mathrm{V})$ & $\mathrm{J}\left(\mathrm{mA} \mathrm{cm} \mathrm{cm}^{-2}\right)$ & $\mathrm{V}(\mathrm{V})$ & $\mathrm{J}\left(\mathrm{mA} \mathrm{cm}{ }^{-2}\right)$ \\
\hline-0.0126 & 16.19562 & -0.1 & 23.96667 & -0.1 & 33.20784 \\
\hline 0.00849 & 16.16312 & -0.09 & 23.94444 & -0.09 & 33.18095 \\
\hline 0.02887 & 16.17118 & -0.08 & 23.92222 & -0.08 & 33.14253 \\
\hline 0.05063 & 16.1463 & -0.07 & 23.93333 & -0.07 & 33.08106 \\
\hline 0.07239 & 16.13756 & -0.06 & 23.88889 & $\begin{array}{l}-0.06 \\
\end{array}$ & 33.03112 \\
\hline 0.09416 & 16.13757 & -0.05 & 23.87778 & -0.05 & 32.95428 \\
\hline 0.11592 & 16.13186 & -0.04 & 23.86667 & -0.04 & 32.91971 \\
\hline 0.13768 & 16.13116 & -0.03 & 23.82222 & -0.03 & 32.81598 \\
\hline 0.15945 & 16.11543 & -0.02 & 23.82222 & -0.02 & 32.71994 \\
\hline 0.18121 & 16.09253 & -0.01 & 23.8 & -0.01 & 32.62005 \\
\hline 0.20297 & 16.05173 & $-5.308 \mathrm{E}-16$ & 23.77778 & $-1.04 \mathrm{E}-17$ & 32.57395 \\
\hline 0.22474 & 16.0496 & 0.01 & 23.74444 & 0.01 & 32.51633 \\
\hline 0.2465 & 16.01381 & 0.02 & 23.73333 & 0.02 & 32.42797 \\
\hline 0.26827 & 15.96513 & 0.03 & 23.72222 & 0.03 & 32.33192 \\
\hline 0.29003 & 15.94438 & 0.04 & 23.71111 & 0.04 & 32.22051 \\
\hline 0.31179 & 15.89284 & 0.05 & 23.66667 & 0.05 & 32.12831 \\
\hline 0.33356 & 15.81982 & 0.06 & 23.64444 & 0.06 & 32.06685 \\
\hline 0.35532 & 15.7732 & 0.07 & 23.63333 & 0.07 & 32.02075 \\
\hline 0.37709 & 15.73805 & 0.08 & 23.61111 & 0.08 & 31.93623 \\
\hline 0.39885 & 15.65643 & 0.09 & 23.58889 & 0.09 & 31.84403 \\
\hline 0.42062 & 15.5075 & 0.1 & 23.57778 & 0.1 & 31.77488 \\
\hline 0.44176 & 15.30877 & 0.11 & 23.55556 & 0.11 & 31.67883 \\
\hline 0.46208 & 15.09168 & 0.12 & 23.52222 & 0.12 & 31.602 \\
\hline 0.48164 & 14.83063 & 0.13 & 23.5 & 0.13 & 31.46754 \\
\hline 0.49992 & 14.57893 & 0.14 & 23.45556 & 0.14 & 31.37918 \\
\hline 0.5172 & 14.17804 & 0.15 & 23.43333 & 0.15 & 31.32155 \\
\hline 0.53324 & 13.67812 & 0.16 & 23.41111 & 0.16 & 31.21014 \\
\hline 0.55291 & 12.93203 & 0.17 & 23.37778 & 0.17 & 31.10257 \\
\hline 0.57073 & 12.25858 & 0.18 & 23.34444 & 0.18 & 31.00653 \\
\hline
\end{tabular}




\begin{tabular}{|c|c|c|c|c|c|}
\hline 0.5856 & 11.47748 & 0.19 & 23.31111 & 0.19 & 30.92201 \\
\hline 0.6014 & 10.58983 & 0.2 & 23.28889 & 0.2 & 30.87207 \\
\hline 0.61399 & 9.73662 & 0.21 & 23.26667 & 0.21 & 30.7184 \\
\hline 0.62614 & 8.79059 & 0.22 & 23.2 & 0.22 & 30.59931 \\
\hline 0.63738 & 7.82377 & 0.23 & 23.18889 & 0.23 & 30.45332 \\
\hline 0.64908 & 6.782 & 0.24 & 23.15556 & 0.24 & 30.35344 \\
\hline 0.66111 & 5.64616 & 0.25 & 23.11111 & 0.25 & 30.10757 \\
\hline 0.67244 & 4.46583 & 0.26 & 23.07778 & 0.26 & 29.88475 \\
\hline 0.68319 & 3.069 & 0.27 & 23.02222 & 0.27 & 29.71571 \\
\hline 0.6901 & 2.52547 & 0.28 & 22.97778 & 0.28 & 29.5121 \\
\hline 0.69641 & 1.64085 & 0.29 & 22.93333 & 0.29 & 29.24318 \\
\hline 0.70239 & 1.05304 & 0.3 & 22.88889 & 0.3 & 28.89358 \\
\hline 0.70775 & 0.07763 & 0.31 & 22.83333 & 0.31 & 28.50173 \\
\hline 0.70903 & -0.20519 & 0.32 & 22.77778 & 0.32 & 28.09066 \\
\hline \multirow[t]{43}{*}{0.71089} & -0.61988 & 0.33 & 22.72222 & 0.33 & 27.57972 \\
\hline & & 0.34 & 22.65556 & 0.34 & 26.9151 \\
\hline & & 0.35 & 22.6 & 0.35 & 25.93546 \\
\hline & & 0.36 & 22.53333 & 0.36 & 24.85978 \\
\hline & & 0.37 & 22.45556 & 0.37 & 23.27699 \\
\hline & & 0.38 & 22.37778 & 0.38 & 20.89512 \\
\hline & & 0.39 & 22.27778 & 0.39 & 16.67691 \\
\hline & & 0.4 & 22.17778 & 0.4 & 1.36573 \\
\hline & & 0.41 & 22.06667 & 0.41 & -38.41721 \\
\hline & & 0.42 & 21.92222 & & \\
\hline & & 0.43 & 21.75556 & & \\
\hline & & 0.44 & 21.58889 & & \\
\hline & & 0.45 & 21.36667 & & \\
\hline & & 0.46 & 21.15556 & & \\
\hline & & 0.47 & 20.87778 & & \\
\hline & & 0.48 & 20.52222 & & \\
\hline & & 0.49 & 20.18889 & & \\
\hline & & 0.5 & 19.75556 & & \\
\hline & & 0.51 & 19.24444 & & \\
\hline & & 0.52 & 18.65556 & & \\
\hline & & 0.53 & 17.98889 & & \\
\hline & & 0.54 & 17.17778 & & \\
\hline & & 0.55 & 16.28889 & & \\
\hline & & 0.56 & 15.32222 & & \\
\hline & & 0.57 & 14.2 & & \\
\hline & & 0.58 & 12.94444 & & \\
\hline & & 0.59 & 11.58889 & & \\
\hline & & 0.6 & 10.09667 & & \\
\hline & & 0.61 & 8.54444 & & \\
\hline & & 0.62 & 6.77 & & \\
\hline & & 0.63 & 4.99111 & & \\
\hline & & 0.64 & 3.06889 & & \\
\hline & & 0.65 & 1.065 & & \\
\hline & & 0.66 & -1.04433 & & \\
\hline & & 0.67 & -3.23444 & & \\
\hline & & 0.68 & -5.50333 & & \\
\hline & & 0.69 & -7.86222 & & \\
\hline & & 0.7 & -10.37667 & & \\
\hline & & 0.71 & -12.73333 & & \\
\hline & & 0.72 & -15.13333 & & \\
\hline & & 0.73 & -17.48889 & & \\
\hline & & 0.74 & -19.8 & & \\
\hline & & 0.75 & -21.55556 & & \\
\hline
\end{tabular}

Table S7: IV data for binary quasi-2D chalcogenides. Here, data for SnS were obtained by tracing Figure $3 a$ in ref $^{4}$ and data for GeSe were provided by the corresponding author(s) of ref ${ }^{5}$.

\begin{tabular}{|l|l|l|l|}
\hline \multicolumn{2}{|l|}{$\mathrm{SnS}$} & $\mathrm{GeSe}$ & $\mathrm{J}\left(\mathrm{mA} \mathrm{cm}^{-2}\right)$ \\
\hline $\mathrm{V}(\mathrm{V})$ & $\mathrm{J}\left(\mathrm{mA} \mathrm{cm}{ }^{-2}\right)$ & $\mathrm{V}(\mathrm{V})$ & 25.29033 \\
\hline-0.2 & 25.28 & -0.10047 & 25.2173 \\
\hline-0.19295 & 25.27 & -0.09434 & 25.17735 \\
\hline-0.18595 & 25.26 & -0.08816 & 25.14427 \\
\hline-0.1789 & 25.26 & -0.08203 & 25.10305 \\
\hline-0.17185 & 25.21 & -0.07585 & 25.06539 \\
\hline-0.1648 & 25.21 & -0.06973 & 25.02443 \\
\hline-0.1578 & 25.2 & -0.0636 & 24.98677 \\
\hline-0.15075 & 25.18 & -0.05742 & 24.94936 \\
\hline-0.1437 & 25.16 & -0.05129 & -0.04511 \\
\hline-0.1367 & 25.14 & -0.03898 & 24.90636 \\
\hline-0.12965 & 25.12 & & 24.87252 \\
\hline
\end{tabular}




\begin{tabular}{|c|c|c|c|}
\hline-0.1226 & 25.1 & -0.03285 & 24.83155 \\
\hline-0.1156 & 25.1 & -0.02667 & 24.79288 \\
\hline-0.10855 & 25.06 & -0.02055 & 24.75089 \\
\hline-0.1015 & 25.06 & -0.01437 & 24.70102 \\
\hline-0.09445 & 25.03 & -0.00824 & 24.66183 \\
\hline-0.08745 & 25.01 & -0.00211 & 24.62163 \\
\hline-0.0804 & 24.98 & 0.00407 & 24.57659 \\
\hline-0.07335 & 24.95 & 0.0102 & 24.53257 \\
\hline-0.06635 & 24.94 & 0.01638 & 24.48244 \\
\hline-0.0593 & 24.91 & 0.02251 & 24.44275 \\
\hline-0.05225 & 24.9 & 0.02863 & 24.39059 \\
\hline-0.04525 & 24.87 & 0.03481 & 24.33919 \\
\hline-0.0382 & 24.86 & 0.04094 & 24.28906 \\
\hline-0.03115 & 24.83 & 0.04712 & 24.23919 \\
\hline-0.0241 & 24.8 & 0.05325 & 24.18524 \\
\hline-0.0171 & 24.77 & 0.05938 & 24.13232 \\
\hline-0.01005 & 24.74 & 0.06556 & 24.07735 \\
\hline-0.003 & 24.71 & 0.07169 & 24.01501 \\
\hline 0.004 & 24.69 & 0.07786 & 23.96234 \\
\hline 0.01105 & 24.65 & 0.08399 & 23.90356 \\
\hline 0.0181 & 24.64 & 0.09012 & 23.84148 \\
\hline 0.02515 & 24.57 & 0.0963 & 23.7827 \\
\hline 0.03215 & 24.56 & 0.10243 & 23.71298 \\
\hline 0.0392 & 24.53 & 0.10861 & 23.64198 \\
\hline 0.04625 & 24.5 & 0.11474 & 23.57354 \\
\hline 0.05325 & 24.44 & 0.12087 & 23.50102 \\
\hline 0.0603 & 24.39 & 0.12704 & 23.42341 \\
\hline 0.06735 & 24.36 & 0.13317 & 23.3486 \\
\hline 0.07435 & 24.32 & 0.13935 & 23.26845 \\
\hline 0.0814 & 24.26 & 0.14548 & 23.1799 \\
\hline 0.08845 & 24.24 & $\begin{array}{l}0.15161 \\
\end{array}$ & 23.09313 \\
\hline 0.0955 & 24.16 & 0.15779 & 23.00153 \\
\hline 0.1025 & 24.14 & 0.16392 & 22.90153 \\
\hline 0.10955 & 24.07 & 0.1701 & 22.79949 \\
\hline 0.1166 & 24 & 0.17622 & 22.6888 \\
\hline 0.1236 & 23.93 & 0.18235 & 22.57048 \\
\hline 0.13065 & 23.84 & 0.18853 & 22.44962 \\
\hline 0.1377 & 23.74 & 0.19466 & 22.31501 \\
\hline 0.1447 & 23.64 & 0.20084 & 22.16718 \\
\hline 0.15175 & 23.51 & 0.20697 & 22.00636 \\
\hline 0.1588 & 23.38 & 0.2131 & 21.84555 \\
\hline 0.16585 & 23.24 & 0.21928 & 21.66031 \\
\hline 0.17285 & 23.09 & 0.2254 & 21.45802 \\
\hline 0.1799 & 22.89 & 0.23158 & 21.23206 \\
\hline 0.18695 & 22.69 & 0.23771 & 20.9944 \\
\hline 0.19395 & 22.44 & 0.24384 & 20.72774 \\
\hline 0.201 & 22.14 & 0.25002 & 20.42977 \\
\hline 0.20805 & 21.84 & 0.25615 & 20.10356 \\
\hline 0.2151 & 21.46 & 0.26233 & 19.73791 \\
\hline 0.2221 & 21.06 & 0.26846 & 19.33588 \\
\hline 0.22915 & 20.55 & 0.27458 & 18.88779 \\
\hline 0.2362 & 19.97 & 0.28076 & 18.3916 \\
\hline 0.2432 & 19.35 & 0.28689 & 17.84427 \\
\hline 0.25025 & 18.59 & 0.29307 & 17.22697 \\
\hline 0.2573 & 17.76 & 0.2992 & 16.55165 \\
\hline 0.2643 & 16.79 & 0.30533 & 15.80178 \\
\hline 0.27135 & 15.69 & 0.31151 & 14.96794 \\
\hline 0.2784 & 14.46 & 0.31764 & 14.05394 \\
\hline 0.28545 & 13.04 & 0.32381 & 13.03919 \\
\hline 0.29245 & 11.5 & 0.32994 & 11.93053 \\
\hline 0.2995 & 9.71 & 0.33607 & 10.71628 \\
\hline 0.30655 & 7.75 & 0.34225 & 9.38906 \\
\hline 0.31355 & 5.59 & 0.34838 & 7.94733 \\
\hline 0.3206 & 3.18 & 0.35456 & 6.38448 \\
\hline 0.32765 & 0.56771 & 0.36069 & 4.71603 \\
\hline 0.33465 & -2.29 & 0.36682 & 2.92875 \\
\hline 0.3417 & -5.42 & 0.37299 & 0.97761 \\
\hline 0.34875 & -8.8 & 0.37912 & -1.06997 \\
\hline 0.3558 & -12.38 & 0.3853 & -3.24809 \\
\hline 0.3628 & -16.25 & 0.39143 & -5.50305 \\
\hline 0.36985 & -20.38 & 0.39756 & -7.88804 \\
\hline 0.3769 & -24.75 & 0.40374 & -10.42061 \\
\hline 0.3839 & -29.34 & 0.40987 & -13.06056 \\
\hline \multirow[t]{9}{*}{0.39095} & -34.25 & 0.41605 & -15.79847 \\
\hline & & 0.42217 & -18.63003 \\
\hline & & 0.4283 & -21.53079 \\
\hline & & 0.43448 & -24.53359 \\
\hline & & 0.44061 & -27.60102 \\
\hline & & 0.44679 & -30.75191 \\
\hline & & 0.45292 & -33.93206 \\
\hline & & 0.45905 & -37.14631 \\
\hline & & 0.46523 & -40.43333 \\
\hline
\end{tabular}




\begin{tabular}{|l|l|l|l|}
\hline & & 0.47136 & -43.70941 \\
\hline & & 0.47753 & -47.0514 \\
\hline & & 0.48366 & -50.40178 \\
\hline & & 0.48979 & -53.74987 \\
\hline & & 0.49597 & -57.1659 \\
\hline & 0.5021 & -60.58677 & \\
\hline
\end{tabular}

Table S9: IV data for ternary quasi-2D chalcogenides. Here, data for $\mathrm{CuSbS}_{2}$ were obtained by tracing Figure $15 a$ in ref ${ }^{6}$ and data for $\mathrm{CuSbSe}_{2}$ were obtained by tracing Figure $5 \mathrm{~b}$ in ref ${ }^{7}$.

\begin{tabular}{|c|c|c|c|}
\hline \multicolumn{2}{|c|}{$\mathrm{CuSbS}_{2}$} & \multicolumn{2}{|c|}{$\mathrm{CuSbSe}_{2}$} \\
\hline $\mathrm{V}(\mathrm{V})$ & $\mathrm{J}\left(\mathrm{mA} \mathrm{cm} \mathrm{cm}^{-2}\right)$ & $\mathrm{V}(\mathrm{V})$ & $\mathrm{J}\left(\mathrm{mA} \mathrm{cm}{ }^{-2}\right)$ \\
\hline-0.10204 & 15.9662 & -0.19766 & 28.01568 \\
\hline-0.09096 & 15.88896 & -0.19051 & 27.89007 \\
\hline-0.07989 & 15.88363 & -0.18344 & 27.88081 \\
\hline-0.06881 & 15.82503 & -0.17637 & 27.88081 \\
\hline-0.05773 & 15.77709 & -0.1693 & 27.8623 \\
\hline-0.04665 & 15.7105 & -0.16223 & 27.74197 \\
\hline-0.03557 & 15.68386 & -0.15516 & 27.65867 \\
\hline-0.0245 & 15.71582 & -0.14809 & 27.65867 \\
\hline-0.01342 & 15.60928 & -0.14102 & 27.6309 \\
\hline-0.00213 & 15.59989 & -0.13395 & 27.4828 \\
\hline 0.00874 & 15.46544 & -0.12688 & 27.37173 \\
\hline 0.01981 & 15.44147 & -0.11981 & 27.32545 \\
\hline 0.03089 & 15.43614 & -0.11273 & 27.32545 \\
\hline 0.04197 & 15.3296 & -0.10566 & 27.32545 \\
\hline 0.05305 & 15.26833 & -0.09859 & 27.31619 \\
\hline 0.06413 & 15.2417 & -0.09152 & 27.15884 \\
\hline 0.0752 & 15.16179 & -0.08445 & 27.04776 \\
\hline 0.08628 & 15.03393 & -0.07738 & 27.00148 \\
\hline 0.09736 & 14.98865 & -0.07031 & 26.99223 \\
\hline 0.10844 & 14.90075 & -0.06324 & 27.02 \\
\hline 0.11951 & 14.80752 & -0.05617 & 27.00148 \\
\hline 0.13059 & 14.67967 & -0.0491 & 26.85339 \\
\hline 0.14167 & 14.61841 & -0.04203 & 26.77008 \\
\hline 0.15275 & 14.47191 & -0.03496 & 26.77008 \\
\hline 0.16382 & 14.31475 & -0.02789 & 26.71455 \\
\hline 0.1749 & 14.18157 & -0.02082 & 26.53868 \\
\hline 0.18598 & 14.04573 & -0.01375 & 26.43686 \\
\hline 0.19706 & 13.87525 & -0.00668 & 26.46463 \\
\hline 0.20814 & 13.70744 & $8.6 \mathrm{E}-4$ & 26.37577 \\
\hline 0.21921 & 13.49435 & 0.00746 & 26.3443 \\
\hline 0.23029 & 13.28659 & 0.01453 & 26.16844 \\
\hline 0.24137 & 13.04686 & 0.0216 & 26.1129 \\
\hline 0.25245 & 12.79115 & 0.02867 & 25.98332 \\
\hline 0.26352 & 12.52479 & 0.03574 & 25.8815 \\
\hline 0.2746 & 12.25843 & 0.04281 & 25.8815 \\
\hline 0.28568 & 11.92015 & 0.04988 & 25.8815 \\
\hline 0.29676 & 11.59252 & 0.05695 & 25.76117 \\
\hline 0.30784 & 11.21428 & 0.06402 & 25.62233 \\
\hline 0.31891 & 10.83605 & 0.07109 & 25.60382 \\
\hline 0.32999 & 10.43384 & 0.07816 & 25.42795 \\
\hline 0.34107 & 10.00766 & 0.08523 & 25.2706 \\
\hline 0.35215 & 9.5282 & 0.0923 & 25.2706 \\
\hline 0.36322 & 9.0381 & 0.09937 & 25.1225 \\
\hline 0.3743 & 8.51336 & 0.10644 & 24.93738 \\
\hline 0.38538 & 7.93535 & 0.11352 & 24.91887 \\
\hline 0.39646 & 7.34935 & 0.12059 & 24.78929 \\
\hline 0.40753 & 6.76868 & 0.12766 & 24.54863 \\
\hline 0.41861 & 6.1667 & 0.13473 & 24.4283 \\
\hline 0.42919 & 5.53489 & 0.1418 & 24.38202 \\
\hline 0.43926 & 4.90787 & 0.14887 & 24.25243 \\
\hline 0.44933 & 4.2955 & 0.15594 & 24.05806 \\
\hline 0.4589 & 3.69159 & 0.16301 & 23.84517 \\
\hline 0.46846 & 3.05904 & 0.17008 & 23.61377 \\
\hline 0.47853 & 2.38221 & 0.17715 & 23.33608 \\
\hline 0.4876 & 1.75006 & 0.18422 & 23.00286 \\
\hline 0.49565 & 1.12744 & 0.19129 & 22.7437 \\
\hline 0.5039 & 0.46281 & 0.19836 & 22.37345 \\
\hline 0.50916 & 0.0903 & 0.20551 & 22.04949 \\
\hline 0.51429 & -0.28177 & 0.2125 & 21.55892 \\
\hline 0.52385 & -1.01878 & 0.21999 & 21.11331 \\
\hline 0.53191 & -1.66338 & 0.22664 & 20.64257 \\
\hline 0.53996 & -2.33362 & 0.2343 & 20.14737 \\
\hline
\end{tabular}




\begin{tabular}{|c|c|c|c|}
\hline 0.54802 & -2.99653 & 0.24072 & 19.67377 \\
\hline 0.55557 & -3.6134 & 0.24694 & 19.00004 \\
\hline 0.56313 & -4.23812 & 0.25348 & 18.23263 \\
\hline 0.57118 & -4.91568 & 0.25963 & 17.4844 \\
\hline 0.57874 & -5.56394 & 0.26523 & 16.70411 \\
\hline 0.58579 & -6.18342 & 0.27024 & 16.1071 \\
\hline 0.59284 & -6.82384 & 0.27643 & 14.84364 \\
\hline 0.59988 & -7.46844 & 0.28229 & 14.16332 \\
\hline 0.60693 & -8.12559 & 0.28806 & 13.2678 \\
\hline 0.61398 & -8.79531 & 0.29204 & 12.43244 \\
\hline 0.62103 & -9.46083 & 0.29499 & 11.88633 \\
\hline 0.62808 & -10.14729 & 0.29852 & 11.11808 \\
\hline 0.63526 & -10.77201 & 0.30113 & 10.43445 \\
\hline 0.64185 & -11.44672 & 0.305 & 9.60317 \\
\hline 0.64903 & -12.14471 & 0.30786 & 8.72869 \\
\hline 0.6554 & -12.83033 & 0.31134 & 7.84606 \\
\hline \multirow[t]{40}{*}{0.6607} & -13.32843 & 0.31414 & 6.99913 \\
\hline & & 0.31723 & 6.06195 \\
\hline & & 0.32076 & 5.18031 \\
\hline & & 0.32356 & 4.34033 \\
\hline & & 0.3271 & 3.47951 \\
\hline & & 0.32896 & 2.64878 \\
\hline & & 0.33255 & 1.85507 \\
\hline & & 0.33505 & 1.01046 \\
\hline & & 0.33743 & 0.10028 \\
\hline & & 0.33917 & -0.49828 \\
\hline & & 0.34117 & -1.46051 \\
\hline & & 0.34448 & -2.65865 \\
\hline & & 0.34625 & -3.38617 \\
\hline & & 0.34828 & -4.20912 \\
\hline & & 0.3498 & -4.94119 \\
\hline & & 0.35122 & -5.64465 \\
\hline & & 0.35332 & -6.49621 \\
\hline & & 0.35476 & -7.24904 \\
\hline & & 0.35684 & -8.3104 \\
\hline & & 0.35984 & -9.5352 \\
\hline & & 0.36223 & -10.60416 \\
\hline & & 0.36481 & -11.67498 \\
\hline & & 0.3651 & -12.34604 \\
\hline & & 0.36714 & -13.19592 \\
\hline & & 0.37017 & -14.51011 \\
\hline & & 0.37358 & -15.74857 \\
\hline & & 0.37656 & -17.03803 \\
\hline & & 0.37885 & -18.02927 \\
\hline & & 0.37983 & -18.75309 \\
\hline & & 0.38219 & -19.75552 \\
\hline & & 0.38304 & -20.64565 \\
\hline & & 0.38539 & -21.87217 \\
\hline & & 0.38725 & -22.92315 \\
\hline & & 0.38808 & -23.74243 \\
\hline & & 0.38926 & -24.47624 \\
\hline & & 0.39119 & -25.36271 \\
\hline & & 0.39211 & -26.30611 \\
\hline & & 0.39336 & -26.99131 \\
\hline & & 0.39422 & -27.72121 \\
\hline & & 0.39628 & -28.6632 \\
\hline
\end{tabular}

Table S10: IV for ternary 3D chalcogenides. Here, data for $\mathrm{Cu}_{2} \mathrm{SnS}_{3}$ were provided by corresponding author(s) of $\mathrm{ref}^{8}$, data for $\mathrm{Cu}_{2}(\mathrm{Sn}, \mathrm{Ge}) \mathrm{S}_{3}$ were obtained by tracing Figure $5 \mathrm{a}$ in ref ${ }^{9}$, data for $\mathrm{Cu}_{2} \mathrm{GeS}_{3}$ were obtained by tracing Figure $8 \mathrm{a}$ in $\mathrm{ref}^{10}$, and data for $\mathrm{AgBiS}_{2}$ were provided by the corresponding author(s) of ref ${ }^{11}$.

\begin{tabular}{|c|c|c|c|c|c|c|c|}
\hline \multicolumn{2}{|c|}{$\mathrm{Cu}_{2} \mathrm{SnS}_{3}$} & \multicolumn{2}{|c|}{$\mathrm{Cu}_{2}(\mathrm{Sn}, \mathrm{Ge}) \mathrm{S}_{3}$} & \multicolumn{2}{|c|}{$\mathrm{Cu}_{2} \mathrm{GeS}_{3}$} & \multicolumn{2}{|c|}{$\mathrm{AgBiS}_{2}$} \\
\hline $\mathrm{V}(\mathrm{V})$ & $\mathrm{J}\left(\mathrm{mA} \mathrm{cm^{-2 } )}\right.$ & $\mathrm{V}(\mathrm{V})$ & $\mathrm{J}\left(\mathrm{mA} \mathrm{cm}^{-2}\right)$ & $\mathrm{V}(\mathrm{V})$ & $\mathrm{J}\left(\mathrm{mA} \mathrm{cm}^{-2}\right)$ & $\mathrm{V}(\mathrm{V})$ & $\mathrm{J}\left(\mathrm{mA} \mathrm{cm} \mathrm{c}^{-2}\right)$ \\
\hline-0.3 & 39.4 & -0.09726 & 27.05619 & $\frac{1.14199}{-0.19}$ & 17.20556 & -0.102 & 22.26805 \\
\hline-0.295 & 39.3 & -0.08906 & 27.05619 & -0.1289 & 17.00277 & -0.093 & 22.23905 \\
\hline-0.29 & 39.3 & -0.08086 & 27.00042 & -0.1158 & 16.81477 & -0.084 & 22.22781 \\
\hline-0.285 & 39.2 & -0.07265 & 26.96059 & -0.10271 & 16.6141 & -0.075 & 22.20888 \\
\hline-0.28 & 39.1 & -0.06445 & 26.92873 & -0.08962 & 16.41765 & -0.066 & 22.1929 \\
\hline-0.275 & 39 & -0.05625 & 26.865 & -0.07653 & 16.20641 & -0.057 & 22.19053 \\
\hline-0.27 & 38.9 & $\begin{array}{l}-0.04805 \\
\end{array}$ & 26.865 & $\begin{array}{l}-0.06343 \\
\end{array}$ & 16.02686 & -0.048 & 22.17692 \\
\hline-0.265 & 38.8 & $\begin{array}{r}-0.03985 \\
\end{array}$ & 26.865 & $\begin{array}{r}-0.05034 \\
\end{array}$ & 15.80506 & -0.039 & 22.15148 \\
\hline
\end{tabular}




\begin{tabular}{|c|c|c|c|c|c|c|c|}
\hline-0.26 & 38.8 & -0.03165 & 26.85703 & -0.03725 & 15.59383 & -0.03 & 22.1355 \\
\hline-0.255 & 38.7 & -0.02344 & 26.7694 & -0.02416 & 15.38048 & -0.021 & 22.11775 \\
\hline-0.25 & 38.6 & -0.01524 & 26.69771 & -0.01106 & 15.15446 & -0.012 & 22.10888 \\
\hline-0.245 & 38.5 & -0.00704 & 26.67381 & 0.00203 & 14.9447 & -0.003 & 22.08402 \\
\hline-0.24 & 38.4 & $8.7 \mathrm{E}-4$ & 26.69429 & 0.01512 & 14.71508 & 0.006 & 22.0716 \\
\hline-0.235 & 38.4 & 0.00936 & 26.57821 & 0.02821 & 14.47639 & 0.015 & 22.05148 \\
\hline-0.23 & 38.3 & 0.01756 & 26.55431 & 0.0413 & 14.26304 & 0.024 & 22.02367 \\
\hline-0.225 & 38.2 & 0.02576 & 26.50651 & 0.0544 & 14.02857 & 0.033 & 22.02071 \\
\hline-0.22 & 38.1 & 0.03397 & 26.48261 & 0.06749 & 13.75607 & 0.042 & 21.99349 \\
\hline-0.215 & 38 & 0.04217 & 26.41888 & 0.08058 & 13.51104 & 0.051 & 21.97633 \\
\hline-0.21 & 37.9 & 0.05037 & 26.37905 & 0.09367 & 13.26811 & 0.06 & 21.95385 \\
\hline-0.205 & 37.9 & 0.05857 & 26.31532 & 0.10677 & 13.00407 & 0.069 & 21.93018 \\
\hline-0.2 & 37.8 & 0.06677 & 26.27549 & 0.11986 & 12.76537 & 0.078 & 21.90473 \\
\hline-0.195 & 37.7 & 0.07497 & 26.20379 & 0.13295 & 12.50555 & 0.087 & 21.89053 \\
\hline-0.19 & 37.6 & 0.08317 & 26.14803 & 0.14604 & 12.24996 & 0.096 & 21.85207 \\
\hline-0.185 & 37.6 & 0.09138 & 26.10023 & 0.15914 & 11.98169 & 0.105 & 21.82071 \\
\hline-0.18 & 37.5 & 0.09958 & 26.02854 & 0.17223 & 11.70497 & 0.114 & 21.79882 \\
\hline-0.175 & 37.4 & 0.10778 & 25.94887 & 0.18532 & 11.44514 & 0.123 & 21.77278 \\
\hline-0.17 & 37.3 & 0.11598 & 25.90904 & 0.19841 & 11.15364 & 0.132 & 21.73432 \\
\hline-0.165 & 37.2 & 0.12418 & 25.81344 & 0.21151 & 10.86636 & 0.141 & 21.68994 \\
\hline-0.16 & 37.2 & 0.13238 & 25.73378 & 0.2246 & 10.57696 & 0.15 & 21.65089 \\
\hline-0.155 & 37.1 & 0.14059 & 25.66208 & 0.23769 & 10.28123 & 0.159 & 21.6142 \\
\hline-0.15 & 37 & 0.14879 & 25.55852 & 0.25078 & 9.9686 & 0.168 & 21.56036 \\
\hline-0.145 & 36.9 & 0.15699 & 25.47886 & 0.26388 & 9.68343 & 0.177 & 21.50769 \\
\hline-0.14 & 36.8 & 0.16519 & 25.36733 & 0.27697 & 9.37714 & 0.186 & 21.44379 \\
\hline-0.135 & 36.8 & 0.17339 & 25.3275 & 0.29006 & 9.0624 & 0.195 & 21.38817 \\
\hline-0.13 & 36.7 & 0.18159 & 25.2319 & 0.30315 & 8.74132 & 0.204 & 21.32367 \\
\hline-0.125 & 36.6 & 0.18979 & 25.08054 & 0.31625 & 8.41813 & 0.213 & 21.2432 \\
\hline-0.12 & 36.5 & 0.198 & 24.94512 & 0.32934 & 8.08015 & 0.222 & 21.16805 \\
\hline-0.115 & 36.4 & 0.2062 & 24.85749 & 0.34243 & 7.75696 & 0.231 & 21.07041 \\
\hline-0.11 & 36.4 & 0.2144 & 24.69816 & 0.35552 & 7.42109 & 0.24 & 20.97811 \\
\hline-0.105 & 36.3 & 0.2226 & 24.5229 & 0.36861 & 7.08523 & 0.249 & 20.85503 \\
\hline-0.1 & 36.2 & 0.2308 & 24.36358 & 0.38171 & 6.73246 & 0.258 & 20.73905 \\
\hline-0.095 & 36.1 & 0.239 & 24.18832 & 0.3948 & 6.37758 & 0.267 & 20.59941 \\
\hline-0.09 & 36 & 0.24721 & 23.98916 & 0.40789 & 6.00158 & 0.276 & 20.45089 \\
\hline-0.085 & 36 & 0.25541 & 23.7422 & 0.42098 & 5.64671 & 0.285 & 20.27456 \\
\hline-0.08 & 35.9 & 0.26361 & 23.50322 & 0.43408 & 5.26225 & 0.294 & 20.08225 \\
\hline-0.075 & 35.8 & 0.27181 & 23.26423 & 0.44717 & 4.88203 & 0.303 & 19.85385 \\
\hline-0.07 & 35.7 & 0.28001 & 22.96151 & 0.46026 & 4.48913 & 0.312 & 19.59349 \\
\hline-0.065 & 35.6 & 0.28821 & 22.63489 & 0.47335 & 4.08778 & 0.321 & 19.30592 \\
\hline-0.06 & 35.5 & 0.29641 & 22.2525 & 0.48645 & 3.68854 & 0.33 & 18.96095 \\
\hline-0.055 & 35.5 & 0.30427 & 21.84622 & 0.49954 & 3.27663 & 0.339 & 18.56746 \\
\hline-0.05 & 35.4 & 0.30872 & 21.49571 & 0.51263 & 2.8436 & 0.348 & 18.10355 \\
\hline-0.045 & 35.3 & 0.31662 & 21.10194 & 0.52572 & 2.41479 & 0.357 & 17.56036 \\
\hline-0.04 & 35.2 & 0.32483 & 20.62396 & 0.53882 & 1.94796 & 0.366 & 16.90178 \\
\hline-0.035 & 35.1 & 0.32815 & 20.19378 & 0.55191 & 1.51281 & 0.375 & 16.15089 \\
\hline-0.03 & 35 & 0.33476 & 19.78029 & 0.565 & 1.05654 & 0.384 & 15.20178 \\
\hline-0.025 & 35 & 0.34129 & 19.20672 & 0.5775 & 0.58189 & 0.393 & 14.10178 \\
\hline-0.02 & 34.9 & 0.34699 & 18.68559 & 0.59 & 0.11593 & 0.402 & 12.77396 \\
\hline-0.015 & 34.8 & 0.35246 & 18.10604 & 0.60309 & -0.37987 & 0.411 & 11.16568 \\
\hline-0.01 & 34.7 & 0.35793 & 17.53247 & 0.61559 & -0.89069 & 0.42 & 9.27456 \\
\hline-0.005 & 34.6 & 0.36339 & 16.9111 & 0.62808 & -1.36634 & 0.429 & 6.93846 \\
\hline 0 & 34.5 & 0.36876 & 16.24022 & 0.63998 & -1.84679 & 0.438 & 4.23905 \\
\hline 0.005 & 34.4 & 0.37296 & 15.69623 & 0.65129 & -2.35514 & 0.447 & 0.93787 \\
\hline 0.01 & 34.3 & 0.37677 & 15.08624 & 0.6626 & -2.85626 & 0.456 & -3.01302 \\
\hline 0.015 & 34.3 & 0.38048 & 14.50447 & 0.67331 & -3.33389 & 0.465 & -7.70651 \\
\hline 0.02 & 34.2 & 0.38458 & 13.90381 & 0.68343 & -3.8254 & 0.474 & -13.20414 \\
\hline 0.025 & 34.1 & 0.38829 & 13.20847 & 0.69354 & -4.34595 & 0.483 & -19.62959 \\
\hline 0.03 & 34 & 0.39073 & 12.6531 & 0.69997 & -4.65318 & 0.492 & -27.1284 \\
\hline 0.035 & 33.9 & 0.3946 & 11.98924 & & & 0.501 & -35.81716 \\
\hline 0.04 & 33.8 & 0.39876 & 11.27095 & & & 0.51 & -45.52367 \\
\hline 0.045 & 33.7 & 0.40167 & 10.62966 & & & 0.519 & -56.88876 \\
\hline 0.05 & 33.6 & 0.4044 & 10.05609 & & & 0.528 & -69.53846 \\
\hline 0.055 & 33.4 & 0.40713 & 9.48251 & & & 0.537 & -83.32722 \\
\hline 0.06 & 33.3 & 0.40987 & 8.87707 & & & 0.546 & -98.24556 \\
\hline 0.065 & 33.2 & 0.4126 & 8.2318 & & & 0.555 & -115.07219 \\
\hline 0.07 & 33.1 & 0.41534 & 7.5945 & & & 0.564 & -132.9574 \\
\hline 0.075 & 33 & 0.41807 & 6.95719 & & & 0.573 & -152.30059 \\
\hline 0.08 & 32.9 & 0.42012 & 6.33104 & & & 0.582 & -172.58994 \\
\hline 0.085 & 32.7 & 0.42266 & 5.67006 & & & 0.591 & -193.67633 \\
\hline 0.09 & 32.6 & 0.42422 & 5.0883 & & & 0.6 & -216.86864 \\
\hline 0.095 & 32.4 & 0.42741 & 4.37345 & & & & \\
\hline 0.1 & 32.3 & 0.4303 & 3.51309 & & & & \\
\hline 0.105 & 32.1 & 0.43219 & 2.78285 & & & & \\
\hline 0.11 & 32 & 0.43535 & 2.11937 & & & & \\
\hline 0.115 & 31.8 & 0.43721 & 1.45247 & & & & \\
\hline 0.12 & 31.6 & 0.43906 & 0.76737 & & & & \\
\hline 0.125 & 31.4 & 0.44245 & -0.10361 & & & & \\
\hline 0.13 & 31.2 & 0.44492 & -0.98066 & & & & \\
\hline 0.135 & 31 & 0.44729 & -1.88541 & & & & \\
\hline 0.14 & 30.7 & 0.44951 & -2.54661 & & & & \\
\hline 0.145 & 30.5 & 0.45136 & -3.07011 & & & & \\
\hline
\end{tabular}




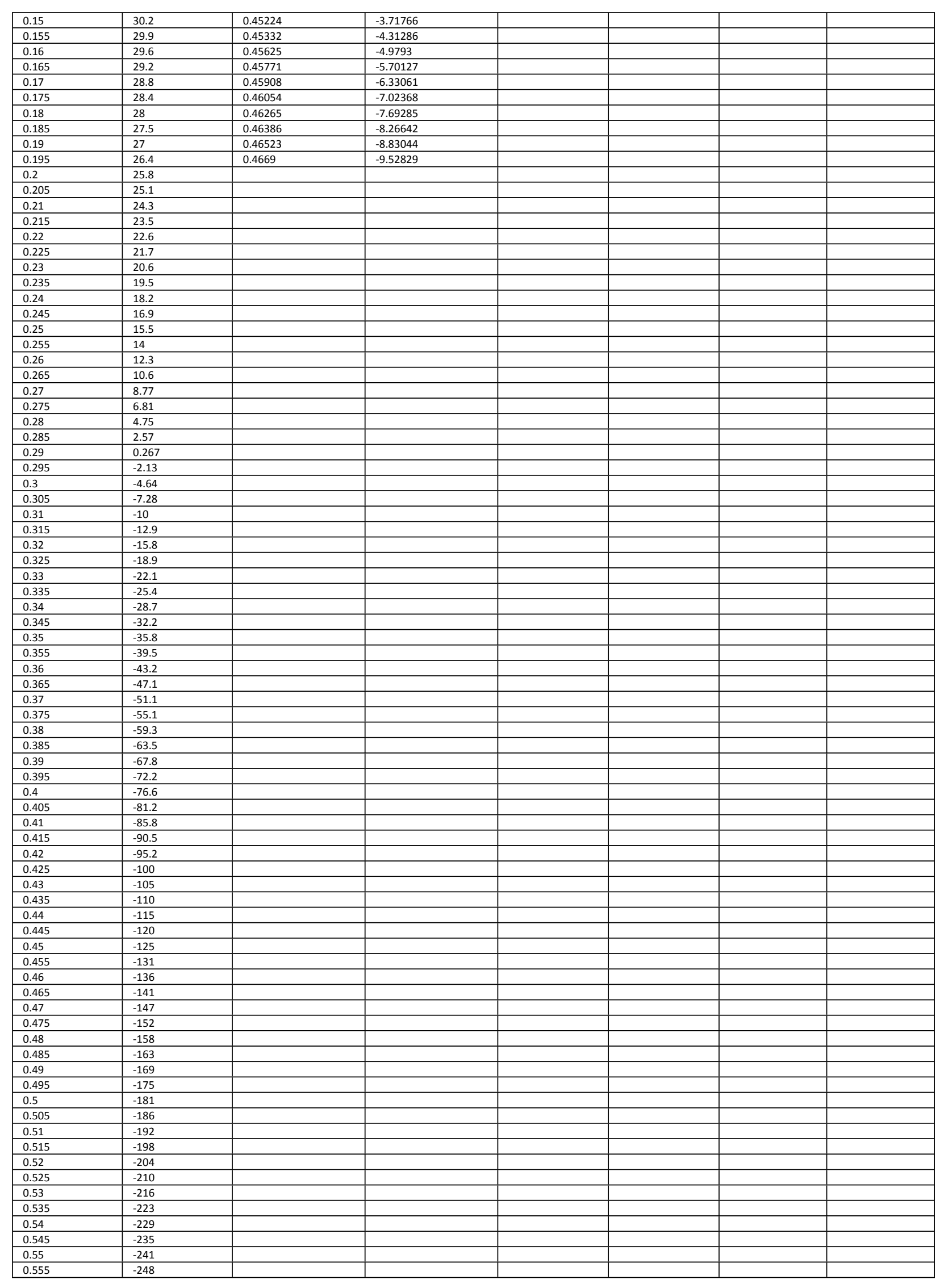




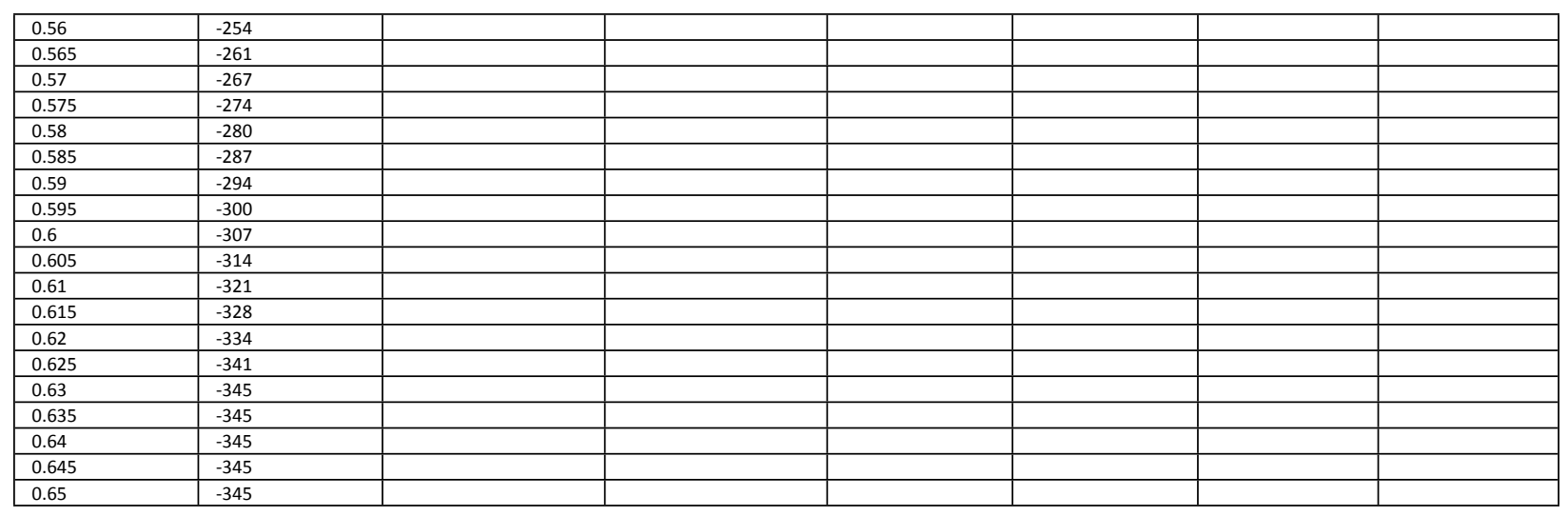

Table S11: IV data for kesterites. Here, data for $\mathrm{Cu}_{2} \mathrm{ZnSnS}_{4}$ were provided by the corresponding author(s) of ref ${ }^{12}$, data for $\mathrm{Cu}_{2} \mathrm{ZnSn}(\mathrm{S}, \mathrm{Se})_{4}$-IBM were provided by the corresponding author(s) of ref ${ }^{13}$, data for $\mathrm{Cu}_{2} \mathrm{ZnSn}(\mathrm{S}, \mathrm{Se})_{4}$-DGIST were provided by the corresponding author(s) of ref ${ }^{14}$, and data for $\mathrm{Cu}_{2} \mathrm{ZnSnSe}_{4}$ were obtained by tracing Figure $2 b$ in ref $^{15}$.

\begin{tabular}{|c|c|c|c|c|c|c|c|}
\hline \multicolumn{2}{|c|}{$\mathrm{Cu}_{2} \mathrm{ZnSnS}_{4}$} & \multicolumn{2}{|c|}{$\mathrm{Cu}_{2} \mathrm{ZnSn}(\mathrm{S}, \mathrm{Se})_{4}-\mathrm{IBM}$} & \multicolumn{2}{|c|}{$\mathrm{Cu}_{2} \mathrm{ZnSn}(\mathrm{S}, \mathrm{Se})_{4}$-DGIST } & \multicolumn{2}{|c|}{$\mathrm{Cu}_{2} \mathrm{ZnSnSe} \mathrm{Se}_{4}$} \\
\hline $\mathrm{V}(\mathrm{V})$ & $\mathrm{J}\left(\mathrm{mA} \mathrm{cm}^{-2}\right)$ & $\mathrm{V}(\mathrm{V})$ & $\mathrm{J}\left(\mathrm{mA} \mathrm{cm} \mathrm{cm}^{-2}\right)$ & $\mathrm{V}(\mathrm{V})$ & $\mathrm{J}\left(\mathrm{mA} \mathrm{cm} \mathrm{cm}^{-2}\right)$ & $\mathrm{V}(\mathrm{V})$ & $\mathrm{J}\left(\mathrm{mA} \mathrm{cm} \mathrm{cm}^{-2}\right)$ \\
\hline-0.13719 & 21.80847 & -0.1 & 35.43835 & -0.1 & 35.47188 & -0.00542 & 37.43524 \\
\hline-0.1091 & 21.7982 & -0.08915 & 35.41934 & -0.095 & 35.46341 & -0.00242 & 37.4352 \\
\hline-0.08076 & 21.77982 & -0.0783 & 35.40746 & -0.09 & 35.47292 & 0.00272 & 37.43272 \\
\hline-0.0528 & 21.77383 & -0.06745 & 35.38133 & -0.085 & 35.45939 & 0.01059 & 37.4352 \\
\hline-0.02435 & 21.74989 & -0.0566 & 35.3837 & -0.08 & 35.46674 & 0.01724 & 37.42528 \\
\hline 0.00432 & 21.73578 & -0.04575 & 35.36232 & -0.07 & 35.45562 & 0.07713 & 37.32606 \\
\hline 0.03238 & 21.71783 & -0.0349 & 35.34331 & -0.065 & 35.451 & 0.08379 & 37.32606 \\
\hline 0.0607 & 21.69645 & -0.02405 & 35.32431 & -0.06 & 35.43801 & 0.11706 & 37.2442 \\
\hline 0.08886 & 21.68619 & -0.0132 & 35.31243 & -0.055 & 35.44373 & 0.12371 & 37.2318 \\
\hline 0.11921 & 21.65968 & -0.00235 & 35.30055 & -0.05 & 35.4326 & 0.15033 & 37.13506 \\
\hline 0.14668 & 21.63104 & 0.00845 & 35.28154 & -0.04 & 35.42096 & 0.15699 & 37.13506 \\
\hline 0.17566 & 21.61052 & 0.0193 & 35.26016 & -0.035 & 35.4275 & 0.16364 & 37.13506 \\
\hline 0.20353 & 21.58016 & 0.03015 & 35.23165 & -0.03 & 35.41655 & 0.1703 & 37.13506 \\
\hline 0.23164 & 21.54895 & 0.041 & 35.22452 & -0.025 & 35.41682 & 0.17695 & 37.10778 \\
\hline 0.25962 & 21.51304 & 0.05185 & 35.19838 & -0.02 & 35.41205 & 0.21022 & 36.91678 \\
\hline 0.2879 & 21.4767 & 0.0627 & 35.17463 & -0.01 & 35.41139 & 0.21688 & 36.91678 \\
\hline 0.31607 & 21.43181 & 0.07355 & 35.15799 & -0.005 & 35.40117 & 0.22353 & 36.87957 \\
\hline 0.34409 & 21.36298 & 0.0844 & 35.13424 & 0 & 35.41676 & 0.23019 & 36.83492 \\
\hline 0.37431 & 21.30184 & 0.09525 & 35.11523 & 0.005 & 35.401 & 0.23684 & 36.81756 \\
\hline 0.40183 & 21.20735 & 0.1061 & 35.10097 & 0.01 & 35.40373 & 0.2435 & 36.80764 \\
\hline 0.4301 & 21.09748 & 0.11695 & 35.07484 & 0.015 & 35.41066 & 0.25015 & 36.80764 \\
\hline 0.45955 & 20.93801 & 0.1278 & 35.06058 & 0.02 & 35.40291 & 0.26346 & 36.66129 \\
\hline 0.48675 & 20.72809 & 0.13865 & 35.01782 & 0.025 & 35.38936 & 0.27011 & 36.61664 \\
\hline 0.51434 & 20.42967 & 0.1495 & 35.00594 & 0.035 & 35.38091 & 0.27677 & 36.53975 \\
\hline 0.54118 & 19.99829 & 0.16035 & 34.98693 & 0.04 & 35.37646 & 0.28342 & 36.41077 \\
\hline 0.56771 & 19.36298 & 0.1712 & 34.95842 & 0.05 & 35.37937 & 0.29008 & 36.31651 \\
\hline 0.59003 & 18.56947 & 0.18205 & 34.92516 & 0.055 & 35.3643 & 0.29673 & 36.22225 \\
\hline 0.61037 & 17.5481 & 0.1929 & 34.88952 & 0.065 & 35.35612 & 0.30339 & 36.12551 \\
\hline 0.62703 & 16.42411 & 0.20375 & 34.87052 & 0.07 & 35.35202 & 0.31004 & 36.01637 \\
\hline 0.64227 & 15.11586 & 0.2146 & 34.83963 & 0.075 & 35.34557 & 0.3167 & 35.92955 \\
\hline 0.65514 & 13.75417 & 0.2254 & 34.81112 & 0.08 & 35.35004 & 0.32335 & 35.80801 \\
\hline 0.66617 & 12.37922 & 0.23625 & 34.77073 & 0.085 & 35.35108 & 0.33001 & 35.61454 \\
\hline 0.67583 & 10.98846 & 0.2471 & 34.73272 & 0.09 & 35.33714 & 0.33666 & 35.4037 \\
\hline 0.68531 & 9.45832 & 0.25795 & 34.69233 & 0.095 & 35.33853 & 0.34332 & 35.25487 \\
\hline 0.69437 & 7.82471 & 0.2688 & 34.63768 & 0.1 & 35.33526 & 0.34997 & 34.9969 \\
\hline 0.70177 & 6.32322 & 0.27965 & 34.58304 & 0.11 & 35.31888 & 0.35662 & 34.64963 \\
\hline 0.70942 & 4.69859 & 0.2905 & 34.52126 & 0.115 & 35.31626 & 0.36328 & 34.2602 \\
\hline 0.71667 & 3.00004 & 0.30135 & 34.44048 & 0.125 & 35.31755 & 0.36993 & 33.89805 \\
\hline 0.72263 & 1.49282 & 0.3122 & 34.34783 & 0.13 & 35.31176 & 0.37629 & 33.5116 \\
\hline 0.72623 & 0.56789 & 0.32305 & 34.21953 & 0.135 & 35.29255 & 0.38234 & 32.93042 \\
\hline 0.72789 & 0.12044 & 0.3339 & 34.07698 & 0.14 & 35.29838 & 0.38869 & 32.33536 \\
\hline \multirow[t]{7}{*}{0.72857} & -0.14477 & 0.34475 & 33.88928 & 0.145 & 35.28707 & 0.39474 & 31.74776 \\
\hline & & 0.3556 & 33.64932 & 0.15 & 35.27654 & 0.39988 & 31.08799 \\
\hline & & 0.36645 & 33.34046 & 0.155 & 35.27873 & 0.40442 & 30.47463 \\
\hline & & 0.3773 & 32.92231 & 0.16 & 35.27475 & 0.40865 & 29.81095 \\
\hline & & 0.38815 & 32.37349 & 0.17 & 35.25414 & 0.41228 & 29.22783 \\
\hline & & 0.399 & 31.67498 & 0.175 & 35.24276 & 0.41531 & 28.67667 \\
\hline & & 0.40985 & 30.73414 & 0.18 & 35.21334 & 0.41863 & 28.08095 \\
\hline
\end{tabular}




\begin{tabular}{|c|c|c|c|c|c|c|c|}
\hline & & 0.4207 & 29.55096 & 0.185 & 35.23191 & 0.42196 & 27.43247 \\
\hline & & 0.43155 & 28.02328 & 0.19 & 35.21443 & 0.42529 & 26.73943 \\
\hline & & 0.44235 & 26.10359 & 0.195 & 35.20375 & 0.42861 & 25.99181 \\
\hline & & 0.4532 & 23.74436 & 0.2 & 35.18585 & 0.43134 & 25.36835 \\
\hline & & 0.46405 & 20.83868 & 0.205 & 35.16245 & 0.43376 & 24.7749 \\
\hline & & 0.4749 & 17.37467 & 0.215 & 35.14111 & 0.43618 & 24.18486 \\
\hline & & 0.48575 & 13.30007 & 0.22 & 35.12596 & 0.4386 & 23.53342 \\
\hline & & 0.4966 & 8.57923 & 0.225 & 35.09478 & 0.44023 & 22.93588 \\
\hline & & 0.50745 & 3.25731 & 0.23 & 35.09958 & 0.44244 & 22.22958 \\
\hline & & 0.5183 & -2.67522 & 0.235 & 35.08828 & 0.4446 & 21.54746 \\
\hline & & 0.52915 & -9.21121 & 0.24 & 35.04398 & 0.44688 & 20.82401 \\
\hline & & 0.54 & -16.24852 & 0.245 & 35.04042 & 0.44918 & 20.08186 \\
\hline & & & & 0.25 & 35.01218 & 0.4513 & 19.31787 \\
\hline & & & & 0.26 & 34.97344 & 0.4535 & 18.56558 \\
\hline & & & & 0.265 & 34.95154 & 0.45584 & 17.73533 \\
\hline & & & & 0.27 & 34.92727 & 0.45796 & 16.81446 \\
\hline & & & & 0.275 & 34.87475 & 0.46038 & 15.88677 \\
\hline & & & & 0.28 & 34.84278 & 0.46258 & 14.96005 \\
\hline & & & & 0.285 & 34.76796 & 0.46491 & 13.96635 \\
\hline & & & & 0.29 & 34.75479 & 0.46649 & 13.10778 \\
\hline & & & & 0.295 & 34.7035 & 0.46846 & 12.39914 \\
\hline & & & & 0.3 & 34.65687 & 0.47005 & 11.65757 \\
\hline & & & & 0.305 & 34.60995 & 0.47157 & 10.87616 \\
\hline & & & & 0.31 & 34.53747 & 0.47308 & 10.12278 \\
\hline & & & & 0.315 & 34.50883 & 0.47429 & 9.53615 \\
\hline & & & & 0.32 & 34.39101 & 0.4755 & 8.99386 \\
\hline & & & & 0.325 & 34.322 & 0.47671 & 8.44475 \\
\hline & & & & 0.33 & 34.21261 & 0.47792 & 7.8513 \\
\hline & & & & 0.335 & 34.12823 & 0.47913 & 7.27285 \\
\hline & & & & 0.34 & 34.02057 & 0.48034 & 6.56207 \\
\hline & & & & 0.345 & 33.87246 & 0.48155 & 5.90723 \\
\hline & & & & 0.35 & 33.77423 & 0.48246 & 5.40246 \\
\hline & & & & 0.355 & 33.63591 & 0.48367 & 4.92951 \\
\hline & & & & 0.36 & 33.49338 & 0.48437 & 4.21555 \\
\hline & & & & 0.365 & 33.30706 & 0.48558 & 3.5698 \\
\hline & & & & 0.37 & 33.12623 & 0.48679 & 2.88313 \\
\hline & & & & 0.375 & 32.94925 & 0.48778 & 2.20464 \\
\hline & & & & 0.38 & 32.70012 & 0.48899 & 1.5498 \\
\hline & & & & 0.385 & 32.45818 & 0.4902 & 0.8513 \\
\hline & & & & 0.39 & 32.15845 & 0.49136 & 0.14422 \\
\hline & & & & 0.395 & 31.89546 & 0.49188 & -0.09398 \\
\hline & & & & 0.4 & 31.57537 & 0.49214 & -0.27285 \\
\hline & & & & 0.405 & 31.21041 & 0.49272 & -0.66242 \\
\hline & & & & 0.41 & 30.83002 & & \\
\hline & & & & 0.415 & 30.40868 & & \\
\hline & & & & 0.42 & 29.98453 & & \\
\hline & & & & 0.425 & 29.46097 & & \\
\hline & & & & 0.43 & 28.92581 & & \\
\hline & & & & 0.435 & 28.33982 & & \\
\hline & & & & 0.44 & 27.70645 & & \\
\hline & & & & 0.445 & 27.02425 & & \\
\hline & & & & 0.45 & 26.27648 & & \\
\hline & & & & 0.455 & 25.49833 & & \\
\hline & & & & 0.46 & 24.6276 & & \\
\hline & & & & 0.465 & 23.70924 & & \\
\hline & & & & 0.47 & 22.73337 & & \\
\hline & & & & 0.475 & 21.6566 & & \\
\hline & & & & 0.48 & 20.53043 & & \\
\hline & & & & 0.485 & 19.33195 & & \\
\hline & & & & 0.49 & 18.05435 & & \\
\hline & & & & 0.495 & 16.68768 & & \\
\hline & & & & 0.5 & 15.25448 & & \\
\hline & & & & 0.505 & 13.74228 & & \\
\hline & & & & 0.51 & 12.13674 & & \\
\hline & & & & 0.515 & 10.43882 & & \\
\hline & & & & 0.52 & 8.68353 & & \\
\hline & & & & 0.525 & 6.81649 & & \\
\hline & & & & 0.53 & 4.87775 & & \\
\hline & & & & 0.535 & 2.85939 & & \\
\hline & & & & 0.54 & 0.64201 & & \\
\hline & & & & 0.545 & -1.58333 & & \\
\hline & & & & 0.55 & -3.9914 & & \\
\hline & & & & 0.555 & -6.49228 & & \\
\hline & & & & 0.56 & -9.14521 & & \\
\hline
\end{tabular}

Table S12: IV data for kesterite-inspired chalcogenides. Here, data for $\mathrm{Cu}_{2} \mathrm{BaSn}(\mathrm{S}, \mathrm{Se})_{4}$ were provided by the corresponding author(s) of $\operatorname{ref}^{16}$, data for $\mathrm{Cu}_{2}(\mathrm{Zn}, \mathrm{Cd}) \mathrm{SnS}_{4}$ were obtained by tracing Figure $1 \mathrm{c}$ in $\mathrm{ref}^{17}$, data for $\mathrm{Cu}_{2} \mathrm{CdSnS}_{4}$ were provided by the corresponding author(s) of ref ${ }^{18}$, data for $\mathrm{Ag}_{2} \mathrm{ZnSnSe}{ }_{4}$ were 
obtained by tracing Figure $3 \mathrm{~b}$ in $\mathrm{ref}^{19}$, and data for $\mathrm{Cu}_{2} \mathrm{ZnGeSe}_{4}$ were provided by the corresponding author(s) of ref ${ }^{20}$.

\begin{tabular}{|c|c|c|c|c|c|c|c|c|c|}
\hline \multicolumn{2}{|c|}{$\mathrm{Cu}_{2} \mathrm{BaSn}(\mathrm{S}, \mathrm{Se})_{4}$} & \multicolumn{2}{|c|}{$\mathrm{Cu}_{2}(\mathrm{Zn}, \mathrm{Cd}) \mathrm{SnS}_{4}$} & \multicolumn{2}{|c|}{$\mathrm{Cu}_{2} \mathrm{CdSnS}_{4}$} & \multicolumn{2}{|c|}{$\mathrm{Ag}_{2} \mathrm{ZnSnSe} \mathrm{Se}_{4}$} & \multicolumn{2}{|c|}{$\mathrm{Cu}_{2} \mathrm{ZnGeSe}_{4}$} \\
\hline $\mathrm{V}(\mathrm{V})$ & $\mathrm{J}\left(\mathrm{mA} \mathrm{cm}^{-2}\right)$ & $\mathrm{V}(\mathrm{V})$ & $\mathrm{J}\left(\mathrm{mA} \mathrm{cm} \mathrm{cm}^{-2}\right)$ & $\mathrm{V}(\mathrm{V})$ & $\mathrm{J}\left(\mathrm{mA} \mathrm{cm}{ }^{-2}\right)$ & $\mathrm{V}(\mathrm{V})$ & $\mathrm{J}\left(\mathrm{mA} \mathrm{cm}^{-2}\right)$ & $\mathrm{V}(\mathrm{V})$ & $\mathrm{J}\left(\mathrm{mA} \mathrm{cm}^{-2}\right)$ \\
\hline-0.5 & 19.01111 & -0.00733 & 27.77465 & -0.1 & 22.54272 & -0.19458 & 22.18879 & -0.3 & 25.45369 \\
\hline-0.48 & 18.7441 & -0.00204 & 27.77365 & -0.0934 & 22.53968 & -0.1818 & 22.0935 & -0.295 & 25.39667 \\
\hline-0.46 & 18.70149 & 0.0057 & 27.761 & -0.0868 & 22.53728 & -0.1694 & 22.07429 & -0.29 & 25.46235 \\
\hline-0.44 & 18.76969 & 0.0139 & 27.7446 & -0.0802 & 22.53165 & -0.15653 & 22.04346 & -0.285 & 25.37522 \\
\hline-0.42 & 18.58248 & 0.02461 & 27.7446 & -0.0736 & 22.52133 & -0.14506 & 21.99501 & -0.28 & 25.47307 \\
\hline-0.4 & 18.78789 & 0.03533 & 27.69259 & $\begin{array}{l}-0.0669 \\
\end{array}$ & 22.50772 & -0.13219 & 21.89172 & -0.275 & 25.41944 \\
\hline-0.38 & 18.4893 & 0.04604 & 27.67699 & -0.0603 & 22.48715 & -0.11978 & 21.85329 & -0.27 & 25.31424 \\
\hline-0.36 & 18.66229 & 0.05675 & 27.65879 & -0.0537 & 22.46741 & -0.10691 & 21.81445 & -0.265 & 25.41342 \\
\hline-0.34 & 18.46349 & 0.06746 & 27.65879 & -0.0471 & 22.46658 & -0.09594 & 21.73512 & -0.26 & 25.32102 \\
\hline-0.32 & 18.41919 & 0.07818 & 27.64059 & -0.0405 & 22.47506 & -0.08257 & 21.65631 & -0.255 & 25.24254 \\
\hline-0.3 & 18.55519 & 0.08889 & 27.63019 & -0.0339 & 22.45791 & -0.07016 & 21.59385 & -0.25 & 25.3276 \\
\hline-0.28 & 18.2248 & 0.0996 & 27.58078 & -0.0273 & 22.44804 & -0.05729 & 21.55021 & -0.245 & 25.25327 \\
\hline-0.26 & 18.4401 & 0.11032 & 27.57298 & -0.0207 & 22.45443 & -0.04632 & 21.50079 & -0.24 & 25.31895 \\
\hline-0.24 & 18.16638 & $\begin{array}{l}0.12103 \\
\end{array}$ & 27.57298 & -0.014 & 22.44392 & -0.03295 & 21.38246 & -0.235 & 25.23915 \\
\hline-0.22 & 18.21387 & 0.13174 & 27.57298 & -0.0074 & 22.42684 & -0.02054 & 21.28637 & -0.23 & 25.21243 \\
\hline-0.2 & 18.23118 & 0.14245 & 27.57298 & $-8 \mathrm{E}-4$ & 22.41968 & -0.00814 & 21.19989 & -0.225 & 25.18759 \\
\hline-0.16 & 18.09709 & 0.16388 & 27.47937 & 0.0124 & 22.37285 & 0.01667 & 21.03654 & -0.215 & 25.23182 \\
\hline-0.14 & 17.92912 & 0.17459 & 27.45857 & 0.019 & 22.34101 & 0.02908 & 20.95967 & -0.21 & 25.16012 \\
\hline-0.12 & 17.92979 & 0.1853 & 27.41696 & 0.0256 & 22.32759 & 0.04148 & 20.83956 & -0.205 & 25.10309 \\
\hline-0.1 & 17.92304 & 0.19602 & 27.40136 & 0.0322 & 22.32797 & 0.05328 & 20.80244 & -0.2 & 25.16821 \\
\hline-0.08 & 17.73485 & 0.20673 & 27.40136 & 0.0388 & 22.3031 & 0.06629 & 20.68582 & -0.195 & 25.10121 \\
\hline-0.06 & 17.90052 & 0.21744 & 27.39356 & 0.0455 & 22.29354 & 0.0787 & 20.57532 & -0.19 & 25.12267 \\
\hline-0.04 & 17.56293 & 0.22815 & 27.38576 & 0.0521 & 22.29633 & 0.0911 & 20.46002 & -0.185 & 25.05417 \\
\hline-0.02 & 17.6638 & 0.23887 & 27.33895 & 0.0587 & 22.28342 & 0.10245 & 20.40083 & -0.18 & 25.14468 \\
\hline 0 & 17.55829 & 0.24958 & 27.29215 & 0.0653 & 22.26304 & 0.11591 & 20.24863 & -0.175 & 25.00674 \\
\hline 0.02 & 17.40679 & 0.26029 & 27.25314 & 0.0719 & 22.25766 & 0.12832 & 20.13813 & -0.17 & 24.97513 \\
\hline 0.04 & 17.42256 & 0.271 & 27.22974 & 0.0785 & 22.22816 & 0.14072 & 19.97958 & -0.165 & 25.03083 \\
\hline 0.06 & 17.13042 & 0.28172 & 27.22974 & 0.0851 & 22.19601 & 0.15237 & 19.8653 & -0.16 & 24.9565 \\
\hline 0.08 & 17.27639 & 0.29243 & 27.22714 & 0.0917 & 22.17513 & 0.16553 & 19.66249 & -0.155 & 24.91829 \\
\hline 0.1 & 16.9471 & 0.30314 & 27.17253 & 0.0983 & 22.15677 & 0.17794 & 19.49914 & -0.15 & 25.00392 \\
\hline 0.12 & 16.95929 & 0.31386 & 27.14913 & 0.105 & 22.15418 & 0.19034 & 19.29256 & -0.145 & 24.87068 \\
\hline 0.14 & 16.76518 & 0.32457 & 27.11012 & 0.1116 & 22.13329 & 0.20202 & 19.16793 & -0.14 & 24.85581 \\
\hline 0.16 & 16.534 & 0.33528 & 27.07372 & 0.1182 & 22.09108 & 0.21516 & 18.87458 & -0.135 & 24.93166 \\
\hline 0.18 & 16.56839 & 0.34599 & 27.05292 & 0.1248 & 22.06899 & 0.22756 & 18.64877 & -0.13 & 24.85581 \\
\hline 0.2 & 16.1741 & 0.35671 & 27.00611 & 0.1314 & 22.05538 & 0.23997 & 18.39894 & -0.125 & 24.90343 \\
\hline 0.26 & 15.5554 & 0.38884 & 26.83709 & 0.1512 & 22.0419 & 0.27719 & 17.49092 & -0.11 & 24.83718 \\
\hline 0.28 & 15.40534 & 0.39956 & 26.76168 & 0.1579 & 22.02032 & 0.2896 & 17.13059 & -0.105 & 24.73462 \\
\hline 0.3 & 14.96664 & 0.41027 & 26.70967 & 0.1645 & 22.01025 & 0.30157 & 16.79063 & -0.1 & 24.75532 \\
\hline 0.32 & 14.81416 & 0.42098 & 26.62386 & 0.1711 & 21.99285 & 0.31441 & 16.27541 & -0.095 & 24.80162 \\
\hline 0.34 & 14.26063 & 0.43169 & 26.53545 & 0.1777 & 21.98576 & 0.32682 & 15.75654 & -0.09 & 24.68494 \\
\hline 0.36 & 13.91344 & 0.44241 & 26.43404 & 0.1843 & 21.96424 & 0.33923 & 15.20884 & -0.085 & 24.72916 \\
\hline 0.38 & 13.47948 & 0.45312 & 26.28582 & 0.1909 & 21.92165 & 0.35056 & 14.73653 & -0.08 & 24.67892 \\
\hline 0.4 & 12.88991 & 0.46383 & 26.11333 & 0.1975 & 21.89519 & 0.36405 & 13.86842 & -0.075 & 24.63206 \\
\hline 0.42 & 12.48299 & 0.47454 & 25.91571 & 0.2041 & 21.8793 & 0.37647 & 13.0757 & -0.07 & 24.69115 \\
\hline 0.44 & 11.7176 & 0.48526 & 25.67475 & 0.2107 & 21.85342 & 0.38888 & 12.24455 & -0.065 & 24.57786 \\
\hline 0.46 & 11.09868 & 0.49597 & 25.39131 & 0.2174 & 21.82146 & 0.39969 & 11.48215 & -0.06 & 24.59517 \\
\hline 0.48 & 10.23104 & 0.50668 & 24.99953 & 0.224 & 21.79658 & 0.41258 & 10.18934 & -0.055 & 24.62942 \\
\hline 0.5 & 9.34941 & 0.5174 & 24.51587 & 0.2306 & 21.77323 & 0.42274 & 9.08541 & -0.05 & 24.55434 \\
\hline 0.52 & 8.27885 & 0.52762 & 23.94796 & 0.2372 & 21.74057 & 0.43346 & 7.88987 & -0.045 & 24.55227 \\
\hline 0.54 & 6.9158 & 0.53687 & 23.33648 & 0.2438 & 21.69766 & 0.44507 & 6.76387 & -0.04 & 24.62528 \\
\hline 0.56 & 5.47473 & 0.54418 & 22.76759 & 0.2504 & 21.66475 & 0.45194 & 5.96972 & -0.035 & 24.48132 \\
\hline 0.58 & 3.52132 & 0.55051 & 22.10971 & 0.257 & 21.6488 & 0.46338 & 4.55809 & -0.03 & 24.48602 \\
\hline 0.6 & 1.34692 & 0.55684 & 21.46232 & 0.2636 & 21.62905 & 0.47354 & 3.38369 & -0.025 & 24.52027 \\
\hline 0.62 & -1.15744 & 0.56219 & 20.85115 & 0.2702 & 21.59715 & 0.4837 & 2.20929 & -0.02 & 24.45253 \\
\hline 0.64 & -4.11324 & 0.56658 & 20.22188 & 0.2769 & 21.55443 & 0.4948 & 1.10053 & -0.015 & 24.41827 \\
\hline 0.66 & -7.42895 & 0.57047 & 19.59975 & 0.2835 & 21.51892 & 0.50056 & 0.23394 & -0.01 & 24.4546 \\
\hline 0.68 & -11.06461 & 0.57437 & 19.01338 & 0.2901 & 21.47424 & 0.50925 & -1.02692 & -0.005 & 24.33999 \\
\hline 0.7 & -14.96206 & 0.57826 & 18.4127 & 0.2967 & 21.40715 & 0.51701 & -2.37675 & 0 & 24.38082 \\
\hline 0.72 & -19.1544 & 0.58216 & 17.75625 & 0.3033 & 21.34557 & 0.52435 & -3.53437 & 0.005 & 24.39889 \\
\hline 0.74 & -23.53282 & 0.58543 & 16.98641 & 0.3099 & 21.28823 & 0.53226 & -4.81783 & 0.01 & 24.29633 \\
\hline 0.76 & -28.05384 & 0.58849 & 16.28889 & 0.3165 & 21.21247 & 0.54073 & -6.09373 & 0.015 & 24.32719 \\
\hline 0.78 & -32.71496 & 0.59141 & 15.62624 & 0.3231 & 21.13462 & 0.5492 & -7.45662 & 0.02 & 24.25493 \\
\hline \multirow[t]{8}{*}{0.8} & -37.36049 & 0.59433 & 14.97504 & 0.3298 & 21.06544 & 0.55711 & -9.02378 & 0.025 & 24.30517 \\
\hline & & 0.59725 & 14.32478 & 0.3364 & 20.98316 & 0.56317 & -10.4609 & 0.03 & 24.19188 \\
\hline & & 0.60018 & 13.65165 & 0.343 & 20.90006 & 0.56854 & -11.73673 & 0.035 & 24.22275 \\
\hline & & 0.60271 & 13.02078 & 0.3496 & 20.80032 & 0.57394 & -12.98747 & 0.04 & 24.16234 \\
\hline & & 0.60543 & 12.35019 & 0.3562 & 20.69203 & 0.57915 & -14.23762 & 0.045 & 24.23404 \\
\hline & & 0.60758 & 11.6065 & 0.3628 & 20.56044 & 0.58436 & -15.45958 & 0.05 & 24.11604 \\
\hline & & 0.6103 & 10.88569 & 0.3694 & 20.43627 & 0.5903 & -16.79306 & 0.055 & 24.12489 \\
\hline & & 0.61245 & 10.12484 & 0.376 & 20.28304 & 0.59554 & -18.09779 & 0.06 & 24.08123 \\
\hline
\end{tabular}




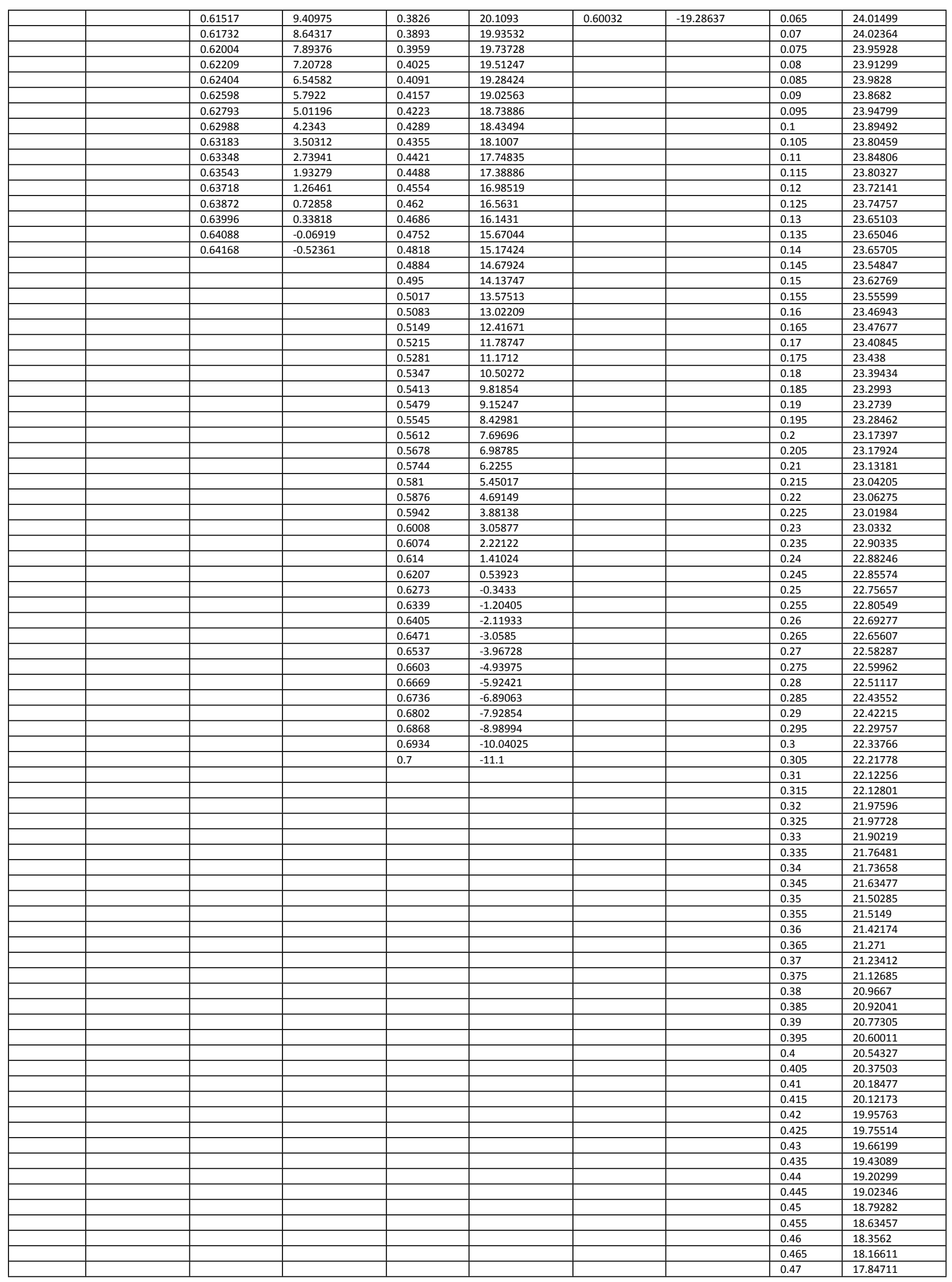




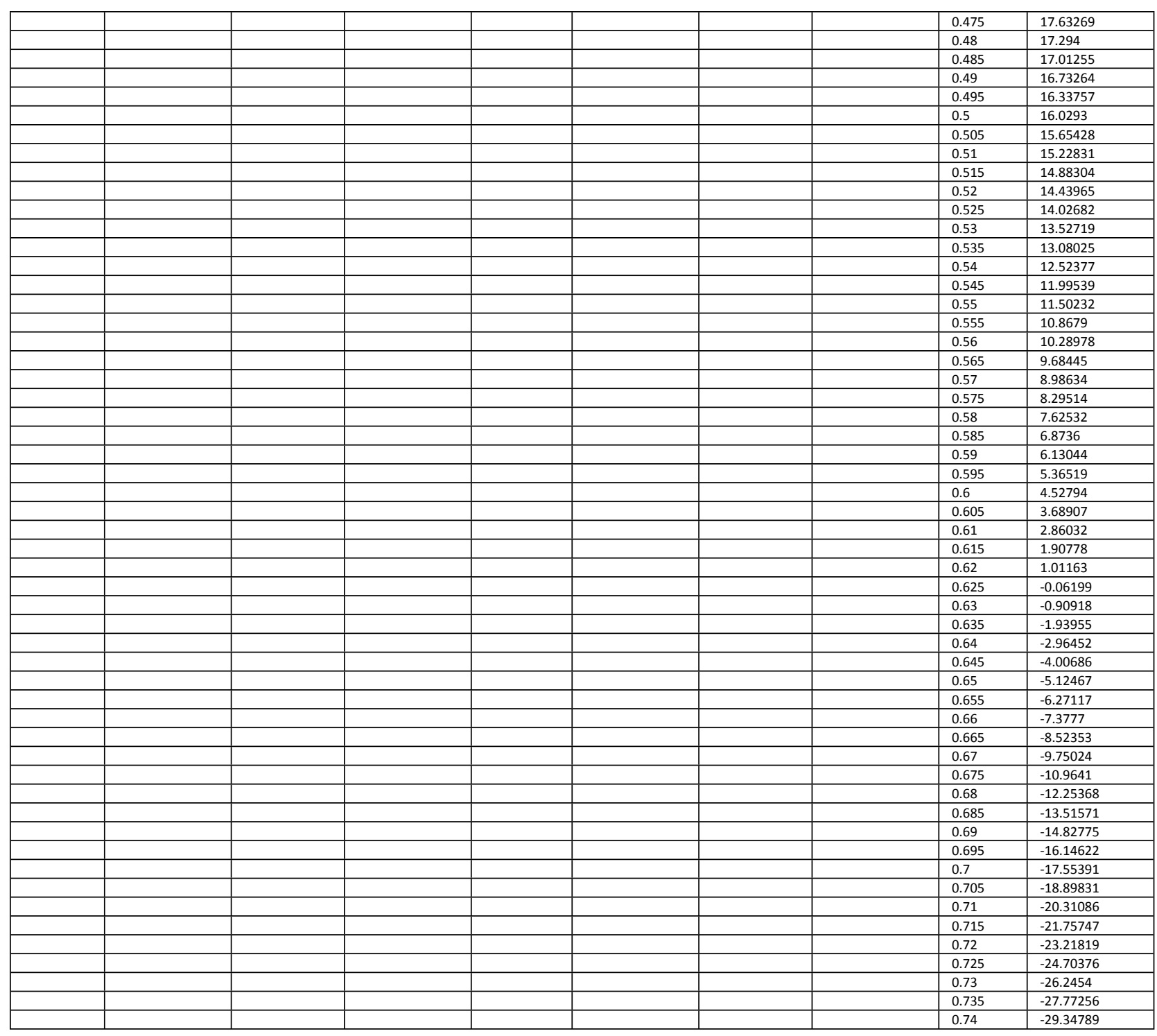


Table S13: Absorption coefficient data used to plot SLME values in Figure 12 in the main manuscript. Data were obtained from refs.

\begin{tabular}{|c|c|c|c|c|c|c|c|c|c|c|c|}
\hline \multicolumn{2}{|c|}{$\mathrm{Sb}_{2} \mathrm{Se}_{3}$} & \multicolumn{2}{|l|}{$\mathrm{Sb}_{2} \mathrm{~S}_{3}$} & \multicolumn{2}{|l|}{ SnS } & \multicolumn{2}{|l|}{ GeSe } & \multicolumn{2}{|c|}{$\mathrm{CuSbS}_{2}$} & CuSb & \\
\hline $\mathrm{eV}$ & $\alpha\left(\mathrm{cm}^{-1}\right)$ & $\mathrm{eV}$ & $\alpha\left(\mathrm{cm}^{-1}\right)$ & $\mathrm{eV}$ & $\alpha\left(\mathrm{cm}^{-1}\right)$ & $\mathrm{eV}$ & $\alpha\left(\mathrm{cm}^{-1}\right)$ & $\mathrm{eV}$ & $\alpha\left(\mathrm{cm}^{-1}\right)$ & $\mathrm{eV}$ & $\alpha\left(\mathrm{cm}^{-1}\right)$ \\
\hline 0.70132 & 4121.664 & 1.24004 & 2729.045 & 1.15013 & 42.34502 & 0.95749 & 13255.81 & 1.07191 & 1411.432 & 0.81989 & 2119.849 \\
\hline 0.71677 & 4792.055 & 1.25996 & 5774.854 & 1.15999 & 146.6367 & 0.96125 & 13310.08 & 1.08402 & 1591.527 & 0.83972 & 2046.896 \\
\hline 0.73292 & 5040.348 & 1.27987 & 7992.203 & 1.16985 & 266.5536 & 0.96496 & 13364.34 & 1.09594 & 1737.438 & 0.86001 & 1905.4 \\
\hline 0.74968 & 5363.128 & 1.30014 & 9429.825 & 1.18003 & 454.2937 & 0.96878 & 13472.87 & 1.10805 & 1861.407 & 0.87983 & 1905.4 \\
\hline 0.76736 & 5710.739 & 1.32006 & 9722.222 & 1.18989 & 664.8614 & 0.97254 & 13527.13 & 1.11997 & 1963.227 & 0.90012 & 1957.666 \\
\hline 0.78575 & 5909.373 & 1.33997 & 9137.427 & 1.20006 & 946.7843 & 0.97642 & 13563.31 & 1.13208 & 2019.368 & 0.91993 & 2133.39 \\
\hline 0.8052 & 6281.813 & 1.35989 & 7870.37 & 1.20992 & 1225.209 & 0.98024 & 13599.48 & 1.14401 & 2059.834 & 0.93975 & 2377.279 \\
\hline 0.82563 & 6554.935 & 1.38015 & 6067.251 & 1.2201 & 1607.337 & 0.98409 & 13653.75 & 1.15593 & 2068.456 & 0.96003 & 2533.571 \\
\hline 0.84697 & 6927.374 & 1.40007 & 4483.431 & 1.22996 & 1950.187 & 0.98806 & 13689.92 & 1.16804 & 2049.107 & 0.97985 & 2590.668 \\
\hline 0.86961 & 7026.692 & 1.41999 & 3411.306 & 1.24014 & 2417.552 & 0.99197 & 13744.19 & 1.17996 & 2021.478 & 1.00013 & 2611.369 \\
\hline 0.89331 & 7498.448 & 1.4399 & 3898.635 & 1.25 & 2865.28 & 0.99601 & 13798.45 & 1.19207 & 1969.387 & 1.01996 & 2687.275 \\
\hline 0.91853 & 7920.546 & 1.46017 & 5433.723 & 1.25986 & 3422.561 & 0.99999 & 13834.63 & 1.20399 & 1918.639 & 1.03977 & 3004.034 \\
\hline 0.94521 & 8144.01 & 1.48008 & 8479.532 & 1.27004 & 3901.027 & 1.00409 & 13852.71 & 1.21591 & 1873.106 & 1.06003 & 3981.705 \\
\hline 0.97328 & 8640.596 & 1.5 & 11379.14 & 1.2799 & 4614.473 & 1.00813 & 13906.98 & 1.22802 & 1859.464 & 1.07983 & 5079.742 \\
\hline 1.00329 & 9236.499 & 1.51992 & 14035.09 & 1.29008 & 5067.977 & 1.0122 & 13961.24 & 1.23995 & 1873.106 & 1.10008 & 7152.804 \\
\hline 1.03497 & 9807.573 & 1.53983 & 16008.77 & 1.29994 & 5913.445 & 1.01641 & 14015.5 & 1.25206 & 1936.756 & 1.11988 & 9450.57 \\
\hline 1.06897 & 10353.82 & 1.5601 & 16544.83 & 1.31011 & 6418.949 & 1.02055 & 14051.68 & 1.26398 & 2034.183 & 1.14014 & 12446.78 \\
\hline 1.10529 & 11520.79 & 1.58001 & 16544.83 & 1.31997 & 7330.607 & 1.02482 & 14069.77 & 1.27609 & 2190.734 & 1.15993 & 16523.9 \\
\hline 1.11709 & 12414.65 & 1.59993 & 15935.67 & 1.33015 & 7941.731 & 1.02903 & 14105.94 & 1.28801 & 2359.332 & 1.17974 & 19592.25 \\
\hline 1.12223 & 12836.75 & 1.61985 & 15667.64 & 1.34001 & 8946.516 & 1.03338 & 14142.12 & 1.29993 & 2575.635 & 1.20001 & 23716.1 \\
\hline 1.12713 & 13209.19 & 1.64011 & 16569.2 & 1.34987 & 9711.3 & 1.03766 & 14178.29 & 1.31204 & 2782.559 & 1.22028 & 27764.14 \\
\hline 1.13236 & 13854.75 & 1.66003 & 18469.79 & 1.36005 & 10854.84 & 1.04197 & 14214.47 & 1.32397 & 3018.691 & 1.24009 & 31185.37 \\
\hline 1.13764 & 14897.58 & 1.67994 & 21393.76 & 1.36991 & 11600.09 & 1.04643 & 14250.65 & 1.33607 & 3281.707 & 1.2599 & 34640.03 \\
\hline 1.14297 & 16039.73 & 1.69986 & 25804.09 & 1.38009 & 12740.13 & 1.05082 & 14286.82 & 1.348 & 3530.578 & 1.28018 & 39281.94 \\
\hline 1.14805 & 17181.87 & 1.72013 & 29069.2 & 1.38995 & 13668.1 & 1.05535 & 14341.09 & 1.35992 & 3758.865 & 1.29999 & 42739.86 \\
\hline 1.15347 & 18671.63 & 1.74004 & 32358.67 & 1.40013 & 14894.57 & 1.05981 & 14377.26 & 1.37203 & 3985.231 & 1.32027 & 47248.33 \\
\hline 1.15895 & 20161.39 & 1.75996 & 36330.41 & 1.40999 & 15948.29 & 1.06442 & 14413.44 & 1.38395 & 4163.908 & 1.34009 & 50999.98 \\
\hline 1.16417 & 22098.08 & 1.77987 & 40692.01 & 1.41985 & 17210.48 & 1.06897 & 14503.88 & 1.39606 & 4377.94 & 1.35991 & 54007.95 \\
\hline 1.16975 & 23885.79 & 1.80014 & 45516.57 & 1.43003 & 18392.09 & 1.07355 & 14558.14 & 1.40798 & 4559.917 & 1.38019 & 56379.78 \\
\hline 1.17539 & 25872.13 & 1.82006 & 52436.65 & 1.43989 & 19654.82 & 1.07828 & 14594.32 & 1.42009 & 4789.301 & 1.40001 & 58018.6 \\
\hline 1.18108 & 28230.91 & 1.83997 & 57577.97 & 1.45006 & 20922.36 & 1.08294 & 14648.58 & 1.43202 & 5157.885 & 1.41983 & 59231.73 \\
\hline 1.1865 & 30366.23 & 1.85989 & 62548.73 & 1.45992 & 22490.19 & 1.08775 & 14702.84 & 1.44394 & 5316.473 & 1.44011 & 61050.55 \\
\hline 1.1923 & 32725.02 & 1.88015 & 66520.47 & 1.4701 & 23661.69 & 1.09249 & 14793.28 & 1.45605 & 5713.682 & 1.45993 & 65479.88 \\
\hline 1.19815 & 34686.53 & 1.90007 & 68835.28 & 1.47996 & 25385.17 & 1.09739 & 14829.46 & 1.46797 & 6244.028 & 1.48022 & 65793.32 \\
\hline 1.20374 & 36846.68 & 1.91999 & 70662.77 & 1.49014 & 26499.64 & 1.10222 & 14883.72 & 1.48008 & 6945.817 & 1.50004 & 68138.25 \\
\hline 1.21573 & 41266.29 & 1.9399 & 71661.79 & 1.5 & 28208.61 & 1.10709 & 14974.16 & 1.492 & 7922.572 & 1.51986 & 71471.13 \\
\hline 1.22182 & 43327.13 & 1.96017 & 72246.59 & 1.50986 & 29562.28 & 1.11212 & 15046.51 & 1.50393 & 8933.475 & 1.54014 & 75927.83 \\
\hline 1.22762 & 45065.18 & 1.98008 & 72831.38 & 1.52004 & 31346.08 & 1.11708 & 15118.86 & 1.51603 & 10211.05 & 1.55995 & 82480.22 \\
\hline 1.23383 & 46778.4 & 2 & 73172.51 & 1.5299 & 32914.53 & 1.12221 & 15209.3 & 1.52796 & 11671.33 & 1.58024 & 86102.46 \\
\hline 1.24608 & 50080.7 & 2.01992 & 73318.71 & 1.54008 & 34900.6 & 1.12726 & 15317.83 & 1.54007 & 13480.48 & 1.60006 & 89171.21 \\
\hline 1.25248 & 51595.28 & 2.03983 & 73732.94 & 1.54994 & 36290.79 & 1.13247 & 15390.18 & 1.55199 & 15832.39 & 1.61988 & 93831.15 \\
\hline 1.25894 & 52638.11 & 2.0601 & 73757.31 & 1.56011 & 38555.82 & 1.13761 & 15480.62 & 1.56391 & 18478.5 & 1.64016 & 97175.36 \\
\hline 1.26547 & 53978.9 & 2.08001 & 73879.14 & 1.56997 & 39935.31 & 1.1428 & 15625.32 & 1.57602 & 21499.4 & 1.65998 & 102253.6 \\
\hline 1.2717 & 55145.87 & 2.09993 & 73879.14 & 1.58015 & 42345.02 & 1.14817 & 15697.67 & 1.58794 & 24625.4 & 1.68026 & 107426 \\
\hline 1.27836 & 55816.26 & 2.11985 & 73757.31 & 1.59001 & 43774.52 & 1.15345 & 15842.38 & 1.60005 & 28000.48 & 1.70008 & 113039.9 \\
\hline 1.29151 & 57405.34 & 2.14011 & 73854.78 & 1.59987 & 45785.66 & 1.15891 & 15950.9 & 1.61198 & 31771.73 & 1.72036 & 120471.7 \\
\hline 1.31905 & 59441.34 & 2.16003 & 73757.31 & 1.61005 & 47331.3 & 1.1643 & 16113.7 & 1.62409 & 36201.8 & 1.74018 & 131285.4 \\
\hline 1.34779 & 60657.98 & 2.17994 & 73879.14 & 1.61991 & 49796.75 & 1.16987 & 16204.13 & 1.63601 & 40821.07 & 1.75999 & 141034.7 \\
\hline 1.37781 & 61104.9 & 2.19986 & 74244.64 & 1.63009 & 51578.44 & 1.17536 & 16366.93 & 1.64793 & 45361.7 & 1.78027 & 150546.4 \\
\hline 1.40919 & 61129.73 & 2.22013 & 74244.64 & 1.63995 & 53737.8 & 1.1809 & 16511.63 & 1.66004 & 50830.27 & 1.80009 & 159425.5 \\
\hline 1.44204 & 61378.03 & 2.24004 & 74512.67 & 1.65013 & 55660.5 & 1.18662 & 16656.33 & 1.67196 & 55316.81 & 1.81991 & 167490 \\
\hline 1.47596 & 61576.66 & 2.25996 & 74610.14 & 1.65999 & 58674.29 & 1.19227 & 16819.12 & 1.68407 & 59201.89 & 1.84019 & 173735.8 \\
\hline 1.51204 & 62271.88 & 2.27987 & 74658.87 & 1.66985 & 60183.04 & 1.19811 & 17000 & 1.69599 & 62964.1 & 1.86002 & 177368.5 \\
\hline 1.54992 & 62892.61 & 2.30014 & 74902.53 & 1.68003 & 63317.93 & 1.20386 & 17198.97 & 1.70792 & 66547.12 & 1.8803 & 185157.9 \\
\hline 1.58975 & 63637.49 & 2.32006 & 75097.47 & 1.68989 & 65073.05 & 1.20982 & 17361.76 & 1.72003 & 69530.74 & 1.90012 & 192062.6 \\
\hline 1.63168 & 64009.93 & 2.33997 & 75097.47 & 1.70006 & 67797.38 & 1.21569 & 17578.81 & 1.73195 & 72876.09 & 1.91994 & 200497.3 \\
\hline 1.67589 & 64456.86 & 2.35989 & 75365.5 & 1.70992 & 70223.11 & 1.22161 & 17832.04 & 1.74406 & 75667.88 & 1.94022 & 210639.4 \\
\hline 1.72255 & 65102.42 & 2.38015 & 75462.96 & 1.7201 & 73020.3 & 1.22774 & 18031.01 & 1.75598 & 78402.71 & 1.96004 & 219889.9 \\
\hline 1.77117 & 65599.01 & 2.40007 & 75487.33 & 1.72996 & 75485.34 & 1.23379 & 18338.5 & 1.76809 & 80476.47 & & \\
\hline 1.82338 & 66045.93 & 2.41999 & 75779.73 & 1.74014 & 78338.99 & 1.24004 & 18664.08 & 1.78001 & 82777.79 & & \\
\hline 1.87875 & 66492.86 & 2.4399 & 75950.29 & 1.75 & 80667.87 & 1.24621 & 18989.66 & 1.79194 & 84524.8 & & \\
\hline 1.9376 & 66939.79 & 2.46017 & 75950.29 & 1.75986 & 82580.73 & 1.25259 & 19242.89 & 1.80405 & 86489.11 & & \\
\hline 2.00025 & 67386.72 & 2.48008 & 76242.69 & 1.77004 & 85535.4 & 1.25888 & 19568.48 & 1.81597 & 87946.32 & & \\
\hline 2.06708 & 67808.81 & 2.5 & 76388.89 & 1.7799 & 87563.68 & 1.26524 & 19912.14 & 1.82808 & 90084.18 & & \\
\hline 2.1375 & 68230.91 & 2.51992 & 76681.29 & 1.79008 & 91229.59 & 1.27182 & 20165.37 & 1.84 & 91410.85 & & \\
\hline 2.214 & 68677.84 & 2.53983 & 76827.49 & 1.79994 & 93758.4 & 1.27831 & 20545.22 & 1.85192 & 93340.05 & & \\
\hline 2.29618 & 69844.82 & 2.5601 & 77119.88 & 1.81011 & 97874.61 & 1.28502 & 20870.8 & 1.86403 & 94912.69 & & \\
\hline 2.38469 & 70614.53 & 2.58001 & 77192.98 & 1.81997 & 100000 & 1.29164 & 21286.82 & 1.87595 & 97017.05 & & \\
\hline 2.4803 & 71707.01 & 2.59993 & 77363.55 & 1.83015 & 102571.4 & 1.2985 & 21594.32 & 1.88806 & 98857.89 & & \\
\hline 2.5839 & 72551.21 & 2.61985 & 77582.85 & 1.84001 & 104594.3 & 1.30526 & 21974.16 & 1.89999 & 101155.3 & & \\
\hline 2.69487 & 73345.75 & 2.64011 & 77534.11 & 1.84987 & 109186.2 & 1.3121 & 22444.44 & 1.91191 & 103074.7 & & \\
\hline 2.81761 & 74636.87 & 2.66003 & 77680.31 & 1.86005 & 111339.6 & 1.31917 & 22788.11 & 1.92402 & 104920.8 & & \\
\hline 2.95207 & 76424.58 & 2.67994 & 78021.44 & 1.86991 & 115774.6 & 1.32616 & 23276.49 & 1.93594 & 106800 & & \\
\hline & & 2.69986 & 77997.08 & 1.88009 & 117597.6 & 1.33338 & 23801.03 & 1.94805 & 108599.5 & & \\
\hline & & 2.72013 & 78240.74 & 1.88995 & 120857.4 & 1.34052 & 24379.84 & 1.95997 & 110544.6 & & \\
\hline
\end{tabular}




\begin{tabular}{|c|c|c|c|c|c|c|c|c|c|c|c|}
\hline & & 2.74004 & 78435.67 & 1.90013 & 124693.5 & 1.3479 & 24832.04 & 1.97208 & 112172.6 & & \\
\hline & & 2.75996 & 78557.5 & 1.90999 & 128400.5 & 1.35519 & 25483.2 & 1.98401 & 114779.4 & & \\
\hline & & 2.77987 & 78849.9 & 1.91985 & 131445.2 & 1.36256 & 26170.54 & 1.99593 & 116105.5 & & \\
\hline & & 2.80014 & 79044.83 & 1.93003 & 135617.4 & 1.37019 & 26731.27 & 2.00804 & 118061.6 & & \\
\hline & & 2.82006 & 79312.87 & 1.93989 & 138022.3 & 1.37773 & 27454.78 & 2.01996 & 119925.5 & & \\
\hline & & 2.83997 & 79239.77 & 1.95006 & 142125.4 & 1.38553 & 28196.38 & 2.03207 & 122201 & & \\
\hline & & 2.85989 & 79654 & 1.95992 & 144928.4 & 1.39323 & 28992.25 & 2.04399 & 124000.7 & & \\
\hline & & 2.88015 & 79629.63 & 1.9701 & 150407.3 & 1.40121 & 29661.5 & 2.05591 & 125958.3 & & \\
\hline & & 2.90007 & 79678.36 & 1.97996 & 153074.4 & 1.40909 & 30511.63 & 2.06802 & 128080.5 & & \\
\hline & & 2.91999 & 79922.03 & 1.99014 & 155788.7 & 1.41706 & 31434.11 & 2.07995 & 130510.7 & & \\
\hline & & 2.9399 & 80116.96 & 2 & 159794.7 & 1.42531 & 32193.8 & 2.09206 & 133126 & & \\
\hline & & 2.96017 & 80287.52 & 2.00986 & 164224.1 & 1.43347 & 33170.54 & 2.10398 & 136362.2 & & \\
\hline & & 2.98008 & 80482.46 & 2.02004 & 168776.2 & 1.44192 & 34183.46 & 2.11609 & 139385.5 & & \\
\hline & & 3 & 80555.56 & 2.0299 & 171433.9 & 1.45026 & 35214.47 & 2.12801 & 141882 & & \\
\hline & & 3.01992 & 80774.85 & 2.04008 & 174473.8 & 1.45891 & 36064.6 & 2.13993 & 144122 & & \\
\hline & & 3.03983 & 81018.52 & 2.04994 & 180011.9 & 1.46746 & 37149.87 & 2.15204 & 147934 & & \\
\hline & & 3.0601 & 81067.25 & 2.06011 & 183562 & 1.4761 & 38253.23 & 2.16397 & 149486.9 & & \\
\hline & & 3.08001 & 81262.18 & 2.06997 & 188650.2 & 1.48506 & 39103.36 & 2.17607 & 154083.1 & & \\
\hline & & 3.09993 & 81408.38 & 2.08015 & 190129.7 & 1.49392 & 40206.72 & 2.188 & 157006.8 & & \\
\hline & & 3.11985 & 81432.75 & 2.09001 & 195018.7 & 1.50309 & 41310.08 & 2.19992 & 158158.7 & & \\
\hline & & 3.14011 & 81725.15 & 2.09987 & 198476.9 & 1.51216 & 42449.61 & 2.21203 & 160823.4 & & \\
\hline & & 3.16003 & 81773.88 & 2.11005 & 204377.3 & 1.52157 & 43317.83 & 2.22395 & 164389.1 & & \\
\hline & & 3.17994 & 81871.35 & 2.11991 & 205578.2 & 1.53086 & 44457.36 & 2.23606 & 168561.1 & & \\
\hline & & 3.19986 & 82139.38 & 2.13009 & 210864.5 & 1.54028 & 45669.25 & 2.24798 & 170330.5 & & \\
\hline & & 3.22013 & 82139.38 & 2.13995 & 214184.9 & 1.55003 & 46664.08 & 2.26009 & 171222.2 & & \\
\hline & & 3.24004 & 81871.35 & 2.15013 & 220121.9 & 1.55968 & 47912.14 & 2.27202 & 172478.4 & & \\
\hline & & 3.25996 & 81920.08 & 2.15999 & 220983.4 & 1.56969 & 49214.47 & 2.28394 & 174107 & & \\
\hline & & 3.27987 & 82236.84 & 2.16985 & 225782.1 & 1.57958 & 50571.06 & 2.29605 & 176486.7 & & \\
\hline & & 3.30014 & 82480.51 & 2.18003 & 231136 & 1.58985 & 51801.03 & 2.30797 & 173381.3 & & \\
\hline & & 3.32006 & 82577.97 & 2.18989 & 233860.4 & 1.6 & 53320.41 & 2.32008 & 175201.3 & & \\
\hline & & 3.33997 & 82748.54 & 2.20006 & 240342.9 & 1.61028 & 54857.88 & 2.332 & 175934.7 & & \\
\hline & & 3.35989 & 83016.57 & 2.20992 & 243651.1 & 1.62095 & 56268.73 & 2.34393 & 175567.6 & & \\
\hline & & 3.38015 & 83016.57 & 2.2201 & 247971.7 & 1.63151 & 57987.08 & 2.35603 & 179272.9 & & \\
\hline & & 3.40007 & 83065.3 & 2.22996 & 254845.2 & 1.64246 & 59741.6 & 2.36796 & 178339.3 & & \\
\hline & & 3.41999 & 83260.23 & 2.24014 & 257346 & 1.6533 & 61423.77 & 2.38007 & 177596 & & \\
\hline & & 3.4399 & 83528.27 & 2.25 & 262934.4 & 1.66454 & 62762.27 & 2.39199 & 177781.5 & & \\
\hline & & 3.46017 & 83674.46 & 2.25986 & 267074.7 & 1.67568 & 64444.44 & 2.40391 & 177225.4 & & \\
\hline & & 3.48008 & 83552.63 & 2.27004 & 273942.3 & 1.68696 & 66072.35 & 2.41602 & 179272.9 & & \\
\hline & & 3.5 & 84088.69 & 2.2799 & 274477.8 & 1.69867 & 67284.24 & 2.42794 & 178153.2 & & \\
\hline & & 3.51992 & 84210.53 & 2.29008 & 280438.2 & 1.71027 & 68731.27 & 2.44005 & 176486.7 & & \\
\hline & & 3.53983 & 84429.82 & 2.29994 & 281535.7 & 1.7223 & 70087.86 & 2.45198 & 175751.1 & & \\
\hline & & 3.5601 & 84405.46 & 2.31011 & 289905.3 & 1.73422 & 71372.09 & 2.46409 & 175384.4 & & \\
\hline & & 3.58001 & 84746.59 & 2.31997 & 294470.3 & 1.7466 & 72222.22 & 2.48793 & 175934.7 & & \\
\hline & & 3.59993 & 84819.69 & 2.33015 & 297359.9 & 1.75887 & 73198.97 & 2.50004 & 178712.2 & & \\
\hline & & 3.61985 & 85209.55 & 2.34001 & 305602.5 & 1.7713 & 74085.27 & & & & \\
\hline & & 3.64011 & 85087.72 & 2.34987 & 309204.6 & 1.78422 & 74682.17 & & & & \\
\hline & & 3.66003 & 85185.19 & 2.36005 & 312238.8 & 1.79701 & 75405.68 & & & & \\
\hline & & 3.67994 & 85526.32 & 2.36991 & 319642.8 & 1.81031 & 76002.58 & & & & \\
\hline & & 3.69986 & 85794.35 & 2.38009 & 325310.9 & 1.82348 & 76509.04 & & & & \\
\hline & & 3.72013 & 86013.65 & 2.38995 & 327862.1 & 1.83717 & 76834.63 & & & & \\
\hline & & 3.74004 & 86038.01 & 2.40013 & 329788.7 & 1.85075 & 77268.73 & & & & \\
\hline & & 3.75996 & 86622.81 & 2.40999 & 336292.8 & 1.86452 & 77594.32 & & & & \\
\hline & & 3.77987 & 86257.31 & 2.41985 & 338930.2 & 1.87884 & 77847.55 & & & & \\
\hline & & 3.80014 & 86232.94 & 2.43003 & 344940.2 & 1.89303 & 78118.86 & & & & \\
\hline & & 3.82006 & 86379.14 & 2.43989 & 349006 & 1.90779 & 78354.01 & & & & \\
\hline & & 3.83997 & 86476.61 & 2.45006 & 357281.9 & 1.92243 & 78607.24 & & & & \\
\hline & & 3.85989 & 86793.37 & 2.45992 & 361493.2 & 1.93765 & 78770.03 & & & & \\
\hline & & 3.88015 & 86963.94 & 2.4701 & 362907.9 & 1.95276 & 79041.34 & & & & \\
\hline & & 3.90007 & 87256.34 & 2.47996 & 368622.5 & 1.9681 & 79222.22 & & & & \\
\hline & & 3.91999 & 87305.07 & 2.49014 & 373696.5 & 1.98405 & 79276.49 & & & & \\
\hline & & 3.9399 & 87646.2 & 2.5 & 378101.3 & 1.99989 & 79385.01 & & & & \\
\hline & & 3.96017 & 87719.3 & 2.50986 & 381811.5 & 2.01637 & 79366.93 & & & & \\
\hline & & & & 2.52004 & 388581.9 & 2.03273 & 79348.84 & & & & \\
\hline & & & & 2.5299 & 391629.4 & 2.04976 & 79330.75 & & & & \\
\hline & & & & 2.54008 & 396245.5 & 2.06667 & 79276.49 & & & & \\
\hline & & & & 2.54994 & 401699.8 & & & & & & \\
\hline & & & & 2.56011 & 408025.2 & & & & & & \\
\hline & & & & 2.56997 & 408822.9 & & & & & & \\
\hline & & & & 2.58015 & 413641.6 & & & & & & \\
\hline & & & & 2.59001 & 420155.1 & & & & & & \\
\hline & & & & 2.59987 & 424278 & & & & & & \\
\hline & & & & 2.61005 & 426771.2 & & & & & & \\
\hline & & & & 2.61991 & 434338.8 & & & & & & \\
\hline & & & & 2.63009 & 438600.9 & & & & & & \\
\hline & & & & 2.63995 & 442040.7 & & & & & & \\
\hline & & & & 2.65013 & 446378.4 & & & & & & \\
\hline & & & & 2.65999 & 451639.8 & & & & & & \\
\hline & & & & 2.66985 & 456963.2 & & & & & & \\
\hline & & & & 2.68003 & 461447.3 & & & & & & \\
\hline & & & & 2.68989 & 463253.3 & & & & & & \\
\hline & & & & 2.70006 & 471467.8 & & & & & & \\
\hline & & & & 2.70992 & 475165.4 & & & & & & \\
\hline
\end{tabular}




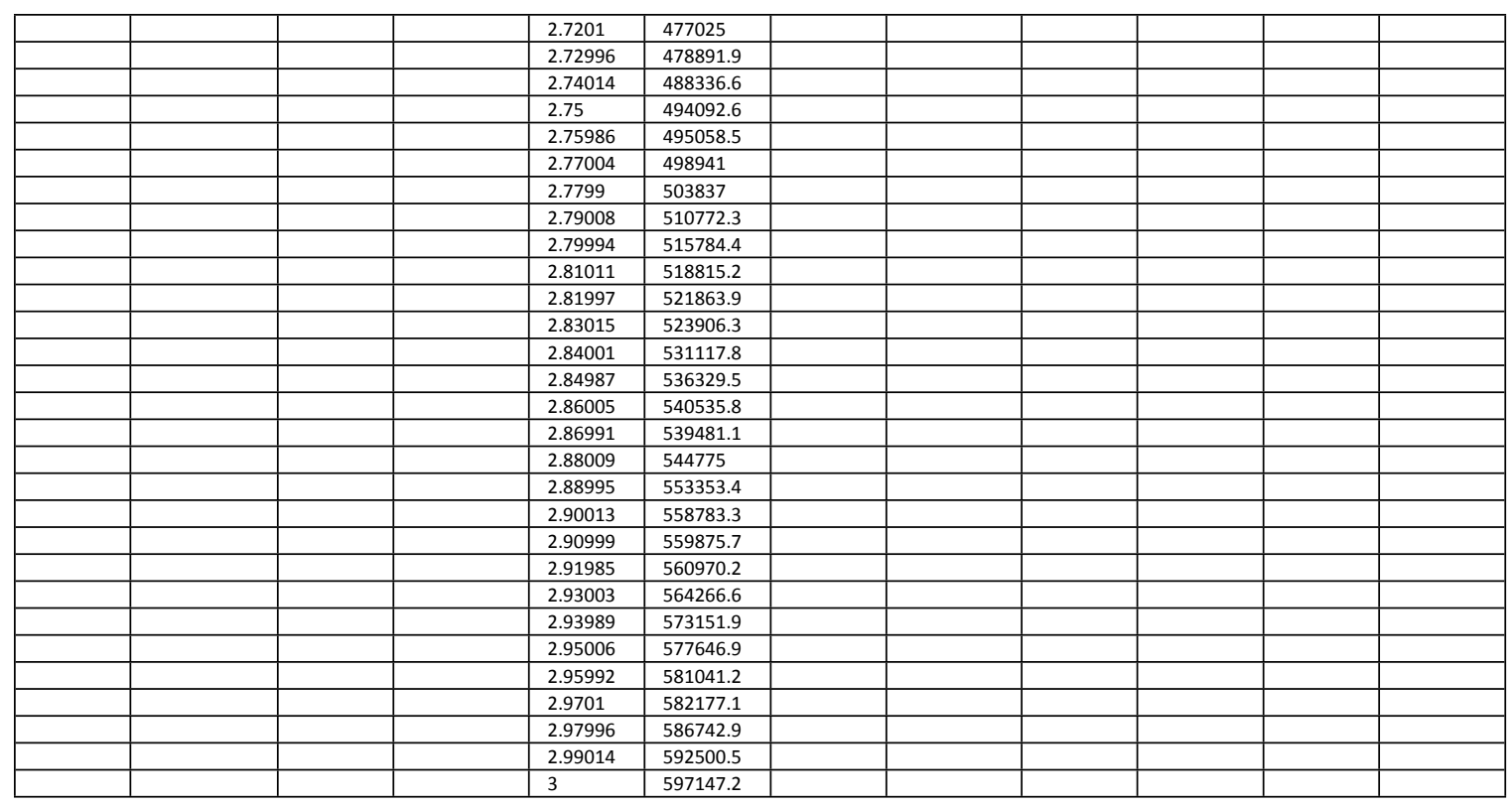

Table S13 continued.

\begin{tabular}{|c|c|c|c|c|c|}
\hline \multicolumn{2}{|c|}{$\mathrm{Cu}_{2} \mathrm{SnS}_{3}$} & \multicolumn{2}{|c|}{$\mathrm{Cu}_{2} \mathrm{GeS}_{3}$} & \multicolumn{2}{|c|}{$\mathrm{AgBiS}_{2}$} \\
\hline $\mathrm{eV}$ & $\alpha\left(\mathrm{cm}^{-1}\right)$ & $\mathrm{eV}$ & $\alpha\left(\mathrm{cm}^{-1}\right)$ & $\mathrm{eV}$ & $\alpha\left(\mathrm{cm}^{-1}\right)$ \\
\hline 0.96017 & 2.05933 & 0.90999 & 9424.501 & 1.15668 & 46.0713 \\
\hline 0.96017 & 3.49129 & 0.91998 & 9515.67 & 1.16431 & 406.905 \\
\hline 0.96017 & 5.89847 & 0.92998 & 9578.348 & 1.17203 & 573.2714 \\
\hline 0.96017 & 9.93078 & 0.93997 & 9652.422 & 1.17986 & 751.5258 \\
\hline 0.96017 & 16.83621 & 0.94996 & 9698.006 & 1.1878 & 932.814 \\
\hline 0.96017 & 28.54337 & 0.95995 & 9766.382 & 1.19569 & 1269.287 \\
\hline 0.97973 & 50.45053 & 0.96994 & 9800.57 & 1.20384 & 1548.317 \\
\hline 0.97973 & 116.9158 & 0.97993 & 9840.456 & 1.22048 & 2024.718 \\
\hline 0.97973 & 186.8501 & 0.98993 & 9880.342 & 1.22897 & 2361.821 \\
\hline 0.97973 & 208.8136 & 1.00008 & 9908.832 & 1.23758 & 2721.045 \\
\hline 0.97973 & 270.0055 & 1.01007 & 9954.416 & 1.24615 & 3253.911 \\
\hline 0.97973 & 301.7436 & 1.02007 & 9988.604 & 1.255 & 3693.361 \\
\hline 0.97973 & 349.1294 & 1.03006 & 10022.79 & 1.26399 & 4192.161 \\
\hline 0.97973 & 387.4675 & 1.04005 & 10062.68 & 1.2731 & 4664.705 \\
\hline 0.97973 & 418.2325 & 1.05004 & 10096.87 & 1.28234 & 5038.084 \\
\hline 1 & 1000 & 1.06003 & 10148.15 & 1.29172 & 5661.957 \\
\hline 1.02027 & 1701.254 & 1.07002 & 10176.64 & 1.30124 & 6300.177 \\
\hline 1.03983 & 2382.725 & 1.08002 & 10239.32 & 1.31071 & 6975.598 \\
\hline 1.0601 & 3325.602 & 1.09001 & 10296.3 & 1.32051 & 7898.032 \\
\hline 1.07966 & 4153.376 & 1.1 & 10341.88 & 1.33046 & 8509.055 \\
\hline 1.09993 & 4923.883 & 1.10999 & 10381.77 & 1.34056 & 9682.249 \\
\hline 1.1202 & 5638.08 & 1.11998 & 10421.65 & 1.35081 & 11072.07 \\
\hline 1.13976 & 6730.608 & 1.12998 & 10467.24 & 1.36122 & 11810.71 \\
\hline 1.16003 & 7547.933 & 1.13997 & 10501.42 & 1.37159 & 12724.44 \\
\hline 1.18029 & 8702.984 & 1.14996 & 10547.01 & 1.38233 & 13983.99 \\
\hline 1.19986 & 9692.266 & 1.15995 & 10569.8 & 1.39324 & 14954 \\
\hline 1.22013 & 10794 & 1.16994 & 10609.69 & 1.40431 & 16110.9 \\
\hline 1.23969 & 11855.13 & 1.17993 & 10655.27 & 1.41557 & 17793.84 \\
\hline 1.25996 & 12885.63 & 1.18993 & 10689.46 & 1.42701 & 19122.88 \\
\hline 1.28022 & 14300.61 & 1.19992 & 10712.25 & 1.43841 & 20449.35 \\
\hline 1.29979 & 15489.78 & 1.21007 & 10752.14 & 1.45022 & 22031.4 \\
\hline 1.32006 & 16489.01 & 1.22007 & 10780.63 & 1.46223 & 23559.62 \\
\hline 1.34032 & 17798.24 & 1.23006 & 10826.21 & 1.47443 & 25006.81 \\
\hline 1.35989 & 18946.39 & 1.24005 & 10871.79 & 1.48685 & 26741.42 \\
\hline 1.38015 & 20098.67 & 1.25004 & 10905.98 & 1.49947 & 28596.35 \\
\hline 1.39972 & 22074.53 & 1.26003 & 10934.47 & 1.51231 & 30656.01 \\
\hline 1.41999 & 23094 & 1.27002 & 10968.66 & 1.52512 & 32701.16 \\
\hline 1.44025 & 24076.8 & 1.28002 & 11025.64 & 1.53841 & 33357.47 \\
\hline 1.45982 & 25101.42 & 1.29001 & 11065.53 & 1.55193 & 32377.86 \\
\hline 1.48008 & 26169.65 & 1.3 & 11111.11 & 1.56569 & 33274.72 \\
\hline 1.50035 & 27283.33 & 1.30999 & 11145.3 & 1.57969 & 34969.49 \\
\hline 1.51992 & 28345.8 & 1.31998 & 11207.98 & 1.59395 & 37025.47 \\
\hline
\end{tabular}




\begin{tabular}{|c|c|c|c|c|c|}
\hline 1.54018 & 28942.66 & 1.32998 & 11282.05 & 1.60819 & 39202.32 \\
\hline 1.55975 & 29861.6 & 1.33997 & 11327.64 & 1.62296 & 40791.69 \\
\hline 1.58001 & 30702.91 & 1.34996 & 11384.62 & 1.63802 & 42762.98 \\
\hline 1.60028 & 31024.47 & 1.35995 & 11481.48 & 1.65335 & 44718.32 \\
\hline 1.61985 & 31898.54 & 1.36994 & 11572.65 & 1.66898 & 46763.06 \\
\hline 1.64011 & 32797.23 & 1.37993 & 11646.72 & 1.6849 & 48658.97 \\
\hline 1.65968 & 33025.83 & 1.38993 & 11732.19 & 1.70082 & 51137.31 \\
\hline 1.67994 & 34311.91 & 1.40008 & 11834.76 & 1.71736 & 53475.56 \\
\hline 1.70021 & 34912.94 & 1.41007 & 11988.6 & 1.73422 & 55920.73 \\
\hline 1.71978 & 36021.44 & 1.42007 & 12170.94 & 1.75142 & 58332.63 \\
\hline 1.74004 & 37165.14 & 1.43006 & 12433.05 & 1.76896 & 60999.89 \\
\hline 1.76031 & 37816.14 & 1.44005 & 12638.18 & 1.78686 & 63001.77 \\
\hline 1.77987 & 39152.56 & 1.45004 & 12854.7 & 1.80512 & 65719.08 \\
\hline 1.80014 & 40960.76 & 1.46003 & 13071.23 & 1.82341 & 68383.52 \\
\hline 1.81971 & 42852.47 & 1.47002 & 13316.24 & 1.84243 & 71510.35 \\
\hline 1.83997 & 44521.23 & 1.48002 & 13566.95 & 1.86185 & 74594.65 \\
\hline 1.86024 & 46577.37 & 1.49001 & 13931.62 & 1.88169 & 77618.93 \\
\hline 1.8798 & 48223.41 & 1.5 & 14353.28 & 1.90195 & 80765.82 \\
\hline 1.90007 & 50275.62 & 1.50999 & 14860.4 & 1.92266 & 84879.46 \\
\hline 1.92034 & 51871.9 & 1.51998 & 15492.88 & 1.94341 & 87665.02 \\
\hline 1.9399 & 53333.3 & 1.52998 & 16131.05 & 1.96504 & 91673.5 \\
\hline 1.96017 & 56773.78 & 1.53997 & 16951.57 & 1.98715 & 95627.44 \\
\hline 1.97973 & 58576.37 & 1.54996 & 18108.26 & 2.00976 & 100000 \\
\hline 2 & 60436.2 & 1.55995 & 19470.09 & 2.03289 & 104572.5 \\
\hline 2.02027 & 62355.07 & 1.56994 & 20643.87 & 2.05656 & 108812.2 \\
\hline 2.03983 & 65461.8 & 1.57993 & 21675.21 & 2.08033 & 113223.7 \\
\hline 2.0601 & 68485.05 & 1.58993 & 22245.01 & 2.10513 & 117521.9 \\
\hline 2.07966 & 70905.31 & 1.59992 & 22490.03 & 2.13052 & 122590.7 \\
\hline 2.09993 & 74438.03 & 1.61007 & 22564.1 & 2.15654 & 128196.1 \\
\hline 2.1202 & 76005.43 & 1.62007 & 22598.29 & 2.1832 & 133725.3 \\
\hline 2.13976 & 78418.64 & 1.63006 & 22655.27 & 2.21052 & 139839.9 \\
\hline 2.16003 & 80069.86 & 1.64005 & 22774.93 & 2.238 & 146234.1 \\
\hline 2.18029 & 83767.76 & 1.65004 & 22917.38 & 2.26673 & 152920.6 \\
\hline 2.19986 & 87941.34 & 1.66003 & 23116.81 & 2.2962 & 159516.2 \\
\hline 2.22013 & 88247.29 & 1.67002 & 23287.75 & 2.32644 & 165160.9 \\
\hline 2.23969 & 90105.46 & 1.68002 & 23538.46 & 2.3575 & 172284.4 \\
\hline 2.25996 & 92966.35 & 1.69001 & 23817.66 & 2.38939 & 179715.1 \\
\hline 2.28022 & 95585.53 & 1.7 & 24062.68 & 2.42216 & 187466.4 \\
\hline 2.29979 & 98963.52 & 1.70999 & 24267.81 & 2.4552 & 196038.3 \\
\hline 2.32006 & 101047.3 & 1.71998 & 24461.54 & 2.48981 & 204493.5 \\
\hline 2.34032 & 102460.9 & 1.72998 & 24592.59 & 2.52541 & 213313.5 \\
\hline 2.35989 & 103894.2 & 1.73997 & 24700.85 & 2.56205 & 222513.9 \\
\hline 2.38015 & 106450.9 & 1.74996 & 24735.04 & 2.59976 & 229816.3 \\
\hline 2.39972 & 108692.4 & 1.75995 & 24774.93 & 2.6386 & 239728.4 \\
\hline 2.41999 & 112143.4 & 1.76994 & 24746.44 & 2.67786 & 248828.9 \\
\hline 2.44025 & 115704 & 1.77993 & 24769.23 & 2.71908 & 258917.1 \\
\hline 2.45982 & 115704 & 1.78993 & 24803.42 & 2.7616 & 268746 \\
\hline 2.48008 & 118963.8 & 1.79992 & 24797.72 & 2.80547 & 278255.9 \\
\hline 2.50035 & 121891.3 & 1.81007 & 24786.32 & 2.85075 & 288102.4 \\
\hline 2.51992 & 122740.9 & 1.82007 & 24786.32 & 2.89752 & 299782.9 \\
\hline 2.54018 & 126638 & 1.83006 & 24797.72 & 2.94493 & 308086.8 \\
\hline 2.55975 & 128409.5 & 1.84005 & 24752.14 & 2.99486 & 318197.6 \\
\hline 2.58001 & 130205.8 & 1.85004 & 24723.65 & 3.04653 & 328640.1 \\
\hline 2.60028 & 131569.5 & 1.86003 & 24700.85 & & \\
\hline 2.61985 & 136693.1 & 1.87002 & 24678.06 & & \\
\hline 2.64011 & 138605.3 & 1.88002 & 24678.06 & & \\
\hline 2.65968 & 138605.3 & 1.89001 & 24689.46 & & \\
\hline 2.67994 & 138605.3 & 1.9 & 24706.55 & & \\
\hline 2.70021 & 141033.2 & 1.90999 & 24706.55 & & \\
\hline 2.71978 & 143503.6 & 1.91998 & 24695.16 & & \\
\hline 2.74004 & 144503.8 & 1.92998 & 24695.16 & & \\
\hline 2.76031 & 146017.3 & 1.93997 & 24700.85 & & \\
\hline 2.77987 & 149091.9 & 1.94996 & 24717.95 & & \\
\hline 2.80014 & 148575 & 1.95995 & 24717.95 & & \\
\hline 2.81971 & 150131.1 & 1.96994 & 24695.16 & & \\
\hline 2.83997 & 151177.5 & 1.97993 & 24695.16 & & \\
\hline 2.86024 & 152760.9 & 1.98993 & 24678.06 & & \\
\hline 2.8798 & 154897.8 & 2.00008 & 24678.06 & & \\
\hline 2.90007 & 154360.8 & 2.01007 & 24678.06 & & \\
\hline 2.92034 & 153825.6 & 2.02007 & 24666.67 & & \\
\hline 2.9399 & 150653.4 & 2.03006 & 24643.87 & & \\
\hline \multirow[t]{6}{*}{2.96017} & 162051.5 & 2.04005 & 24672.36 & & \\
\hline & & 2.05004 & 24666.67 & & \\
\hline & & 2.06003 & 24649.57 & & \\
\hline & & 2.07002 & 24638.18 & & \\
\hline & & 2.08002 & 24603.99 & & \\
\hline & & 2.09001 & 24569.8 & & \\
\hline
\end{tabular}


Table S13 continued.

\begin{tabular}{|c|c|c|c|c|c|c|c|c|c|}
\hline \multicolumn{2}{|l|}{ CZTS } & \multicolumn{2}{|c|}{ CZTSe } & \multicolumn{2}{|l|}{ CCTS } & \multicolumn{2}{|c|}{ CZGSe } & \multicolumn{2}{|c|}{ AZTSe } \\
\hline $\mathrm{eV}$ & $\alpha\left(\mathrm{cm}^{-1}\right)$ & $\mathrm{eV}$ & $\alpha\left(\mathrm{cm}^{-1}\right)$ & $\mathrm{eV}$ & $\alpha\left(\mathrm{cm}^{-1}\right)$ & $\mathrm{eV}$ & $\alpha\left(\mathrm{cm}^{-1}\right)$ & $\mathrm{eV}$ & $\alpha\left(\mathrm{cm}^{-1}\right)$ \\
\hline 1.03997 & 110.1988 & 0.74005 & 303.1326 & 1.00996 & 23366.88 & 1.32005 & 169.1281 & 1.30993 & 50952.38 \\
\hline 1.06011 & 308.8844 & 0.75987 & 661.3147 & 1.02008 & 23103.13 & 1.32005 & 214.9865 & 1.32006 & 50952.38 \\
\hline 1.07994 & 607.6779 & 0.78002 & 1165.914 & 1.03004 & 22852.34 & 1.34006 & 682.3778 & 1.33003 & 51111.11 \\
\hline 1.10008 & 981.3789 & 0.80016 & 1802.114 & 1.04 & 22693.26 & 1.34997 & 991.5433 & 1.34 & 51305.11 \\
\hline 1.1199 & 1536.012 & 0.81998 & 2496.197 & 1.04996 & 22554.97 & 1.36006 & 1565.163 & 1.34998 & 51675.49 \\
\hline 1.14005 & 2120.951 & 0.84013 & 3319.62 & 1.06008 & 22417.53 & 1.36998 & 2342.91 & 1.35995 & 52469.14 \\
\hline 1.15987 & 2883.128 & 0.85995 & 4428.519 & 1.07004 & 22368.65 & 1.38006 & 2940.483 & 1.36992 & 53968.25 \\
\hline 1.18002 & 3715.934 & 0.8801 & 5428.675 & 1.08 & 22290.66 & 1.38998 & 3817.991 & 1.38005 & 55908.29 \\
\hline 1.20016 & 4819.402 & 0.89992 & 6571.841 & 1.08996 & 22232.34 & 1.40007 & 5373.911 & 1.39003 & 58218.69 \\
\hline 1.21998 & 5797.828 & 0.92006 & 7881.314 & 1.10008 & 22290.66 & 1.40998 & 6436.764 & 1.4 & 60194 \\
\hline 1.24013 & 7062.844 & 0.92006 & 7264.814 & 1.11004 & 22290.66 & 1.42007 & 7841.902 & 1.40997 & 61622.57 \\
\hline 1.25995 & 8364.673 & 0.93989 & 9217.77 & 1.12 & 22437.11 & 1.42999 & 9079.15 & 1.41995 & 62768.96 \\
\hline 1.2801 & 9906.457 & 0.96003 & 10780.85 & 1.12996 & 22417.53 & 1.44007 & 10691.67 & 1.43008 & 63562.61 \\
\hline 1.29992 & 11406.25 & 0.97986 & 12067.93 & 1.14008 & 22614.13 & 1.44999 & 11763.58 & 1.44005 & 64320.99 \\
\hline 1.32006 & 13679 & 1.02014 & 15360.12 & 1.15004 & 22742.85 & 1.46008 & 12833.49 & 1.45002 & 65008.82 \\
\hline 1.33989 & 15121.39 & 1.03997 & 16926.67 & 1.16 & 22872.3 & 1.46999 & 14120.13 & 1.46 & 66031.75 \\
\hline 1.36003 & 16926.67 & 1.06011 & 18420.7 & 1.16996 & 23133.41 & 1.48008 & 15080.76 & 1.46997 & 67266.31 \\
\hline 1.37986 & 18829.12 & 1.07994 & 20046.6 & 1.18008 & 23417.94 & 1.50008 & 16912.81 & 1.47994 & 68377.43 \\
\hline 1.4 & 20945.39 & 1.10008 & 21816.02 & 1.19004 & 23747.41 & 1.51 & 18101.83 & 1.49007 & 70123.46 \\
\hline 1.42014 & 22937.4 & 1.14005 & 24420.53 & 1.2 & 24071 & 1.53992 & 20474.03 & 1.50005 & 71940.04 \\
\hline 1.43997 & 24806.06 & 1.15987 & 26576.01 & 1.20996 & 24430.98 & 1.55001 & 20869.02 & 1.51002 & 73738.98 \\
\hline 1.46011 & 26659.4 & 1.20016 & 28921.74 & 1.22008 & 24818 & 1.55993 & 21959.99 & 1.51999 & 75555.56 \\
\hline 1.47994 & 28831.28 & 1.24013 & 31672.35 & 1.23004 & 25233.18 & 1.57001 & 22863.97 & 1.52997 & 77019.4 \\
\hline 1.50008 & 30791.82 & 1.2801 & 34252.63 & 1.24 & 25532.39 & 1.57993 & 22863.97 & 1.53994 & 78024.69 \\
\hline 1.5199 & 32172.37 & 1.29992 & 36239.62 & 1.24996 & 26038.97 & 1.59002 & 23855.77 & 1.55007 & 78888.89 \\
\hline 1.54005 & 33826.08 & 1.32006 & 37745.98 & 1.26008 & 26567.2 & 1.59993 & 24732.54 & 1.56004 & 79488.54 \\
\hline 1.55987 & 35453.56 & 1.33989 & 38462.19 & 1.27004 & 27047.05 & 1.61002 & 25750.66 & 1.57002 & 80123.46 \\
\hline 1.58002 & 37864.42 & 1.36003 & 40566.1 & 1.28 & 27619.84 & 1.61994 & 26753.83 & 1.57999 & 80987.65 \\
\hline 1.60016 & 39438.32 & 1.37986 & 41726.14 & 1.28996 & 28180.14 & 1.63002 & 27796.07 & 1.58996 & 81746.03 \\
\hline 1.61998 & 40949.15 & 1.4 & 44146.67 & 1.30008 & 28953.36 & 1.63994 & 28878.92 & 1.59994 & 82627.87 \\
\hline 1.64013 & 42384.87 & 1.42014 & 45267.06 & 1.31004 & 29657.01 & 1.65003 & 29436.06 & 1.61007 & 83527.34 \\
\hline 1.65995 & 43189.1 & 1.43997 & 45837.88 & 1.32 & 30404.31 & 1.67995 & 33012.03 & 1.62004 & 84409.17 \\
\hline 1.6801 & 45125.47 & 1.46011 & 48043.28 & 1.32996 & 31088.89 & 1.69995 & 35634.22 & 1.63001 & 85396.83 \\
\hline 1.69992 & 46707.62 & 1.47994 & 49417.13 & 1.34008 & 31900.11 & 1.71004 & 35938.14 & 1.63999 & 86313.93 \\
\hline 1.72006 & 48345.24 & 1.50008 & 50830.27 & 1.35004 & 32604.13 & 1.71995 & 37022.41 & 1.64996 & 87266.31 \\
\hline 1.73989 & 48954.87 & 1.5199 & 53109.24 & 1.36 & 33338.25 & 1.73004 & 38464.69 & 1.65993 & 88342.15 \\
\hline 1.76003 & 50040.28 & 1.54005 & 53947.68 & 1.36996 & 34208.16 & 1.73996 & 39123.61 & 1.67006 & 89523.81 \\
\hline 1.77986 & 51632.74 & 1.55987 & 56543.27 & 1.38008 & 35285.15 & 1.75004 & 40303.99 & 1.68004 & 90564.37 \\
\hline 1.8 & 52612.44 & 1.58002 & 58525.74 & 1.39004 & 36190.06 & 1.75996 & 41963.11 & 1.69001 & 91710.76 \\
\hline 1.82014 & 54286.75 & 1.60016 & 59823.35 & 1.4 & 37150.6 & 1.77005 & 42321.01 & 1.69998 & 92892.42 \\
\hline 1.83997 & 54971.31 & 1.61998 & 61920.83 & 1.40996 & 38053.48 & 1.77996 & 43321.05 & 1.70996 & 93968.25 \\
\hline 1.86011 & 56014.35 & 1.64013 & 63691.52 & 1.42008 & 39165.94 & 1.79005 & 44533.42 & 1.71993 & 95167.55 \\
\hline 1.87994 & 56366.41 & 1.65995 & 64900.03 & 1.43004 & 40469.64 & 1.79997 & 45392.57 & 1.73006 & 96349.21 \\
\hline 1.90008 & 57796.93 & 1.6801 & 67809.81 & 1.44 & 41543.77 & 1.81005 & 46072.24 & 1.74003 & 97530.86 \\
\hline 1.9199 & 58525.74 & 1.69992 & 69967.76 & 1.44996 & 42572 & 1.81997 & 47160.92 & 1.75001 & 98765.43 \\
\hline 1.94005 & 60199.35 & 1.72006 & 72420.9 & 1.46008 & 43931.48 & 1.83006 & 48172.94 & 1.75998 & 99964.73 \\
\hline 1.95987 & 61341.6 & 1.73989 & 76622.05 & 1.47004 & 45196.05 & 1.83997 & 49102.31 & 1.76995 & 100917.1 \\
\hline 1.98002 & 63691.52 & 1.76003 & 78566.62 & 1.48 & 46153.19 & 1.85006 & 50049.6 & 1.77993 & 101816.6 \\
\hline 2.00016 & 64697.03 & 1.77986 & 82864.28 & 1.48996 & 47378.17 & 1.85998 & 50049.6 & 1.79006 & 102627.9 \\
\hline 2.01998 & 66965.38 & 1.8 & 84967.28 & 1.50008 & 48784.53 & 1.87006 & 52442.87 & 1.80003 & 103368.6 \\
\hline 2.04013 & 68236.01 & 1.82014 & 89334.75 & 1.51004 & 49795.92 & 1.87998 & 52666.04 & 1.81 & 104197.5 \\
\hline 2.05995 & 69748.91 & 1.83997 & 91029.82 & 1.52 & 50917.12 & 1.89007 & 53682.09 & 1.81998 & 104620.8 \\
\hline 2.0801 & 73334.13 & 1.86011 & 93926.71 & 1.52996 & 52040.84 & 1.89998 & 54255.01 & 1.82995 & 105432.1 \\
\hline 2.09992 & 74960.06 & 1.87994 & 100313.8 & 1.54008 & 53468.76 & 1.91007 & 56129.76 & 1.83993 & 105996.5 \\
\hline 2.12006 & 76862.46 & 1.90008 & 102537.9 & 1.55004 & 54434.48 & 1.91999 & 57700.59 & 1.85005 & 106860.7 \\
\hline 2.13989 & 76622.05 & 1.9199 & 106466 & 1.56 & 55587.27 & 1.93007 & 58564.56 & 1.86003 & 107301.6 \\
\hline 2.16003 & 78813.14 & 1.94005 & 109510.5 & 1.56996 & 56714.93 & 1.93999 & 58316.4 & 1.87 & 107777.8 \\
\hline 2.17986 & 81321.26 & 1.95987 & 114420.4 & 1.58008 & 57865.46 & 1.95008 & 59189.58 & 1.87998 & 108412.7 \\
\hline 2.2 & 82864.28 & 1.98002 & 118432.1 & 1.59004 & 59013.56 & 1.95999 & 60846.05 & 1.88995 & 108959.4 \\
\hline 2.22014 & 84967.28 & 2.00016 & 121818.8 & 1.6 & 60158.18 & 1.97008 & 61626.13 & 1.90008 & 109523.8 \\
\hline 2.23997 & 87123.65 & 2.01998 & 126485.5 & 1.60996 & 61217.98 & 1.98 & 62151.73 & 1.91005 & 110000 \\
\hline 2.26011 & 89896.23 & 2.04013 & 129695.6 & 1.62008 & 62214.92 & 1.99008 & 63891.09 & 1.92002 & 110634.9 \\
\hline 2.27994 & 92466.92 & 2.05995 & 133404.4 & 1.63004 & 63255.71 & 2 & 64162.97 & 1.93 & 110952.4 \\
\hline 2.30008 & 96612.65 & 2.0801 & 140261.6 & 1.64 & 64201.69 & 2.00992 & 66239.3 & 1.93997 & 111305.1 \\
\hline 2.3199 & 98137.89 & 2.09992 & 144272.5 & 1.64996 & 65332.73 & 2.02 & 66380.09 & 1.94995 & 111869.5 \\
\hline 2.34005 & 99687.21 & 2.12006 & 147010 & 1.66008 & 66454.68 & 2.02992 & 67374.01 & 1.96007 & 112186.9 \\
\hline 2.35987 & 101897.4 & 2.13989 & 153601.2 & 1.67004 & 67184 & 2.04001 & 68966.04 & 1.97005 & 112539.7 \\
\hline 2.38002 & 106133 & 2.16003 & 157993.6 & 1.68 & 67862.03 & 2.04992 & 69406.73 & 1.98002 & 113015.9 \\
\hline 2.40016 & 109168 & 2.17986 & 161496.6 & 1.68996 & 68997.29 & 2.06001 & 70896.11 & 1.99 & 113298.1 \\
\hline 2.41998 & 110891.4 & 2.2 & 167158.8 & 1.70008 & 69998.56 & 2.06993 & 71957.65 & 1.99997 & 113844.8 \\
\hline 2.44013 & 118432.1 & 2.22014 & 171938.9 & 1.71004 & 70674.14 & 2.08001 & 73035.09 & 2.00994 & 114250.4 \\
\hline 2.45995 & 108826.5 & 2.23997 & 170865 & 1.72 & 71262.84 & 2.08993 & 74128.66 & 2.02007 & 114567.9 \\
\hline 2.4801 & 112642.1 & 2.26011 & 175751.1 & 1.72996 & 72076.38 & 2.10002 & 75238.61 & 2.03005 & 114832.5 \\
\hline 2.49992 & 118061.6 & 2.27994 & 183630.8 & 1.74008 & 72867.38 & 2.10993 & 75079.03 & 2.04002 & 115202.8 \\
\hline
\end{tabular}




\begin{tabular}{|c|c|c|c|c|c|c|c|c|c|}
\hline 2.52006 & 120679.3 & 2.30008 & 185946.4 & 1.75004 & 73570.65 & 2.12002 & 76527.48 & 2.04999 & 115626.1 \\
\hline 2.53989 & 121818.8 & 2.3199 & 188882 & 1.76 & 74215.87 & 2.12994 & 76041.59 & 2.05997 & 115555.6 \\
\hline 2.56003 & 121818.8 & 2.34005 & 196733.2 & 1.76996 & 74703.49 & 2.14002 & 77673.35 & 2.06994 & 115996.5 \\
\hline 2.62014 & 147934 & 2.35987 & 200466 & 1.78008 & 75523.33 & 2.14994 & 78335.81 & 2.08007 & 116349.2 \\
\hline 2.63997 & 151688.4 & 2.38002 & 202359 & 1.79004 & 75787.58 & 2.16003 & 78335.81 & 2.09004 & 116596.1 \\
\hline 2.66011 & 152641.8 & 2.40016 & 202359 & 1.8 & 76686.25 & 2.16994 & 80016.8 & 2.10002 & 116631.4 \\
\hline 2.67994 & 157006.8 & 2.41998 & 208145.7 & 1.80996 & 76988.16 & 2.18003 & 81214.91 & 2.10999 & 116754.9 \\
\hline 2.75987 & 161496.6 & 2.44013 & 208145.7 & 1.82008 & 77561.7 & 2.18995 & 82256.13 & 2.11996 & 117495.6 \\
\hline 2.78002 & 166114.7 & 2.45995 & 214097.8 & 1.83004 & 77765.14 & 2.20003 & 83487.77 & 2.12994 & 118060 \\
\hline 2.80016 & 170865 & 2.4801 & 220220.2 & 1.84 & 78241.92 & 2.20995 & 84737.85 & 2.14007 & 118589.1 \\
\hline 2.81998 & 170865 & 2.49992 & 220220.2 & 1.84996 & 78893.66 & 2.22004 & 84917.96 & 2.15004 & 119047.6 \\
\hline 2.84013 & 174107 & 2.52006 & 220220.2 & 1.86008 & 79308.07 & 2.22995 & 87109.3 & 2.16001 & 119329.8 \\
\hline 2.85995 & 178525.7 & 2.53989 & 220220.2 & 1.87004 & 79829.16 & 2.24004 & 88413.61 & 2.16999 & 119488.5 \\
\hline 2.8801 & 180211.4 & 2.53989 & 199839 & 1.88 & 79794.31 & 2.24996 & 88413.61 & 2.17996 & 119400.4 \\
\hline 2.89992 & 175751.1 & 2.56003 & 214097.8 & 1.88996 & 80494.11 & 2.26004 & 90119.32 & 2.18993 & 119400.4 \\
\hline 2.92006 & 185946.4 & 2.57986 & 218844.7 & 1.90008 & 80846.31 & 2.26996 & 91857.93 & & \\
\hline 2.97986 & 177410.6 & 2.6 & 220220.2 & 1.91004 & 80987.62 & 2.28005 & 92641.37 & & \\
\hline 3 & 184785 & 2.62014 & 224398.7 & 1.92 & 81377.5 & 2.28996 & 94028.51 & & \\
\hline 3.02014 & 189474.6 & 2.63997 & 225809.1 & 1.92996 & 81484.16 & 2.30005 & 96250.39 & & \\
\hline 3.06011 & 188882 & 2.66011 & 225102.8 & 1.94008 & 82127.04 & 2.30997 & 98734.17 & & \\
\hline 3.07994 & 191263.7 & 2.67994 & 225809.1 & 1.95004 & 82306.51 & 2.32005 & 99154.33 & & \\
\hline 3.10008 & 191263.7 & 2.70008 & 225809.1 & 1.96 & 83064.6 & 2.32997 & 100852.9 & & \\
\hline \multirow[t]{16}{*}{3.14005} & 202359 & 2.7199 & 225809.1 & 1.96996 & 83355.23 & 2.34006 & 100852.9 & & \\
\hline & & 2.74005 & 223696.8 & 1.98008 & 83282.48 & 2.34997 & 102580.5 & & \\
\hline & & 2.75987 & 224398.7 & 1.99004 & 83866.29 & 2.36006 & 104337.8 & & \\
\hline & & 2.78002 & 230093.7 & & & 2.36998 & 103895.7 & & \\
\hline & & 2.80016 & 230093.7 & & & 2.38006 & 105675.5 & & \\
\hline & & 2.81998 & 232266.4 & & & 2.38998 & 108172.6 & & \\
\hline & & 2.84013 & 232266.4 & & & & & & \\
\hline & & 2.85995 & 231539.9 & & & & & & \\
\hline & & 2.8801 & 228656.6 & & & & & & \\
\hline & & 2.89992 & 228656.6 & & & & & & \\
\hline & & 2.92006 & 223696.8 & & & & & & \\
\hline & & 2.93989 & 222299.6 & & & & & & \\
\hline & & 2.96003 & 222299.6 & & & & & & \\
\hline & & 2.97986 & 223696.8 & & & & & & \\
\hline & & 3 & 226517.6 & & & & & & \\
\hline & & 3.02014 & 239657.9 & & & & & & \\
\hline
\end{tabular}

1. Choi, Y. C.; Lee, D. U.; Noh, J. H.; Kim, E. K.; Seok, S. I., Highly Improved Sb2S3SensitizedInorganic-Organic Heterojunction Solar Cells and Quantification of Traps by Deep-Level Transient Spectroscopy. Advanced Functional Materials 2014, 24 (23), 3587-3592.

2. Tang, R.; Wang, X.; Lian, W.; Huang, J.; Wei, Q.; Huang, M.; Yin, Y.; Jiang, C.; Yang, S.; Xing, G.; Chen, S.; Zhu, C.; Hao, X.; Green, M. A.; Chen, T., Hydrothermal deposition of antimony selenosulfide thin films enables solar cells with $10 \%$ efficiency. Nature Energy 2020, 5 (8), 587-595.

3. Li, Z.; Liang, X.; Li, G.; Liu, H.; Zhang, H.; Guo, J.; Chen, J.; Shen, K.; San, X.; Yu, W.; Schropp, R. E. I.; Mai, Y., 9.2\%-efficient core-shell structured antimony selenide nanorod array solar cells. Nat Commun 2019, $10(1), 125$.

4. Yun, H. S.; Park, B. W.; Choi, Y. C.; Im, J.; Shin, T. J.; Seok, S. I., Efficient Nanostructured TiO2/SnS Heterojunction Solar Cells. Advanced Energy Materials 2019, 1901343.

5. Liu, S. C.; Dai, C. M.; Min, Y.; Hou, Y.; Proppe, A. H.; Zhou, Y.; Chen, C.; Chen, S.; Tang, J.; Xue, D. J.; Sargent, E. H.; Hu, J. S., An antibonding valence band maximum enables defect-tolerant and stable GeSe photovoltaics. Nature Communications 2021, 12 (1), 670.

6. Banu, S.; Ahn, S. J.; Ahn, S. K.; Yoon, K.; Cho, A., Fabrication and characterization of cost-efficient CuSbS2 thin film solar cells using hybrid inks. Solar Energy Materials and Solar Cells 2016, 151, 14-23.

7. Welch, A. W.; Baranowski, L. L.; Peng, H.; Hempel, H.; Eichberger, R.; Unold, T.; Lany, S.; Wolden, C.; Zakutayev, A., Trade-Offs in Thin Film Solar Cells with Layered Chalcostibite Photovoltaic Absorbers. Advanced Energy Materials 2017, 7 (11), 1601935.

8. Chantana, J.; Tai, K.; Hayashi, H.; Nishimura, T.; Kawano, Y.; Minemoto, T., Investigation of carrier recombination of $\mathrm{Na}$-doped $\mathrm{Cu}_{2} \mathrm{SnS}_{3}$ solar cell for its improved conversion efficiency of $5.1 \%$. Solar Energy Materials and Solar Cells 2020, 206, 110261.

9. Umehara, M.; Tajima, S.; Aoki, Y.; Takeda, Y.; Motohiro, T., Cu2Sn1-xGexS3solar cells fabricated with a graded bandgap structure. Applied Physics Express 2016, 9 (7), 072301. 
10. Jin, X.; Zhang, L.; Jiang, G.; Liu, W.; Zhu, C., High open-circuit voltage of ternary Cu2GeS3 thin film solar cells from combustion synthesized Cu-Ge alloy. Solar Energy Materials and Solar Cells 2017, 160, 319-327.

11. Bernechea, M.; Miller, N. C.; Xercavins, G.; So, D.; Stavrinadis, A.; Konstantatos, G., Solutionprocessed solar cells based on environmentally friendly AgBiS2 nanocrystals. Nature Photonics 2016, 10 (8), 521-525.

12. Yan, C.; Huang, J.; Sun, K.; Johnston, S.; Zhang, Y.; Sun, H.; Pu, A.; He, M.; Liu, F.; Eder, K.; Yang, L.; Cairney, J. M.; Ekins-Daukes, N. J.; Hameiri, Z.; Stride, J. A.; Chen, S.; Green, M. A.; Hao, X., $\mathrm{Cu}_{2} \mathrm{ZnSnS}_{4}$ solar cells with over $10 \%$ power conversion efficiency enabled by heterojunction heat treatment. Nature Energy 2018.

13. Wang, W.; Winkler, M. T.; Gunawan, O.; Gokmen, T.; Todorov, T. K.; Zhu, Y.; Mitzi, D. B., Device Characteristics of CZTSSe Thin-Film Solar Cells with 12.6\% Efficiency. Advanced Energy Materials 2014, 4 (7), 1301465.

14. Son, D.-H.; Kim, S.-H.; Kim, S.-Y.; Kim, Y.-I.; Sim, J.-H.; Park, S.-N.; Jeon, D.-H.; Hwang, D.-K.; Sung, S.-J.; Kang, J.-K.; Yang, K.-J.; Kim, D.-H., Effect of solid- $\mathrm{H}_{2} \mathrm{~S}$ gas reactions on CZTSSe thin film growth and photovoltaic properties of a 12.62\% efficiency device. Journal of Materials Chemistry A 2019.

15. Li, J.; Huang, Y.; Huang, J.; Liang, G.; Zhang, Y.; Rey, G.; Guo, F.; Su, Z.; Zhu, H.; Cai, L.; Sun, K.;

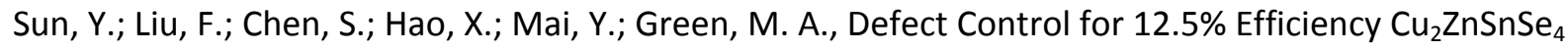
Kesterite Thin-Film Solar Cells by Engineering of Local Chemical Environment. Adv Mater 2020, e2005268.

16. Shin, D.; Zhu, T.; Huang, X.; Gunawan, O.; Blum, V.; Mitzi, D. B., Earth-Abundant Chalcogenide Photovoltaic Devices with over $5 \%$ Efficiency Based on a $\mathrm{Cu}_{2} \mathrm{BaSn}(\mathrm{S}, \mathrm{Se})_{4}$ Absorber. Advanced Materials 2017, 29 (24), 1606945.

17. Su, Z.; Liang, G.; Fan, P.; Luo, J.; Zheng, Z.; Xie, Z.; Wang, W.; Chen, S.; Hu, J.; Wei, Y.; Yan, C.; Huang, J.; Hao, X.; Liu, F., Device Postannealing Enabling over 12\% Efficient Solution-Processed $\mathrm{Cu}_{2} \mathrm{ZnSnS}_{4}$ Solar Cells with Cd ${ }^{2+}$ Substitution. Advanced Materials 2020, 32 (32), 2000121.

18. Hadke, S.; Levcenko, S.; Sai Gautam, G.; Hages, C. J.; Márquez, J. A.; Izquierdo-Roca, V.; Carter, E. A.; Unold, T.; Wong, L. H., Suppressed Deep Traps and Bandgap Fluctuations in $\mathrm{Cu}_{2} \mathrm{CdSnS}_{4}$ Solar Cells with $\approx 8 \%$ Efficiency. Advanced Energy Materials 2019, 9 (45), 1902509.

19. Gershon, T.; Sardashti, K.; Gunawan, O.; Mankad, R.; Singh, S.; Lee, Y. S.; Ott, J. A.; Kummel, A.; Haight, R., Photovoltaic Device with over $5 \%$ Efficiency Based on an n-Type $\mathrm{Ag}_{2} \mathrm{ZnSnSe}{ }_{4}$ Absorber. Advanced Energy Materials 2016, 6 (22), 1601182.

20. Choubrac, L.; Bär, M.; Kozina, X.; Félix, R.; Wilks, R. G.; Brammertz, G.; Levcenko, S.; Arzel, L.; Barreau, N.; Harel, S.; Meuris, M.; Vermang, B., Sn substitution by Ge: Strategies to overcome the open circuit voltage deficit of kesterite solar cells. ACS Applied Energy Materials 2020, 3 (6), 5830-5839. 